\title{
A vorticity-based fully-mixed formulation for the 3D Brinkman-Darcy problem
}

\author{
Mario Alvarez ${ }^{\mathrm{a}, \mathrm{b}}$, Gabriel N. Gatica ${ }^{\mathrm{b}, \mathrm{c}}$, Ricardo Ruiz-Baier*,d \\ ${ }^{a}$ Sección de Matemática, Sede Occidente, Universidad de Costa Rica, San Ramón de Alajuela, Costa Rica. \\ ${ }^{b} C I^{2} M A$, Universidad de Concepción, Casilla 160-C, Concepción, Chile. \\ ${ }^{c}$ Departamento de Ingeniería Matemática, Universidad de Concepción, Casilla 160-C, Concepción, Chile. \\ ${ }^{d}$ Mathematical Institute, University of Oxford, A. Wiles Building, Radcliffe Observatory Quarter, Woodstock Road, Oxford \\ OX2 6GG, United Kingdom.
}

\begin{abstract}
We propose and analyze a fully-mixed finite element method to numerically approximate the flow patterns of a viscous fluid within a highly permeable medium (an array of low concentration fixed particles), described by Brinkman equations, and its interaction with non-viscous flow within classical porous media governed by Darcy's law. The system is formulated in terms of velocity and pressure of the porous medium, together with vorticity, velocity and pressure of the fluid. In addition, and for sake of the analysis, the tangential component of the vorticity is supposed to vanish on the whole boundary of the fluid, whereas null normal components of both velocities are assumed on the respective boundaries, except on the interface where suitable transmission conditions are considered. In this way, the derivation of the corresponding mixed variational formulation leads to a Lagrange multiplier enforcing the pressure continuity across the interface, whereas mass balance results from essential boundary conditions on each domain. As a consequence, a typical saddle-point operator equation is obtained, and hence the classical Babuška-Brezzi theory is applied to establish the well-posedness of the continuous and discrete schemes. In particular, we remark that the continuous and discrete inf-sup conditions of the main bilinear form are proved by using suitably chosen injective operators to get lower bounds of the corresponding suprema, which constitutes a previously known technique, recently denominated $T$-coercivity. In turn, and consistently with the above, the stability of the Galerkin scheme requires that the curl of the finite element subspace approximating the vorticity be contained in the space where the discrete velocity of the fluid lives, which yields Raviart-Thomas and Nédélec finite element subspaces as feasible choices. Then we show that the aforementioned constraint can be avoided by augmenting the mixed formulation with a residual arising from the Brinkman momentum equation. Finally, several numerical examples illustrating the satisfactory performance of the methods and confirming the theoretical rates of convergence are reported.
\end{abstract}

Key words: Brinkman equation, Darcy equation, vorticity-based formulation, mixed finite elements, error analysis, viscous and non-viscous flows, augmented methods

2000 MSC: 65N30, 65N12, 76D07, 65N15

\footnotetext{
*Corresponding author: Phone: +441865 615168.

Email addresses: mguadamuz@ci2ma.udec.cl (Mario Alvarez), ggatica@ci2ma.udec.cl (Gabriel N. Gatica), ruizbaier@maths.ox.ac.uk (Ricardo Ruiz-Baier)

Funding: This work was partially supported by CONICYT-Chile through BASAL project CMM, Universidad de Chile, and project Anillo ACT1118 (ANANUM); by the Ministery of Education through the project REDOC.CTA of the Graduate School, Universidad de Concepción; by Centro de Investigación en Ingeniería Matemática $\left(\mathrm{CI}^{2} \mathrm{MA}\right)$, Universidad de Concepción, and by the Elsevier Mathematical Sciences Sponsorship Fund.
} 


\section{Introduction}

This paper is motivated by the numerical approximation of flow patterns in an heterogeneous media composed by a porous medium, where Darcy equations govern the flow behavior of a non-viscous incompressible fluid, and a much more permeable domain, where the laminar flow exhibits viscous effects and can be described by the linear Brinkman model. The two domains are separated by an essentially fixed interface, across which the flow passes from viscous to a non-viscous regime. According to the discussion in [7], we can assume that the Brinkman domain consists of an array of low concentration fixed particles, whereas the Darcy domain is a classical porous medium constituted by connected porous matrices. Such a scenario is often encountered in e.g. the modelling of surface and subsurface flow in porous media, petroleum reservoirs, or perfusion of physiological fluids into soft tissues, focusing typically on filtration or other similar processes of interest. We are also interested in accurately recovering the additional vorticity field (vectorial for three-dimensional flows and perpendicular to the plane of the flow and therefore considered scalar in 2D), which yields better information on circulation effects of the free fluid, sometimes observed near interfaces.

At the interface of the two domains, and depending on the specific form of the problem at hand, one typically requires preservation of physical quantities such as normal velocities, normal stresses, and so on. An abundant body of literature is devoted to different ways of treating the interface conditions, from both mathematical and numerical perspectives. These basically include sequential sub-structuring methods, where decoupled subproblems are solved on each subdomain, followed by an updating of the interface field values, then using these values as boundary data to solve a local problem on the other subdomain, and iterating in some adequate manner (see e.g. [8, 16, 29, 32]); and monolithic, fully coupled approaches where all sought fields are computed at once, for instance by a single operator acting on the two media or with the aid of Lagrange multipliers specifically designed to impose continuity of fields to be conserved across the interface (see for instance $[6,14,18,19]$ ). Our method follows the latter strategy. Up to our knowledge, the coupling of Brinkman and Darcy flows has been only addressed in terms of the primal unknowns of velocity and pressure $[12,18,29]$. Vorticity-based formulations for the Stokes-Darcy coupling were introduced in [10] and later studied in [9, 17]. Differences with respect to these contributions include a slightly different formulation (we do not assume that the fluid boundary coincides with the interface between both domains, which is used in $[10,9]$ ); the analysis itself differs in that here we set pressure continuity across the interface using a Lagrange multiplier, and the normal stress conditions are weakly imposed. In addition, the proposed treatment does not require higher regularity of the fluid pressure as in e.g. [9]. At the discrete level, that work involves a family of nonconforming discretizations consisting in Nédélec elements for vorticity, piecewise constant elements for velocity, and Crouzeix-Raviart elements for the pressure. In contrast, here we use a finite element family where the curl of the subspace approximating the vorticity must be contained in the space where the discrete velocity of the fluid lives, and hence Raviart-Thomas and Nédélec finite elements for velocities and vorticity, respectively, become feasible choices. In turn, the pressures and the Lagrange multiplier are approximated, respectively, by discontinuous and continuous piecewise polynomials. Finally, our numerical tests also include the 3D case.

A general advantage of formulations involving vorticity is that this additional field can be accessed directly, without postprocessing; and it is straightforward to include non-inertial effects by modifying initial and boundary data $[4,5]$. For instance, for external flows it is known that boundary conditions are better suited for vorticity than for pressure. Moreover, in many flow regimes the vorticity is concentrated in a specific region of the domain, which suggests the use of vorticity as guide to mesh refinement.

The contents of the paper are organized as follows. In the remainder of the present section we recall basic terminology and some properties of functional spaces, and introduce further standard notations. In Section 2 we describe the coupled problem of interest and derive a first version of its mixed variational formulation. The solvability analysis of the later is carried out in Section 3. We first identify the non-trivial solutions of the associated homogeneous problem, and then reformulate the original continuous formulation in order to be able to guarantee unique solvability of it. The classical Babuška-Brezzi is then applied in such a way that the continuous inf-sup conditions of the main bilinear form are established by employing a known approach that has been recently referred as $T$-coercivity. Then, in Section 4 we introduce the associated Galerkin scheme and adapt the arguments from the continuous case to prove that, under suitable assumptions on the finite element subspaces involved, it is well-posed. Next, in Section 5 we modify the mixed formulation by incorporating a residual arising from the Brinkman momentum equation, and show 
that the resulting augmented scheme, yielding a strongly elliptic main bilinear form, does not require the aforementioned constraint. Finally, several numerical examples illustrating the good performance of the mixed finite element methods and confirming the theoretical rates of convergence are provided in Section 6 .

We end this section by specifying some notations to be employed throughout the paper. In particular, we utilize standard simplified terminology for Sobolev spaces and norms. For instance, if $\mathcal{O} \subseteq \mathbb{R}^{3}$ is a domain, $\mathcal{S} \subseteq \mathbb{R}^{3}$ is a Lipschitz surface, and $r \in \mathbb{R}$, we define

$$
\mathbf{H}^{r}(\mathcal{O}):=\left[\mathrm{H}^{r}(\mathcal{O})\right]^{3} \text { and } \mathbf{H}^{r}(\mathcal{S}):=\left[\mathrm{H}^{r}(\mathcal{S})\right]^{3}
$$

However, when $r=0$ we usually write $\mathbf{L}^{2}(\mathcal{O})$ and $\mathbf{L}^{2}(\mathcal{S})$ instead of $\mathbf{H}^{0}(\mathcal{O})$ and $\mathbf{H}^{0}(\mathcal{S})$, respectively. The corresponding norms are denoted by $\|\cdot\|_{r, \mathcal{O}}\left(\right.$ for $\mathrm{H}^{r}(\mathcal{O})$ and $\mathbf{H}^{r}(\mathcal{O})$ ) and $\|\cdot\|_{r, \mathcal{S}}\left(\right.$ for $\mathrm{H}^{r}(\mathcal{S})$ and $\left.\mathbf{H}^{r}(\mathcal{S})\right)$. In general, given any Hilbert space $\mathrm{H}$, we use $\mathbf{H}$ to denote $\mathrm{H}^{3}$. In turn, in the realm of mixed methods (see [13]) one usually needs the Hilbert spaces

$$
\mathbf{H}(\operatorname{div} ; \mathcal{O}):=\left\{\boldsymbol{v} \in \mathbf{L}^{2}(\mathcal{O}): \quad \operatorname{div} \boldsymbol{v} \in \mathrm{L}^{2}(\mathcal{O})\right\}, \quad \mathbf{H}(\operatorname{curl} ; \mathcal{O}):=\left\{\boldsymbol{v} \in \mathbf{L}^{2}(\mathcal{O}): \quad \operatorname{curl} \boldsymbol{v} \in \mathbf{L}^{2}(\mathcal{O})\right\}
$$

normed, respectively, with

$$
\|\boldsymbol{v}\|_{\operatorname{div} ; \mathcal{O}}:=\left\{\|\boldsymbol{v}\|_{0, \mathcal{O}}^{2}+\|\operatorname{div} \boldsymbol{v}\|_{0, \mathcal{O}}^{2}\right\}^{1 / 2}, \quad\|\boldsymbol{v}\|_{\text {curl } ; \mathcal{O}}:=\left\{\|\boldsymbol{v}\|_{0, \mathcal{O}}^{2}+\|\operatorname{curl} \boldsymbol{v}\|_{0, \mathcal{O}}^{2}\right\}^{1 / 2}
$$

where, for any vector field $\boldsymbol{v}:=\left(v_{1}, v_{2}, v_{3}\right)^{\mathrm{t}} \in \mathbf{L}^{2}(\mathcal{O})$,

$$
\operatorname{div} \boldsymbol{v}:=\sum_{i=1}^{3} \partial_{i} v_{i} \quad \text { and } \quad \operatorname{curl} \boldsymbol{v}:=\nabla \times \boldsymbol{v}=\left(\begin{array}{l}
\partial_{2} v_{3}-\partial_{3} v_{2} \\
\partial_{3} v_{1}-\partial_{1} v_{3} \\
\partial_{1} v_{2}-\partial_{2} v_{1}
\end{array}\right)
$$

In addition, we also recall the orthogonal decomposition

$$
\mathrm{L}^{2}(\mathcal{O})=\mathrm{L}_{0}^{2}(\mathcal{O}) \oplus P_{0}(\mathcal{O})
$$

where $P_{0}(\mathcal{O})$ is the space of constant functions on $\mathcal{O}$, and

$$
\mathrm{L}_{0}^{2}(\mathcal{O})=P_{0}(\mathcal{O})^{\perp}:=\left\{q \in \mathrm{L}^{2}(\mathcal{O}): \quad \int_{\mathcal{O}} q=0\right\}
$$

Equivalently, each $q \in \mathrm{L}^{2}(\mathcal{O})$ can be uniquely decomposed as $q=q_{0}+c$, with

$$
q_{0}:=q-\frac{1}{|\mathcal{O}|} \int_{\mathcal{O}} q \in \mathrm{L}_{0}^{2}(\mathcal{O}) \quad \text { and } \quad c:=\frac{1}{|\mathcal{O}|} \int_{\mathcal{O}} q \in \mathbb{R} .
$$

Certainly, $\mathrm{L}_{0}^{2}(\mathcal{O})$ is endowed with the usual norm of $\mathrm{L}^{2}(\mathcal{O})$, and it is easy to see that there holds

$$
\|q\|_{0, \mathcal{O}}^{2}=\left\|q_{0}\right\|_{0, \mathcal{O}}^{2}+|\mathcal{O}| c^{2}
$$

Finally, in what follows $\mathbf{0}$ stands for a generic null vector (including the null functional and operator), and we use $C$ and $c$, with or without subscripts, bars, tildes or hats, to denote generic constants independent of the discretization parameters, which may take different values in different occurrences.

\section{The coupled problem and its mixed formulation}

We first let $\Omega_{\mathrm{B}}$ and $\Omega_{\mathrm{D}}$ be bounded and simply connected polyhedral Lipschitz domains in $\mathbb{R}^{3}$ such that $\partial \Omega_{\mathrm{B}} \cap \partial \Omega_{\mathrm{D}}=: \Sigma \neq \emptyset$ and $\Omega_{\mathrm{B}} \cap \Omega_{\mathrm{D}}=\emptyset$, and set $\Omega:=\bar{\Omega}_{B} \cup \bar{\Omega}_{D}$ with boundary $\Gamma=\partial \Omega$ split into $\Gamma_{\mathrm{B}}$ and $\Gamma_{\mathrm{D}}$ (see the sketch in Figure 2.1). Note that the interface $\Sigma$ between $\Omega_{\mathrm{B}}$ and $\Omega_{\mathrm{D}}$ does not necessarily coincide with $\partial \Omega_{\mathrm{B}}$ (as it was assumed in e.g. $[9,17]$ ). Then, given source terms $\boldsymbol{f}_{\mathrm{D}} \in \mathbf{L}^{2}\left(\Omega_{\mathrm{D}}\right)$ and $\boldsymbol{f}_{\mathrm{B}} \in \mathbf{L}^{2}\left(\Omega_{\mathrm{B}}\right)$, we are interested in the Brinkman-Darcy coupled problem, which is formulated in what follows in terms of the fluid velocity $\boldsymbol{u}_{\mathrm{B}}$, the fluid pressure $p_{\mathrm{B}}$, the fluid vorticity $\boldsymbol{\omega}_{\mathrm{B}}$, the Darcy velocity $\boldsymbol{u}_{\mathrm{D}}$, and the Darcy 


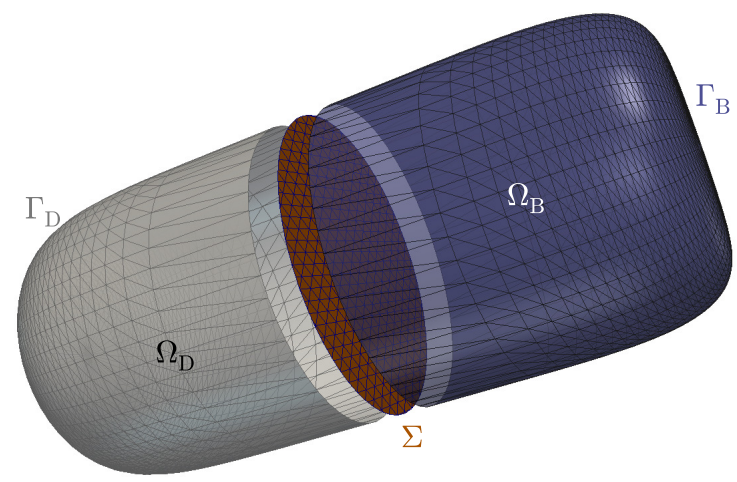

Figure 2.1: Sketch of the domains occupied by the incompressible fluid and by the porous medium ( $\Omega_{\mathrm{B}}$ and $\Omega_{\mathrm{D}}$, respectively), interface $\Sigma$, and corresponding boundaries.

pressure $p_{\mathrm{D}}$. More precisely, the sets of equations in the Brinkman and Darcy domains $\Omega_{\mathrm{B}}$ and $\Omega_{\mathrm{D}}$, are given, respectively, by

$$
\left.\begin{array}{rl}
\alpha \boldsymbol{u}_{\mathrm{B}}+\nu \operatorname{curl} \boldsymbol{\omega}_{\mathrm{B}}+\nabla p_{\mathrm{B}} & =\boldsymbol{f}_{\mathrm{B}} \\
\boldsymbol{\omega}_{\mathrm{B}}-\operatorname{curl} \boldsymbol{u}_{\mathrm{B}} & =\mathbf{0} \\
\operatorname{div} \boldsymbol{u}_{\mathrm{B}} & =0
\end{array}\right\} \quad \text { in } \Omega_{\mathrm{B}}
$$

and

$$
\left.\begin{array}{rl}
\mu \boldsymbol{u}_{\mathrm{D}}+\nabla p_{\mathrm{D}} & =\boldsymbol{f}_{\mathrm{D}} \\
\operatorname{div} \boldsymbol{u}_{\mathrm{D}} & =0
\end{array}\right\} \quad \text { in } \quad \Omega_{\mathrm{D}}
$$

where $\nu>0$ is the kinematic viscosity of the fluid, $\mu>0$ depends on this viscosity and on the permeability of the porous medium, which is assumed to be homogeneous, and $\alpha>0$ is a parameter related to the relaxation time (typically proportional to the inverse of the timestep after a Rothe type time discretization). In turn, the corresponding transmission conditions become

$$
\boldsymbol{u}_{\mathrm{D}} \cdot \boldsymbol{n}=\boldsymbol{u}_{\mathrm{B}} \cdot \boldsymbol{n} \quad \text { and } \quad p_{\mathrm{D}}=p_{\mathrm{B}} \quad \text { on } \Sigma,
$$

where $\boldsymbol{n}$ stands for the outward normal at $\Omega_{\mathrm{B}}$ and $\Omega_{\mathrm{D}}$, whereas the boundary conditions reduce to

$$
\boldsymbol{\omega}_{\mathrm{B}} \times \boldsymbol{n}=0 \quad \text { on } \quad \partial \Omega_{\mathrm{B}}=\Sigma \cup \Gamma_{\mathrm{B}}, \quad \boldsymbol{u}_{\mathrm{B}} \cdot \boldsymbol{n}=0 \quad \text { on } \quad \Gamma_{\mathrm{B}}, \quad \text { and } \quad \boldsymbol{u}_{\mathrm{D}} \cdot \boldsymbol{n}=0 \quad \text { on } \quad \Gamma_{\mathrm{D}} .
$$

Evidently, this choice of boundary data (especially those applied on the vorticity) is driven mainly by easiness of the subsequent analysis, but we stress that other conditions could be incorporated without compromising the main ideas in this work.

We now aim to derive the mixed variational formulation of (2.1) - (2.4). We begin by testing the first equation in (2.1) with functions in the space

$$
\mathbf{H}_{\mathrm{B}}\left(\operatorname{div} ; \Omega_{\mathrm{B}}\right):=\left\{\boldsymbol{v}_{\mathrm{B}} \in \mathbf{H}\left(\operatorname{div} ; \Omega_{\mathrm{B}}\right): \quad \boldsymbol{v}_{\mathrm{B}} \cdot \boldsymbol{n}=0 \quad \text { on } \quad \Gamma_{\mathrm{B}}\right\} .
$$

To this end, we need to recall that the fact that $\boldsymbol{v}_{\mathrm{B}} \cdot \boldsymbol{n}=0$ on $\Gamma_{\mathrm{B}}$ guarantees that $\left.\boldsymbol{v}_{\mathrm{B}} \cdot \boldsymbol{n}\right|_{\Sigma}$ belongs to $\mathrm{H}^{-1 / 2}(\Sigma)$ for each $\boldsymbol{v}_{\mathrm{B}} \in \mathbf{H}_{\mathrm{B}}\left(\right.$ div; $\left.\Omega_{\mathrm{B}}\right)$ (see the beginning of Section 3 below for further details on this issue). In this way, integrating by parts and using the respective boundary conditions, we find that

$$
\alpha \int_{\Omega_{\mathrm{B}}} \boldsymbol{u}_{\mathrm{B}} \cdot \boldsymbol{v}_{\mathrm{B}}+\nu \int_{\Omega_{\mathrm{B}}} \boldsymbol{v}_{\mathrm{B}} \cdot \operatorname{curl} \boldsymbol{\omega}_{\mathrm{B}}-\int_{\Omega_{\mathrm{B}}} p_{\mathrm{B}} \operatorname{div} \boldsymbol{v}_{\mathrm{B}}+\left\langle\boldsymbol{v}_{\mathrm{B}} \cdot \boldsymbol{n}, \lambda\right\rangle_{\Sigma}=\int_{\Omega_{\mathrm{B}}} \boldsymbol{f}_{\mathrm{B}} \cdot \boldsymbol{v}_{\mathrm{B}} \quad \forall \boldsymbol{v}_{\mathrm{B}} \in \mathbf{H}_{\mathrm{B}}\left(\operatorname{div} ; \Omega_{\mathrm{B}}\right),
$$

where, thanks to the second transmission condition in (2.3), we have introduced the auxiliary unknown

$$
\lambda:=\left.p_{\mathrm{D}}\right|_{\Sigma}=\left.p_{\mathrm{B}}\right|_{\Sigma} \in \mathrm{H}^{1 / 2}(\Sigma),
$$


and $\langle\cdot, \cdot\rangle_{\Sigma}$ denotes the duality pairing of $\mathrm{H}^{-1 / 2}(\Sigma)$ and $\mathrm{H}^{1 / 2}(\Sigma)$ with respect to the $\mathrm{L}^{2}(\Sigma)$-inner product. Furthermore, it will become clear below that $\lambda$ can also be seen as the Lagrange multiplier enforcing the continuity of pressure across the interface $\Sigma$. Next, we define

$$
\mathbf{H}_{0}\left(\operatorname{curl} ; \Omega_{\mathrm{B}}\right):=\left\{\boldsymbol{z}_{\mathrm{B}} \in \mathbf{H}\left(\operatorname{curl} ; \Omega_{\mathrm{B}}\right): \quad \boldsymbol{z}_{\mathrm{B}} \times \boldsymbol{n}=\mathbf{0} \quad \text { on } \quad \partial \Omega_{\mathrm{B}}\right\},
$$

so that testing the second equation in (2.1) with functions in this space, and integrating by parts, we obtain

$$
\int_{\Omega_{\mathrm{B}}} \boldsymbol{\omega}_{\mathrm{B}} \cdot \boldsymbol{z}_{\mathrm{B}}-\int_{\Omega_{\mathrm{B}}} \boldsymbol{u}_{\mathrm{B}} \cdot \operatorname{curl} \boldsymbol{z}_{\mathrm{B}}=0 \quad \forall \boldsymbol{z}_{\mathrm{B}} \in \mathbf{H}_{0}\left(\operatorname{curl} ; \Omega_{\mathrm{B}}\right) .
$$

In turn, the third equation in (2.1) is initially tested as

$$
\int_{\Omega_{\mathrm{B}}} q_{\mathrm{B}} \operatorname{div} \boldsymbol{u}_{\mathrm{B}}=0 \quad \forall q_{\mathrm{B}} \in \mathrm{L}^{2}\left(\Omega_{\mathrm{B}}\right) .
$$

On the other hand, in order to deal with the equations in the Darcy domain, we now set

$$
\mathbf{H}_{\mathrm{D}}\left(\operatorname{div} ; \Omega_{\mathrm{D}}\right):=\left\{\boldsymbol{v}_{\mathrm{D}} \in \mathbf{H}\left(\operatorname{div} ; \Omega_{\mathrm{D}}\right): \quad \boldsymbol{v}_{\mathrm{D}} \cdot \boldsymbol{n}=0 \quad \text { on } \quad \Gamma_{\mathrm{D}}\right\},
$$

and test the first equation of (2.2) with functions in this space. Thus, integrating by parts, using the corresponding boundary conditions, noting that the normal $\boldsymbol{n}$ on $\Sigma$ points inward $\Omega_{\mathrm{D}}$, and recalling that $\lambda:=\left.p_{\mathrm{D}}\right|_{\Sigma}$, we get

$$
\mu \int_{\Omega_{\mathrm{D}}} \boldsymbol{u}_{\mathrm{D}} \cdot \boldsymbol{v}_{\mathrm{D}}-\int_{\Omega_{\mathrm{D}}} p_{\mathrm{D}} \operatorname{div} \boldsymbol{v}_{\mathrm{D}}-\left\langle\boldsymbol{v}_{\mathrm{D}} \cdot \boldsymbol{n}, \lambda\right\rangle_{\Sigma}=\int_{\Omega_{\mathrm{D}}} \boldsymbol{f}_{\mathrm{D}} \cdot \boldsymbol{v}_{\mathrm{D}} \quad \forall \boldsymbol{v}_{\mathrm{D}} \in \mathbf{H}_{\mathrm{D}}\left(\operatorname{div} ; \Omega_{\mathrm{D}}\right) .
$$

In addition, similarly as for the incompressibility condition in $\Omega_{\mathrm{B}}$, the second equation in (2.2) is initially tested as

$$
\int_{\Omega_{\mathrm{D}}} q_{\mathrm{D}} \operatorname{div} \boldsymbol{v}_{\mathrm{D}}=0 \quad \forall q_{\mathrm{D}} \in \mathrm{L}^{2}\left(\Omega_{\mathrm{D}}\right) .
$$

We end the present derivation with the weak imposition of the essential transmission condition given by the first equation in (2.3), that is

$$
\left\langle\boldsymbol{u}_{\mathrm{B}} \cdot \boldsymbol{n}-\boldsymbol{u}_{\mathrm{D}} \cdot \boldsymbol{n}, \xi\right\rangle_{\Sigma}=0 \quad \forall \xi \in \mathrm{H}^{1 / 2}(\Sigma) .
$$

Consequently, reordering (2.5) - (2.10) in a suitable way, namely placing each set of equations $\{(2.5)$, (2.6), (2.8) $\}$ and $\{(2.7),(2.9),(2.10)\}$ into a single equation each, we arrive at the mixed formulation of $(2.1)$ - (2.4): Find $\overrightarrow{\boldsymbol{u}}:=\left(\boldsymbol{u}_{\mathrm{B}}, \boldsymbol{\omega}_{\mathrm{B}}, \boldsymbol{u}_{\mathrm{D}}\right) \in \mathbf{H}$ and $\vec{p}:=\left(p_{\mathrm{B}}, p_{\mathrm{D}}, \lambda\right) \in \mathbf{Q}$ such that

$$
\begin{array}{lll}
\mathbf{a}(\overrightarrow{\boldsymbol{u}}, \overrightarrow{\boldsymbol{v}})+\mathbf{b}(\overrightarrow{\boldsymbol{v}}, \vec{p}) & =\mathbf{F}(\overrightarrow{\boldsymbol{v}}) & \forall \overrightarrow{\boldsymbol{v}}:=\left(\boldsymbol{v}_{\mathrm{B}}, \boldsymbol{z}_{\mathrm{B}}, \boldsymbol{v}_{\mathrm{D}}\right) \in \mathbf{H}, \\
\mathbf{b}(\overrightarrow{\boldsymbol{u}}, \vec{q}) & =\mathbf{G}(\vec{q}) & \forall \vec{q}:=\left(q_{\mathrm{B}}, q_{\mathrm{D}}, \xi\right) \in \mathbf{Q},
\end{array}
$$

where

$$
\mathbf{H}:=\mathbf{H}_{\mathrm{B}}\left(\operatorname{div} ; \Omega_{\mathrm{B}}\right) \times \mathbf{H}_{0}\left(\mathbf{c u r l} ; \Omega_{\mathrm{B}}\right) \times \mathbf{H}_{\mathrm{D}}\left(\operatorname{div} ; \Omega_{\mathrm{D}}\right), \quad \mathbf{Q}:=\mathrm{L}^{2}\left(\Omega_{\mathrm{B}}\right) \times \mathrm{L}^{2}\left(\Omega_{\mathrm{D}}\right) \times \mathrm{H}^{1 / 2}(\Sigma),
$$

$\mathbf{a}: \mathbf{H} \times \mathbf{H} \rightarrow \mathbb{R}$ and $\mathbf{b}: \mathbf{H} \times \mathbf{Q} \rightarrow \mathbb{R}$ are the bilinear forms defined by

$$
\begin{gathered}
\mathbf{a}(\overrightarrow{\boldsymbol{u}}, \overrightarrow{\boldsymbol{v}}):=\alpha \int_{\Omega_{\mathrm{B}}} \boldsymbol{u}_{\mathrm{B}} \cdot \boldsymbol{v}_{\mathrm{B}}+\nu \int_{\Omega_{\mathrm{B}}} \boldsymbol{\omega}_{\mathrm{B}} \cdot \boldsymbol{z}_{\mathrm{B}}+\nu \int_{\Omega_{\mathrm{B}}} \boldsymbol{v}_{\mathrm{B}} \cdot \operatorname{curl} \boldsymbol{\omega}_{\mathrm{B}} \\
-\nu \int_{\Omega_{\mathrm{B}}} \boldsymbol{u}_{\mathrm{B}} \cdot \operatorname{curl} \boldsymbol{z}_{\mathrm{B}}+\mu \int_{\Omega_{\mathrm{D}}} \boldsymbol{u}_{\mathrm{D}} \cdot \boldsymbol{v}_{\mathrm{D}} \quad \forall(\overrightarrow{\boldsymbol{u}}, \overrightarrow{\boldsymbol{v}}) \in \mathbf{H} \times \mathbf{H}, \\
\mathbf{b}(\overrightarrow{\boldsymbol{v}}, \vec{q}):=-\int_{\Omega_{\mathrm{B}}} q_{\mathrm{B}} \operatorname{div} \boldsymbol{v}_{\mathrm{B}}-\int_{\Omega_{\mathrm{D}}} q_{\mathrm{D}} \operatorname{div} \boldsymbol{v}_{\mathrm{D}}+\left\langle\boldsymbol{v}_{\mathrm{B}} \cdot \boldsymbol{n}-\boldsymbol{v}_{\mathrm{D}} \cdot \boldsymbol{n}, \xi\right\rangle_{\Sigma} \quad \forall(\overrightarrow{\boldsymbol{v}}, \vec{q}) \in \mathbf{H} \times \mathbf{Q},
\end{gathered}
$$

and $\mathbf{F} \in \mathbf{H}^{\prime}$ and $\mathbf{G} \in \mathbf{Q}^{\prime}$ are the functionals defined by

$$
\mathbf{F}(\overrightarrow{\boldsymbol{v}}):=\int_{\Omega_{\mathrm{B}}} \boldsymbol{f}_{\mathrm{B}} \cdot \boldsymbol{v}_{\mathrm{B}}+\int_{\Omega_{\mathrm{D}}} f_{\mathrm{D}} \cdot \boldsymbol{v}_{\mathrm{D}} \quad \forall \overrightarrow{\boldsymbol{v}} \in \mathbf{H}, \quad \text { and } \quad \mathbf{G}=\mathbf{0} .
$$




\section{Solvability analysis of the mixed formulation}

In this section we analyze the solvability of (2.11). For this purpose, we first recall some definitions and technical results concerning Sobolev spaces on $\Gamma_{\mathrm{D}}, \Gamma_{\mathrm{B}}$, and $\Sigma$. We begin by mentioning that, given $\eta \in \mathrm{H}^{-1 / 2}\left(\partial \Omega_{\mathrm{D}}\right)$, its restriction to $\Gamma_{\mathrm{D}}$, say $\left.\eta\right|_{\Gamma_{\mathrm{D}}}$, is defined as

$$
\left\langle\left.\eta\right|_{\Gamma_{\mathrm{D}}}, \rho\right\rangle_{\Gamma_{\mathrm{D}}}:=\left\langle\eta, E_{\mathrm{D}, 0}(\rho)\right\rangle_{\partial \Omega_{\mathrm{D}}} \quad \forall \rho \in \mathrm{H}_{00}^{1 / 2}\left(\Gamma_{\mathrm{D}}\right),
$$

where $E_{\mathrm{D}, 0}: \mathrm{H}^{1 / 2}\left(\Gamma_{\mathrm{D}}\right) \rightarrow \mathrm{L}^{2}\left(\partial \Omega_{\mathrm{D}}\right)$ is the extension by zero in $\Sigma:=\partial \Omega_{\mathrm{D}} \backslash \Gamma_{D}$, and

$$
\mathrm{H}_{00}^{1 / 2}\left(\Gamma_{\mathrm{D}}\right):=\left\{\rho \in \mathrm{H}^{1 / 2}\left(\Gamma_{\mathrm{D}}\right): \quad E_{\mathrm{D}, 0}(\rho) \in \mathrm{H}^{1 / 2}\left(\partial \Omega_{\mathrm{D}}\right)\right\},
$$

which is endowed with the natural norm $\|\rho\|_{1 / 2,00, \Gamma_{\mathrm{D}}}:=\left\|E_{\mathrm{D}, 0}(\rho)\right\|_{1 / 2, \partial \Omega_{\mathrm{D}}}$. It is quite clear, then, that $\left.\eta\right|_{\Gamma_{\mathrm{D}}}$ belongs to $\mathrm{H}_{00}^{-1 / 2}\left(\Gamma_{\mathrm{D}}\right)$, the dual of $\mathrm{H}_{00}^{1 / 2}\left(\Gamma_{\mathrm{D}}\right)$, and that $\eta=0$ on $\Gamma_{\mathrm{D}}$ (equivalently $\left.\eta\right|_{\Gamma_{\mathrm{D}}}=0$ ) if and only if

$$
\left\langle\eta, E_{\mathrm{D}, 0}(\rho)\right\rangle_{\partial \Omega_{\mathrm{D}}}=0 \quad \forall \rho \in \mathrm{H}_{00}^{1 / 2}\left(\Gamma_{\mathrm{D}}\right) .
$$

Hereafter, $\langle\cdot, \cdot\rangle_{\Gamma_{\mathrm{D}}}\left(\right.$ resp. $\langle\cdot, \cdot\rangle_{\partial \Omega_{\mathrm{D}}}$ ) stands for the duality pairing of the spaces $\mathrm{H}_{00}^{-1 / 2}\left(\Gamma_{\mathrm{D}}\right)$ and $\mathrm{H}_{00}^{1 / 2}\left(\Gamma_{\mathrm{D}}\right)$ (resp. $\mathrm{H}^{-1 / 2}\left(\partial \Omega_{\mathrm{D}}\right)$ and $\left.\mathrm{H}^{1 / 2}\left(\partial \Omega_{\mathrm{D}}\right)\right)$ with respect to the $\mathrm{L}^{2}\left(\Gamma_{\mathrm{D}}\right)$ (resp. $\left.\mathrm{L}^{2}\left(\partial \Omega_{\mathrm{D}}\right)\right)$ inner product. Furthermore, it is not difficult to show (see, e.g. [19, Section 2]) that there holds the decomposition

$$
H^{1 / 2}\left(\partial \Omega_{\mathrm{D}}\right)=E_{\mathrm{D}}\left(\mathrm{H}^{1 / 2}(\Sigma)\right) \oplus E_{\mathrm{D}, 0}\left(\mathrm{H}_{00}^{1 / 2}\left(\Gamma_{\mathrm{D}}\right)\right),
$$

where $E_{\mathrm{D}}: \mathrm{H}^{1 / 2}(\Sigma) \rightarrow \mathrm{H}^{1 / 2}\left(\partial \Omega_{\mathrm{D}}\right)$ is the bounded linear extension defined by $E_{\mathrm{D}}(\xi):=\left.z_{\xi}\right|_{\partial \Omega_{\mathrm{D}}} \quad \forall \xi \in$ $\mathrm{H}^{1 / 2}(\Sigma)$, with $z_{\xi} \in \mathrm{H}^{1}\left(\Omega_{\mathrm{D}}\right)$ being the unique weak solution of the boundary value problem with mixed boundary conditions:

$$
\Delta z_{\xi}=0 \quad \text { in } \quad \Omega_{\mathrm{D}}, \quad z_{\xi}=\xi \quad \text { on } \quad \Sigma, \quad \nabla z_{\xi} \cdot \boldsymbol{n}=0 \quad \text { on } \quad \Gamma_{\mathrm{D}} .
$$

In this way, given $\varphi \in \mathrm{H}^{1 / 2}\left(\partial \Omega_{\mathrm{D}}\right)$, there exist unique $\xi_{\varphi} \in \mathrm{H}^{1 / 2}(\Sigma)$ and $\rho_{\varphi} \in \mathrm{H}_{00}^{1 / 2}\left(\Gamma_{\mathrm{D}}\right)$ such that

$$
\varphi=E_{\mathrm{D}}\left(\xi_{\varphi}\right)+E_{\mathrm{D}, 0}\left(\rho_{\varphi}\right),
$$

and hence

$$
\langle\eta, \varphi\rangle_{\partial \Omega_{\mathrm{D}}}=\left\langle\eta, E_{\mathrm{D}}\left(\xi_{\varphi}\right)\right\rangle_{\partial \Omega_{\mathrm{D}}}+\left\langle\eta, E_{\mathrm{D}, 0}\left(\rho_{\varphi}\right)\right\rangle_{\partial \Omega_{\mathrm{D}}}
$$

which can be rewritten as

$$
\langle\eta, \varphi\rangle_{\partial \Omega_{\mathrm{D}}}=\left\langle\eta_{\Sigma}, \xi_{\varphi}\right\rangle_{\Sigma}+\left\langle\eta_{\mathrm{D}}, \rho_{\varphi}\right\rangle_{\Gamma_{\mathrm{D}}}
$$

where $\eta_{\Sigma} \in \mathrm{H}^{-1 / 2}(\Sigma)$ and $\eta_{\mathrm{D}} \in \mathrm{H}_{00}^{-1 / 2}\left(\Gamma_{\mathrm{D}}\right)$ are defined accordingly. In addition, it is clear from (3.1) and the definitions of $E_{\mathrm{D}}$ and $E_{\mathrm{D}, 0}$ that actually $\xi_{\varphi}=\left.\varphi\right|_{\Sigma}$ for each $\varphi \in \mathrm{H}^{1 / 2}\left(\partial \Omega_{\mathrm{D}}\right)$. In particular, when $\eta=0$ on $\Gamma_{\mathrm{D}}$, the foregoing equations yield $\langle\eta, \varphi\rangle_{\partial \Omega_{\mathrm{D}}}=\left\langle\eta, E_{\mathrm{D}}\left(\xi_{\varphi}\right)\right\rangle_{\partial \Omega_{\mathrm{D}}}=:\left\langle\eta_{\Sigma}, \xi_{\varphi}\right\rangle_{\Sigma}=\left\langle\eta_{\Sigma},\left.\varphi\right|_{\Sigma}\right\rangle_{\Sigma}$, and hence $\eta$ can be identified with a functional $\eta_{\Sigma} \in \mathrm{H}^{-1 / 2}(\Sigma)$. In other words, one simply says that $\left.\eta\right|_{\Sigma}=\eta_{\Sigma} \in \mathrm{H}^{-1 / 2}(\Sigma)$. Note that an interesting application of this result arises when we consider $\boldsymbol{v}_{\mathrm{D}} \in \mathbf{H}_{\mathrm{D}}\left(\operatorname{div} ; \Omega_{\mathrm{D}}\right)$ and define $\eta:=\boldsymbol{v}_{\mathrm{D}} \cdot \boldsymbol{n} \in \mathrm{H}^{-1 / 2}\left(\partial \Omega_{\mathrm{D}}\right)$. In fact, since $\boldsymbol{v}_{\mathrm{D}} \cdot \boldsymbol{n}=0$ on $\Gamma_{\mathrm{D}}$, we readily deduce that $\left.\boldsymbol{v}_{\mathrm{D}} \cdot \boldsymbol{n}\right|_{\Sigma} \in \mathrm{H}^{-1 / 2}(\Sigma)$. Moreover, the analogue conclusion obtained by exchanging $\Omega_{\mathrm{D}}, \Gamma_{\mathrm{D}}$, and $\mathbf{H}_{\mathrm{D}}\left(\operatorname{div} ; \Omega_{\mathrm{D}}\right)$ by $\Omega_{\mathrm{B}}, \Gamma_{\mathrm{B}}$, and $\mathbf{H}_{\mathrm{B}}\left(\right.$ div $\left.; \Omega_{\mathrm{B}}\right)$, respectively, is precisely what we used in Section 2 for the derivation of $(2.5)$.

We are now in position to provide the following preliminary result, which establishes a continuous inf-sup condition on $\mathbf{H}_{\mathrm{D}}\left(\operatorname{div} ; \Omega_{\mathrm{D}}\right) \times\left(\mathrm{L}_{0}^{2}\left(\Omega_{\mathrm{D}}\right) \times \mathrm{H}^{1 / 2}(\Sigma)\right)$.

Lemma 3.1. There exists $\beta_{\mathrm{D}}>0$ such that

$$
S_{\mathrm{D}}\left(q_{\mathrm{D}}, \xi\right):=\sup _{\substack{\boldsymbol{v}_{\mathrm{D}} \in \mathbf{H}_{\mathrm{D}}\left(\text { div; } ; \Omega_{\mathrm{D}}\right) \\ \boldsymbol{v}_{\mathrm{D}} \neq \mathbf{0}}} \frac{\int_{\Omega_{\mathrm{D}}} q_{\mathrm{D}} \operatorname{div} \boldsymbol{v}_{\mathrm{D}}+\left\langle\boldsymbol{v}_{\mathrm{D}} \cdot \boldsymbol{n}, \xi\right\rangle_{\Sigma}}{\left\|\boldsymbol{v}_{\mathrm{D}}\right\|_{\text {div } ; \Omega_{\mathrm{D}}}} \geq \beta_{\mathrm{D}}\left\{\left\|q_{\mathrm{D}}\right\|_{0, \Omega_{\mathrm{D}}}+\|\xi\|_{1 / 2, \Sigma}\right\}
$$

for all $\left(q_{\mathrm{D}}, \xi\right) \in \mathrm{L}_{0}^{2}\left(\Omega_{\mathrm{D}}\right) \times \mathrm{H}^{1 / 2}(\Sigma)$. 
Proof. It proceeds almost verbatim as the $2 \mathrm{D}$ version provided in [25, Lemma 3.3]. However, for sake of completeness, most details are given in what follows. The first part of the proof reduces to show that the operator div : $\mathbf{H}_{\mathrm{D}}\left(\operatorname{div} ; \Omega_{\mathrm{D}}\right) \rightarrow \mathrm{L}_{0}^{2}\left(\Omega_{\mathrm{D}}\right)$ is surjective, for which, given $q_{\mathrm{D}} \in \mathrm{L}_{0}^{2}\left(\Omega_{\mathrm{D}}\right)$, it suffices to define the pre-image $\widetilde{\boldsymbol{v}}_{\mathrm{D}}:=\nabla z \in \mathbf{H}_{\mathrm{D}}\left(\operatorname{div} ; \Omega_{\mathrm{D}}\right)$, where $z \in \mathrm{H}^{1}\left(\Omega_{\mathrm{D}}\right)$ is the unique weak solution of the Neumann boundary value problem

$$
\Delta z=q_{\mathrm{D}} \quad \text { in } \quad \Omega_{\mathrm{D}}, \quad \nabla z \cdot \boldsymbol{n}=0 \quad \text { on } \quad \partial \Omega_{\mathrm{D}}, \quad \int_{\Omega_{\mathrm{D}}} z=0 .
$$

Indeed, it is clear that $\operatorname{div} \widetilde{\boldsymbol{v}}_{\mathrm{D}}=\Delta z=q_{\mathrm{D}}$ in $\Omega_{\mathrm{D}}$, and the continuous dependence result for (3.4) establishes that $\left\|\widetilde{\boldsymbol{v}}_{\mathrm{D}}\right\|_{0, \Omega_{\mathrm{D}}}=|z|_{1, \Omega_{\mathrm{D}}} \leq\|z\|_{1, \Omega_{\mathrm{D}}} \leq c\left\|q_{\mathrm{D}}\right\|_{0, \Omega_{\mathrm{D}}}$, whence we readily deduce that $\left\|\widetilde{\boldsymbol{v}}_{\mathrm{D}}\right\|_{\text {div } ; \Omega_{\mathrm{D}}} \leq \widetilde{C}\left\|q_{\mathrm{D}}\right\|_{0, \Omega_{\mathrm{D}}}$. In this way, since

$$
S_{\mathrm{D}}\left(q_{\mathrm{D}}, \xi\right) \geq \sup _{\substack{\boldsymbol{v}_{\mathrm{D}} \in \mathbf{H}_{\mathrm{D}}\left(\text { div } ; \Omega_{\mathrm{D}}\right) \\ \boldsymbol{v}_{\mathrm{D}} \neq \mathbf{0}}} \frac{\int_{\Omega_{\mathrm{D}}} q_{\mathrm{D}} \operatorname{div} \boldsymbol{v}_{\mathrm{D}}}{\left\|\boldsymbol{v}_{\mathrm{D}}\right\|_{\operatorname{div} ; \Omega_{\mathrm{D}}}}-\|\xi\|_{1 / 2, \Sigma} \geq \frac{\left|\int_{\Omega_{\mathrm{D}}} q_{\mathrm{D}} \operatorname{div} \widetilde{\boldsymbol{v}}_{\mathrm{D}}\right|}{\left\|\widetilde{\boldsymbol{v}}_{\mathrm{D}}\right\|_{\text {div } ; \Omega_{\mathrm{D}}}}-\|\xi\|_{1 / 2, \Sigma},
$$

the foregoing identity and estimates imply the existence of $C>0$ such that

$$
S_{\mathrm{D}}\left(q_{\mathrm{D}}, \xi\right) \geq C\left\|q_{\mathrm{D}}\right\|_{0, \Omega_{\mathrm{D}}}-\|\xi\|_{1 / 2, \Sigma}
$$

In turn, the main ingredient of the second part has to do with the construction of a proper extension of an arbitrary $\phi \in \mathrm{H}^{-1 / 2}(\Sigma)$ to a functional $\eta \in \mathrm{H}^{-1 / 2}\left(\partial \Omega_{\mathrm{D}}\right)$. More precisely, given $\xi \in \mathrm{H}^{1 / 2}(\Sigma)$, we consider $\phi \in \mathrm{H}^{-1 / 2}(\Sigma)$ and, following the previous analysis and notations, we simply define $\eta \in \mathrm{H}^{-1 / 2}\left(\partial \Omega_{\mathrm{D}}\right)$ as

$$
\langle\eta, \varphi\rangle_{\partial \Omega_{\mathrm{D}}}:=\left\langle\phi, \xi_{\varphi}\right\rangle_{\Sigma}=\left\langle\phi,\left.\varphi\right|_{\Sigma}\right\rangle_{\Sigma} \quad \forall \varphi \in \mathrm{H}^{1 / 2}\left(\partial \Omega_{\mathrm{D}}\right),
$$

which yields $\|\eta\|_{-1 / 2, \partial \Omega_{\mathrm{D}}} \leq\|\phi\|_{-1 / 2, \Sigma}$. It follows straightforwardly from (3.1) and (3.2) that

$$
\left\langle\eta, E_{\mathrm{D}, 0}(\rho)\right\rangle_{\partial \Omega_{\mathrm{D}}}=0 \quad \forall \rho \in \mathrm{H}_{00}^{1 / 2}\left(\Gamma_{\mathrm{D}}\right) \quad \text { and } \quad\left\langle\eta, E_{\mathrm{D}}(\xi)\right\rangle_{\partial \Omega_{\mathrm{D}}}=\langle\phi, \xi\rangle_{\Sigma} \quad \forall \xi \in \mathrm{H}^{1 / 2}(\Sigma),
$$

which says, equivalently, that $\eta=0$ on $\Gamma_{\mathrm{D}}$ and $\eta=\phi$ on $\Sigma$. Next, we let $z_{\eta} \in \mathrm{H}^{1}\left(\Omega_{\mathrm{D}}\right)$ be the unique weak solution of the boundary value problem

$$
\Delta z_{\eta}=\frac{1}{\left|\Omega_{\mathrm{D}}\right|}\langle\eta, 1\rangle_{\partial \Omega_{\mathrm{D}}} \quad \text { in } \quad \Omega_{\mathrm{D}}, \quad \nabla z_{\eta} \cdot \boldsymbol{n}=\eta \quad \text { on } \quad \partial \Omega_{\mathrm{D}}, \quad \int_{\Omega_{\mathrm{D}}} z_{\eta}=0,
$$

define $\boldsymbol{w}_{\mathrm{D}}:=\nabla z_{\eta}$ in $\Omega_{\mathrm{D}}$, and observe that $\operatorname{div} \boldsymbol{w}_{\mathrm{D}}=\frac{1}{\left|\Omega_{\mathrm{D}}\right|}\langle\eta, 1\rangle_{\partial \Omega_{\mathrm{D}}} \in P_{0}\left(\Omega_{\mathrm{D}}\right)$ (which yields $\boldsymbol{w}_{\mathrm{D}} \in$ $\left.\mathbf{H}\left(\operatorname{div} ; \Omega_{\mathrm{D}}\right)\right), \quad \boldsymbol{w}_{\mathrm{D}} \cdot n=\eta$ on $\partial \Omega_{\mathrm{D}}$, and $\left\|\boldsymbol{w}_{\mathrm{D}}\right\|_{\text {div } ; \Omega_{\mathrm{D}}} \leq C\|\eta\|_{-1 / 2, \partial \Omega_{\mathrm{D}}} \leq C\|\phi\|_{-1 / 2, \Sigma}$. It follows that $\boldsymbol{w}_{\mathrm{D}} \in \mathbf{H}_{\mathrm{D}}\left(\operatorname{div} ; \Omega_{\mathrm{D}}\right)$ and hence

$$
S_{\mathrm{D}}\left(q_{\mathrm{D}}, \xi\right) \geq \frac{\left|\int_{\Omega_{\mathrm{D}}} q_{\mathrm{D}} \operatorname{div} \boldsymbol{w}_{\mathrm{D}}+\left\langle\boldsymbol{w}_{\mathrm{D}} \cdot \boldsymbol{n}, \xi\right\rangle_{\Sigma}\right|}{\left\|\boldsymbol{w}_{\mathrm{D}}\right\|_{\text {div } ; \Omega_{\mathrm{D}}}}=\frac{\left|\langle\phi, \xi\rangle_{\Sigma}\right|}{\left\|\boldsymbol{w}_{\mathrm{D}}\right\|_{\operatorname{div} ; \Omega_{\mathrm{D}}}} \geq c \frac{\left|\langle\phi, \xi\rangle_{\Sigma}\right|}{\|\phi\|_{-1 / 2, \Sigma}},
$$

which, being valid for any $\phi \in \mathrm{H}^{-1 / 2}(\Sigma)$, implies that $S_{\mathrm{D}}\left(q_{\mathrm{D}}, \xi\right) \geq c\|\xi\|_{1 / 2, \Sigma}$. This inequality and (3.5) yield (3.3), thus completing the proof.

The following result is basically a "mirror reflection" through $\Sigma$ of the previous lemma.

Lemma 3.2. There exists $\beta_{\mathrm{B}}>0$ such that

$$
S_{\mathrm{B}}\left(q_{\mathrm{B}}, \xi\right):=\sup _{\substack{\boldsymbol{v}_{\mathrm{B}} \in \mathbf{H}_{\mathrm{B}}\left(\text { div } ; \Omega_{\mathrm{B}}\right) \\ \boldsymbol{v}_{\mathrm{B}} \neq \mathbf{0}}} \frac{\int_{\Omega_{\mathrm{B}}} q_{\mathrm{B}} \operatorname{div} \boldsymbol{v}_{\mathrm{B}}-\left\langle\boldsymbol{v}_{\mathrm{B}} \cdot \boldsymbol{n}, \xi\right\rangle_{\Sigma}}{\left\|\boldsymbol{v}_{\mathrm{B}}\right\|_{\text {div } ; \Omega_{\mathrm{B}}}} \geq \beta_{\mathrm{B}}\left\{\left\|q_{\mathrm{B}}\right\|_{0, \Omega_{\mathrm{B}}}+\|\xi\|_{1 / 2, \Sigma}\right\}
$$

for all $\left(q_{\mathrm{B}}, \xi\right) \in \mathrm{L}_{0}^{2}\left(\Omega_{\mathrm{B}}\right) \times \mathrm{H}^{1 / 2}(\Sigma)$. 
Proof. It proceeds exactly as the proof of Lemma 3.1 by replacing $\Omega_{\mathrm{D}}, \Gamma_{\mathrm{D}}$, and $\mathbf{H}_{\mathrm{D}}\left(\right.$ div $\left.; \Omega_{\mathrm{D}}\right)$ by $\Omega_{\mathrm{B}}, \Gamma_{\mathrm{B}}$, and $\mathbf{H}_{\mathrm{B}}\left(\operatorname{div} ; \Omega_{\mathrm{B}}\right)$, respectively.

Lemma 3.1 and 3.2 imply the following continuous inf-sup condition for $\mathbf{b}$.

Lemma 3.3. There exists $\beta>0$ such that

$$
S(\vec{q}):=\sup _{\substack{\overrightarrow{\boldsymbol{v}} \in \mathbf{H} \\ \overrightarrow{\boldsymbol{v}} \neq \mathbf{0}}} \frac{\mathbf{b}(\overrightarrow{\boldsymbol{v}}, \vec{q})}{\|\overrightarrow{\boldsymbol{v}}\|_{\mathbf{H}}} \geq \beta\left\{\left\|q_{\mathrm{B}, 0}\right\|_{0, \Omega_{\mathrm{B}}}+\left\|q_{\mathrm{D}, 0}\right\|_{0, \Omega_{\mathrm{D}}}+\left\|\xi-c_{\mathrm{B}}\right\|_{1 / 2, \Sigma}+\left\|\xi-c_{\mathrm{D}}\right\|_{1 / 2, \Sigma}\right\}
$$

for all $\vec{q}:=\left(q_{\mathrm{B}}, q_{\mathrm{D}}, \xi\right) \in \mathbf{Q}$, where, according to $(1.1), q_{\mathrm{B}}=q_{\mathrm{B}, 0}+c_{\mathrm{B}}$ and $q_{\mathrm{D}}=q_{\mathrm{D}, 0}+c_{\mathrm{D}}$, with $q_{\mathrm{B}, 0} \in \mathrm{L}_{0}^{2}\left(\Omega_{\mathrm{B}}\right)$, $q_{\mathrm{D}, 0} \in \mathrm{L}_{0}^{2}\left(\Omega_{\mathrm{D}}\right)$, and $c_{\mathrm{B}}:=\frac{1}{\left|\Omega_{\mathrm{B}}\right|} \int_{\Omega_{\mathrm{B}}} q_{\mathrm{B}}, c_{\mathrm{D}}:=\frac{1}{\left|\Omega_{\mathrm{D}}\right|} \int_{\Omega_{\mathrm{D}}} q_{\mathrm{D}} \in \mathbb{R}$.

Proof. Given $\vec{q}:=\left(q_{\mathrm{B}}, q_{\mathrm{D}}, \xi\right) \in \mathbf{Q}$, with $q_{\mathrm{B}}$ and $q_{\mathrm{D}}$ decomposed as indicated above, we integrate by parts in $\Omega_{\mathrm{B}}$ and $\Omega_{\mathrm{D}}$, respectively, to deduce that

$$
\int_{\Omega_{\mathrm{B}}} q_{\mathrm{B}} \operatorname{div} \boldsymbol{v}_{\mathrm{B}}-\left\langle\boldsymbol{v}_{\mathrm{B}} \cdot \boldsymbol{n}, \xi\right\rangle_{\Sigma}=\int_{\Omega_{\mathrm{B}}} q_{\mathrm{B}, 0} \operatorname{div} \boldsymbol{v}_{\mathrm{B}}-\left\langle\boldsymbol{v}_{\mathrm{B}} \cdot \boldsymbol{n}, \xi-c_{\mathrm{B}}\right\rangle_{\Sigma} \quad \forall \boldsymbol{v}_{\mathrm{B}} \in \mathbf{H}_{\mathrm{B}}\left(\operatorname{div} ; \Omega_{\mathrm{B}}\right),
$$

and

$$
\int_{\Omega_{\mathrm{D}}} q_{\mathrm{D}} \operatorname{div} \boldsymbol{v}_{\mathrm{D}}+\left\langle\boldsymbol{v}_{\mathrm{D}} \cdot \boldsymbol{n}, \xi\right\rangle_{\Sigma}=\int_{\Omega_{\mathrm{D}}} q_{\mathrm{D}, 0} \operatorname{div} \boldsymbol{v}_{\mathrm{D}}+\left\langle\boldsymbol{v}_{\mathrm{D}} \cdot \boldsymbol{n}, \xi-c_{\mathrm{D}}\right\rangle_{\Sigma} \quad \forall \boldsymbol{v}_{\mathrm{D}} \in \mathbf{H}_{\mathrm{D}}\left(\operatorname{div} ; \Omega_{\mathrm{D}}\right) .
$$

Hence, bearing in mind the definitions of the bilinear form $\mathbf{b}$ (cf. (2.13)) and the operators $S_{\mathrm{D}}$ and $S_{\mathrm{B}}$ (cf. (3.3), (3.6)), and employing the foregoing equations, we easily find that

$$
S(\vec{q}) \geq S_{\mathrm{D}}\left(q_{\mathrm{D}, 0}, \xi-c_{\mathrm{D}}\right) \quad \text { and } \quad S(\vec{q}) \geq S_{\mathrm{B}}\left(q_{\mathrm{B}, 0}, \xi-c_{\mathrm{B}}\right)
$$

Consequently, these inequalities, along with straightforward applications of Lemmata 3.1 and 3.2, imply (3.7) and complete the proof.

Having proved a first property for $\mathbf{b}$, we now observe that the bilinear form a satisfies a positiveness condition. More precisely, it follows directly from its definition (cf. (2.12)) that

$$
\mathbf{a}(\overrightarrow{\boldsymbol{v}}, \overrightarrow{\boldsymbol{v}})=\alpha\left\|\boldsymbol{v}_{\mathrm{B}}\right\|_{0, \Omega_{\mathrm{B}}}^{2}+\nu\left\|\boldsymbol{z}_{\mathrm{B}}\right\|_{0, \Omega_{\mathrm{B}}}^{2}+\mu\left\|\boldsymbol{v}_{\mathrm{D}}\right\|_{0, \Omega_{\mathrm{D}}}^{2} \quad \forall \overrightarrow{\boldsymbol{v}}:=\left(\boldsymbol{v}_{\mathrm{B}}, \boldsymbol{z}_{\mathrm{B}}, \boldsymbol{v}_{\mathrm{D}}\right) \in \mathbf{H}
$$

A first result concerning the solvability of our mixed formulation (2.11) is established next.

Theorem 3.1. Let $(\overrightarrow{\boldsymbol{u}}, \vec{p}):=\left(\left(\boldsymbol{u}_{\mathrm{B}}, \boldsymbol{\omega}_{\mathrm{B}}, \boldsymbol{u}_{\mathrm{D}}\right),\left(p_{\mathrm{B}}, p_{\mathrm{D}}, \lambda\right)\right) \in \mathbf{H} \times \mathbf{Q}$ be a solution of the homogeneous problem associated to (2.11), that is with $\mathbf{F}=\mathbf{G}=\mathbf{0}$. Then $\overrightarrow{\boldsymbol{u}}=\mathbf{0}$ and there exists $c \in \mathbb{R}$ such that $\vec{p}=(c, c, c)$.

Proof. We first notice from the second equation of (2.11) with $\vec{q}=\vec{p}$ that $\mathbf{b}(\overrightarrow{\boldsymbol{u}}, \vec{p})=0$, and hence, taking $\overrightarrow{\boldsymbol{v}}=\overrightarrow{\boldsymbol{u}}$ in the first equation of (2.11) and using the identity (3.8), we arrive at

$$
0=\mathbf{a}(\overrightarrow{\boldsymbol{u}}, \overrightarrow{\boldsymbol{u}})=\alpha\left\|\boldsymbol{u}_{\mathrm{B}}\right\|_{0, \Omega_{\mathrm{B}}}^{2}+\nu\left\|\boldsymbol{\omega}_{\mathrm{B}}\right\|_{0, \Omega_{\mathrm{B}}}^{2}+\mu\left\|\boldsymbol{u}_{\mathrm{D}}\right\|_{0, \Omega_{\mathrm{D}}}^{2}
$$

from which it follows that $\overrightarrow{\boldsymbol{u}}=\mathbf{0}$. In this way, the first equation of (2.11) becomes now $\mathbf{b}(\overrightarrow{\boldsymbol{v}}, \vec{p})=0$ for all $\overrightarrow{\boldsymbol{v}} \in \mathbf{Q}$, which, according to the continuous inf-sup condition for $\mathbf{b}$ given by Lemma 3.3, yields $p_{\mathrm{B}, 0}=0$, $p_{\mathrm{D}, 0}=0$, and $\lambda=\frac{1}{\left|\Omega_{\mathrm{B}}\right|} \int_{\Omega_{\mathrm{B}}} p_{\mathrm{B}}=\frac{1}{\left|\Omega_{\mathrm{D}}\right|} \int_{\Omega_{\mathrm{D}}} p_{\mathrm{D}}=: c \in \mathbb{R}$, so that $p_{\mathrm{B}}=p_{\mathrm{B}, 0}+c=c$ and $p_{\mathrm{D}}=p_{\mathrm{D}, 0}+c=c$.

As a straightforward consequence of Theorem 3.1 we conclude that whenever (2.11) has solution, it is not unique. Therefore, in order to overcome this drawback, we need to remove the constant $c \in \mathbb{R}$ from the 
solutions of the associated homogeneous system, for which from now on we propose to look for the unknown $\vec{p}$ in the space

$$
\mathbf{Q}_{0}:=\mathrm{L}_{0}^{2}\left(\Omega_{\mathrm{B}}\right) \times \mathrm{L}^{2}\left(\Omega_{\mathrm{D}}\right) \times \mathrm{H}^{1 / 2}(\Sigma) .
$$

Alternatively, one could also consider $\mathbf{Q}_{0}:=\mathrm{L}^{2}\left(\Omega_{\mathrm{B}}\right) \times \mathrm{L}_{0}^{2}\left(\Omega_{\mathrm{D}}\right) \times \mathrm{H}^{1 / 2}(\Sigma)$ or $\mathbf{Q}_{0}:=\mathrm{L}^{2}\left(\Omega_{\mathrm{B}}\right) \times \mathrm{L}^{2}\left(\Omega_{\mathrm{D}}\right) \times$ $\mathrm{H}_{0}^{1 / 2}(\Sigma)$, where $\mathrm{H}_{0}^{1 / 2}(\Sigma):=\left\{\xi \in \mathrm{H}^{1 / 2}(\Sigma): \quad\langle 1, \xi\rangle_{\Sigma}=0\right\}$.

Throughout the rest of the paper we stay with (3.9) and consider, instead of (2.11), the following mixed formulation: Find $\overrightarrow{\boldsymbol{u}}:=\left(\boldsymbol{u}_{\mathrm{B}}, \boldsymbol{\omega}_{\mathrm{B}}, \boldsymbol{u}_{\mathrm{D}}\right) \in \mathbf{H}$ and $\vec{p}:=\left(p_{\mathrm{B}}, p_{\mathrm{D}}, \lambda\right) \in \mathbf{Q}_{0}$ such that

$$
\begin{array}{lll}
\mathbf{a}(\overrightarrow{\boldsymbol{u}}, \overrightarrow{\boldsymbol{v}})+\mathbf{b}(\overrightarrow{\boldsymbol{v}}, \vec{p}) & =\mathbf{F}(\overrightarrow{\boldsymbol{v}}) & \forall \overrightarrow{\boldsymbol{v}}:=\left(\boldsymbol{v}_{\mathrm{B}}, \boldsymbol{z}_{\mathrm{B}}, \boldsymbol{v}_{\mathrm{D}}\right) \in \mathbf{H}, \\
\mathbf{b}(\overrightarrow{\boldsymbol{u}}, \vec{q}) & =\mathbf{G}(\vec{q}) & \forall \vec{q}:=\left(q_{\mathrm{B}}, q_{\mathrm{D}}, \xi\right) \in \mathbf{Q}_{0} .
\end{array}
$$

Note that the second equation of (2.11), which is tested against $\vec{q} \in \mathbf{Q}$, is equivalent to the present second equation of (3.10), which is tested against $\vec{q} \in \mathbf{Q}_{0}$. In fact, one implication is obvious because of the inclusion $\mathbf{Q}_{0} \subseteq \mathbf{Q}$. Conversely, assume that the second equation of (3.10) holds. Then, given $c \in \mathbb{R}$, we integrate by parts and, noting that $\left(\mathbf{0}, q_{\mathrm{D}}-c, \xi-c\right) \in \mathbf{Q}_{0}:=\mathrm{L}_{0}^{2}\left(\Omega_{\mathrm{B}}\right) \times \mathrm{L}^{2}\left(\Omega_{\mathrm{D}}\right) \times \mathrm{H}^{1 / 2}(\Sigma)$, we find that

$$
\mathbf{b}\left(\overrightarrow{\boldsymbol{u}},\left(c, q_{\mathrm{D}}, \xi\right)\right)=\mathbf{b}\left(\overrightarrow{\boldsymbol{u}},\left(\mathbf{0}, q_{\mathrm{D}}-c, \xi-c\right)\right)=0,
$$

which yields $\mathbf{b}\left(\overrightarrow{\boldsymbol{u}},\left(q_{\mathrm{B}}, q_{\mathrm{D}}, \xi\right)\right)=0=\mathbf{G}(\vec{q})$ for all $\vec{q}:=\left(q_{\mathrm{B}}, q_{\mathrm{D}}, \xi\right) \in \mathbf{Q}$, thus confirming that the second equation of (2.11) holds.

We now aim to establish the well-posedness of (3.10) by applying the classical Babuška-Brezzi theory. We begin with the inf-sup condition for $\mathbf{b}$ on $\mathbf{H} \times \mathbf{Q}_{0}$.

Lemma 3.4. There exists $\widetilde{\beta}>0$ such that

$$
S(\vec{q}):=\sup _{\substack{\overrightarrow{\boldsymbol{v}} \in \mathbf{H} \\ \overrightarrow{\boldsymbol{v}} \neq \mathbf{0}}} \frac{\mathbf{b}(\overrightarrow{\boldsymbol{v}}, \vec{q})}{\|\overrightarrow{\boldsymbol{v}}\|_{\mathbf{H}}} \geq \widetilde{\beta}\|\vec{q}\|_{\mathbf{Q}} \quad \forall \vec{q} \in \mathbf{Q}_{0} .
$$

Proof. Given $\vec{q}=\left(q_{\mathrm{B}}, q_{\mathrm{D}}, \xi\right) \in \mathbf{Q}_{0}:=\mathrm{L}_{0}^{2}\left(\Omega_{\mathrm{B}}\right) \times \mathrm{L}^{2}\left(\Omega_{\mathrm{D}}\right) \times \mathrm{H}^{1 / 2}(\Sigma)$, we obtain from Lemma 3.3 that

$$
S(\vec{q}):=\sup _{\substack{\overrightarrow{\boldsymbol{v}} \in \mathbf{H} \\ \vec{v} \neq \mathbf{0}}} \frac{\mathbf{b}(\overrightarrow{\boldsymbol{v}}, \vec{q})}{\|\overrightarrow{\boldsymbol{v}}\|_{\mathbf{H}}} \geq \beta\left\{\left\|q_{\mathrm{B}}\right\|_{0, \Omega_{\mathrm{B}}}+\left\|q_{\mathrm{D}, 0}\right\|_{0, \Omega_{\mathrm{D}}}+\|\xi\|_{1 / 2, \Sigma}+\left\|\xi-c_{\mathrm{D}}\right\|_{1 / 2, \Sigma}\right\},
$$

where, according to (1.1), $q_{\mathrm{D}}=q_{\mathrm{D}, 0}+c_{\mathrm{D}}$, with $q_{\mathrm{D}, 0} \in \mathrm{L}_{0}^{2}\left(\Omega_{\mathrm{D}}\right)$ and $c_{\mathrm{D}}:=\frac{1}{\left|\Omega_{\mathrm{D}}\right|} \int_{\Omega_{\mathrm{D}}} q_{\mathrm{D}} \in \mathbb{R}$. In turn, a simple application of the triangle inequality shows that

$$
|\Sigma|\left|c_{\mathrm{D}}\right|=\left\|c_{\mathrm{D}}\right\|_{1 / 2, \Sigma} \leq\|\xi\|_{1 / 2, \Sigma}+\left\|\xi-c_{\mathrm{D}}\right\|_{1 / 2, \Sigma},
$$

which, combined with (3.12) and the fact that $\left\|q_{\mathrm{D}}\right\|_{0, \Omega_{\mathrm{D}}}^{2}=\left\|q_{\mathrm{D}, 0}\right\|_{0, \Omega_{\mathrm{D}}}^{2}+\left|\Omega_{\mathrm{D}}\right| c_{\mathrm{D}}^{2}$ (cf. (1.4)), imply (3.11) and finish the proof.

Next, we address the coerciveness of $\mathbf{a}$ on the kernel $\mathbf{V}$ of $\mathbf{b}$. Indeed, we first deduce from the definitions of $\mathbf{b}$ (cf. (2.13)) and $\mathbf{Q}_{0}$ (cf. (3.9)) that

$$
\mathbf{V}=\mathbf{V}_{\mathrm{B}, \mathrm{D}} \cap \mathbf{V}_{\Sigma},
$$

with

$$
\mathbf{V}_{\mathrm{B}, \mathrm{D}}:=\left\{\overrightarrow{\boldsymbol{v}}:=\left(\boldsymbol{v}_{\mathrm{B}}, \boldsymbol{z}_{\mathrm{B}}, \boldsymbol{v}_{\mathrm{D}}\right) \in \mathbf{H}: \quad \operatorname{div} \boldsymbol{v}_{\mathrm{B}} \in P_{0}\left(\Omega_{\mathrm{B}}\right) \quad \text { and } \quad \operatorname{div} \boldsymbol{v}_{\mathrm{D}}=0 \text { in } \Omega_{\mathrm{D}}\right\},
$$

and

$$
\mathbf{V}_{\Sigma}:=\left\{\overrightarrow{\boldsymbol{v}}:=\left(\boldsymbol{v}_{\mathrm{B}}, \boldsymbol{z}_{\mathrm{B}}, \boldsymbol{v}_{\mathrm{D}}\right) \in \mathbf{H}: \quad \boldsymbol{v}_{\mathrm{B}} \cdot \boldsymbol{n}=\boldsymbol{v}_{\mathrm{D}} \cdot \boldsymbol{n} \quad \text { on } \quad \Sigma\right\}
$$


Lemma 3.5. There exists $\widetilde{\varrho}>0$ such that

$$
\sup _{\substack{\boldsymbol{w} \in \mathbf{V} \\ \boldsymbol{w} \neq \mathbf{0}}} \frac{\mathbf{a}(\overrightarrow{\boldsymbol{v}}, \overrightarrow{\boldsymbol{w}})}{\|\overrightarrow{\boldsymbol{w}}\|_{\mathbf{H}}} \geq \widetilde{\varrho}\left\|_{\boldsymbol{v}}\right\|_{\mathbf{H}} \quad \forall \overrightarrow{\boldsymbol{v}} \in \mathbf{V}
$$

and

$$
\sup _{\substack{\overrightarrow{\boldsymbol{v}} \in \mathbf{V} \\ \boldsymbol{v} \neq \mathbf{0}}} \frac{\mathbf{a}(\overrightarrow{\boldsymbol{v}}, \overrightarrow{\boldsymbol{w}})}{\|\overrightarrow{\boldsymbol{v}}\|_{\mathbf{H}}} \geq \widetilde{\varrho}\|\overrightarrow{\boldsymbol{w}}\|_{\mathbf{H}} \quad \forall \overrightarrow{\boldsymbol{w}} \in \mathbf{V}
$$

Proof. We begin by recalling from [24, Lemma 3.2] that there exists $\varrho_{0}>0$ such that

$$
\left\|\boldsymbol{v}_{\mathrm{B}}\right\|_{0, \Omega_{\mathrm{B}}} \geq \varrho_{0}\left\|\boldsymbol{v}_{\mathrm{B}}\right\|_{\operatorname{div}, \Omega_{\mathrm{B}}} \forall \boldsymbol{v}_{\mathrm{B}} \in \mathbf{H}\left(\operatorname{div} ; \Omega_{\mathrm{B}}\right) \text { such that } \operatorname{div} \boldsymbol{v}_{\mathrm{B}} \in P_{0}\left(\Omega_{\mathrm{B}}\right) .
$$

Hence, thanks to the foregoing inequality and (3.8), we find that

$$
\mathbf{a}(\overrightarrow{\boldsymbol{v}}, \overrightarrow{\boldsymbol{v}}) \geq \widetilde{\varrho}_{1}\left\{\left\|\boldsymbol{v}_{\mathrm{B}}\right\|_{\mathrm{div} ; \Omega_{\mathrm{B}}}^{2}+\left\|\boldsymbol{z}_{\mathrm{B}}\right\|_{0, \Omega_{\mathrm{B}}}^{2}+\left\|\boldsymbol{v}_{\mathrm{D}}\right\|_{\mathrm{div} ; \Omega_{\mathrm{D}}}^{2}\right\} \quad \forall \overrightarrow{\boldsymbol{v}}:=\left(\boldsymbol{v}_{\mathrm{B}}, \boldsymbol{z}_{\mathrm{B}}, \boldsymbol{v}_{\mathrm{D}}\right) \in \mathbf{V}_{\mathrm{B}, \mathrm{D}}
$$

with $\widetilde{\varrho}_{1}:=\min \left\{\alpha \varrho_{0}^{2}, \nu, \mu\right\}>0$. Next, given a particular $\overrightarrow{\boldsymbol{v}}:=\left(\boldsymbol{v}_{\mathrm{B}}, \boldsymbol{z}_{\mathrm{B}}, \boldsymbol{v}_{\mathrm{D}}\right) \in \mathbf{V}$, we certainly have $\boldsymbol{z}_{\mathrm{B}} \in$ $\mathbf{H}_{0}\left(\mathbf{c u r l} ; \Omega_{\mathrm{B}}\right)$, and thus, due to a well-known result (see, e.g. [27, Chapter I, Section 2.3, Remark 2.5]), there holds curl $\boldsymbol{z}_{\mathrm{B}} \in \mathbf{H}_{0}\left(\operatorname{div} ; \Omega_{\mathrm{B}}\right)$, where

$$
\mathbf{H}_{0}\left(\operatorname{div} ; \Omega_{\mathrm{B}}\right):=\left\{\boldsymbol{v}_{\mathrm{B}} \in \mathbf{H}\left(\operatorname{div} ; \Omega_{\mathrm{B}}\right): \quad \boldsymbol{v}_{\mathrm{B}} \cdot \boldsymbol{n}=0 \quad \text { on } \quad \partial \Omega_{\mathrm{B}}\right\} .
$$

In this way, denoting

$$
T_{0}(\overrightarrow{\boldsymbol{v}}):=\left(\operatorname{curl} \boldsymbol{z}_{\mathrm{B}}, \boldsymbol{z}_{\mathrm{B}}, \mathbf{0}\right),
$$

which clearly belongs to $\mathbf{V}$, we find, according to the definition of a (cf. (2.12)), that

$$
\mathbf{a}\left(\overrightarrow{\boldsymbol{v}}, T_{0}(\overrightarrow{\boldsymbol{v}})\right)=(\alpha-\nu) \int_{\Omega_{\mathrm{B}}} \boldsymbol{v}_{\mathrm{B}} \cdot \operatorname{curl} \boldsymbol{z}_{\mathrm{B}}+\nu\left\|\operatorname{curl} \boldsymbol{z}_{\mathrm{B}}\right\|_{0, \Omega_{\mathrm{B}}}^{2}+\nu\left\|\boldsymbol{z}_{\mathrm{B}}\right\|_{0, \Omega_{\mathrm{B}}}^{2},
$$

which, applying Cauchy-Schwarz's inequality and simple algebraic manipulations, yields

$$
\mathbf{a}\left(\overrightarrow{\boldsymbol{v}}, T_{0}(\overrightarrow{\boldsymbol{v}})\right) \geq-\frac{|\alpha-\nu|^{2}}{2 \nu}\left\|\boldsymbol{v}_{\mathrm{B}}\right\|_{0, \Omega_{\mathrm{B}}}^{2}+\frac{\nu}{2}\left\|\mathbf{c u r l} \boldsymbol{z}_{\mathrm{B}}\right\|_{0, \Omega_{\mathrm{B}}}^{2}+\nu\left\|\boldsymbol{z}_{\mathrm{B}}\right\|_{0, \Omega_{\mathrm{B}}}^{2} .
$$

Therefore, introducing now $T(\overrightarrow{\boldsymbol{v}}):=c \overrightarrow{\boldsymbol{v}}+c_{0} T_{0}(\overrightarrow{\boldsymbol{v}})$, with suitable chosen positive constants $c$ and $c_{0}$ (depending on $\widetilde{\varrho}_{1}, \alpha$, and $\nu$ ), and utilizing (3.19) and (3.20), we obtain that

$$
T(\overrightarrow{\boldsymbol{v}}) \in \mathbf{V}, \quad\|T(\overrightarrow{\boldsymbol{v}})\|_{\mathbf{H}} \leq C\|\overrightarrow{\boldsymbol{v}}\|_{\mathbf{H}}, \quad \text { and } \quad \mathbf{a}(\overrightarrow{\boldsymbol{v}}, T(\overrightarrow{\boldsymbol{v}})) \geq \widetilde{\varrho}_{2}\|\overrightarrow{\boldsymbol{v}}\|_{\mathbf{H}}^{2},
$$

with $C$ and $\widetilde{\varrho}_{2}$ positive constants depending on $\widetilde{\varrho}_{1}, \alpha$, and $\nu$ as well. Then, we can write

$$
\sup _{\substack{\vec{w} \in \mathbf{V} \\ \boldsymbol{w} \neq \mathbf{0}}} \frac{\mathbf{a}(\overrightarrow{\boldsymbol{v}}, \overrightarrow{\boldsymbol{w}})}{\|\overrightarrow{\boldsymbol{w}}\|_{\mathbf{H}}} \geq \frac{\mathbf{a}(\overrightarrow{\boldsymbol{v}}, T(\overrightarrow{\boldsymbol{v}}))}{\|T(\overrightarrow{\boldsymbol{v}})\|_{\mathbf{H}}},
$$

which, due to the foregoing estimates, gives (3.16). On the other hand, introducing the operator $\widetilde{T}: \mathbf{H} \rightarrow \mathbf{H}$ as $\widetilde{T}(\overrightarrow{\boldsymbol{v}}):=\left(-\boldsymbol{v}_{\mathrm{B}}, \boldsymbol{z}_{\mathrm{B}},-\boldsymbol{v}_{\mathrm{D}}\right) \quad \forall \overrightarrow{\boldsymbol{v}}:=\left(\boldsymbol{v}_{\mathrm{B}}, \boldsymbol{z}_{\mathrm{B}}, \boldsymbol{v}_{\mathrm{D}}\right) \in \mathbf{H}$, we realize that $\|\widetilde{T}(\overrightarrow{\boldsymbol{v}})\|_{\mathbf{H}}=\|\overrightarrow{\boldsymbol{v}}\|_{\mathbf{H}}, \mathbf{a}(\overrightarrow{\boldsymbol{v}}, \overrightarrow{\boldsymbol{w}})=$ $\mathbf{a}(\widetilde{T}(\overrightarrow{\boldsymbol{w}}), \widetilde{T}(\overrightarrow{\boldsymbol{v}})) \quad \forall \overrightarrow{\boldsymbol{v}}, \overrightarrow{\boldsymbol{w}} \in \mathbf{H}, \widetilde{T}(\overrightarrow{\boldsymbol{v}}) \in \mathbf{V} \quad \forall \overrightarrow{\boldsymbol{v}} \in \mathbf{V}$, and $\widetilde{T}: \mathbf{V} \rightarrow \mathbf{V}$ is an isomorphism. It follows easily that

$$
\sup _{\substack{\overrightarrow{\boldsymbol{v}} \in \mathbf{V} \\ \boldsymbol{v} \neq \mathbf{0}}} \frac{\mathbf{a}(\overrightarrow{\boldsymbol{v}}, \overrightarrow{\boldsymbol{w}})}{\|\overrightarrow{\boldsymbol{v}}\|_{\mathbf{H}}}=\sup _{\substack{\overrightarrow{\boldsymbol{v}} \in \mathbf{V} \\ \boldsymbol{v} \neq \mathbf{0}}} \frac{\mathbf{a}(\widetilde{T}(\overrightarrow{\boldsymbol{w}}), \widetilde{T}(\overrightarrow{\boldsymbol{v}}))}{\|\widetilde{T}(\overrightarrow{\boldsymbol{v}})\|_{\mathbf{H}}}=\sup _{\substack{\overrightarrow{\boldsymbol{v}} \in \mathbf{V} \\ \overrightarrow{\boldsymbol{v}} \neq \mathbf{0}}} \frac{\mathbf{a}(\widetilde{T}(\overrightarrow{\boldsymbol{w}}), \overrightarrow{\boldsymbol{v}})}{\|\overrightarrow{\boldsymbol{v}}\|_{\mathbf{H}}},
$$

which, thanks to (3.16), yields (3.17) and completes the proof.

As a consequence of the previous analysis we can state the following main result. 
Theorem 3.2. Assume that $\mathbf{f}_{\mathrm{D}} \in \mathbf{L}^{2}\left(\Omega_{\mathrm{D}}\right)$ and $\mathbf{f}_{\mathrm{B}} \in \mathbf{L}^{2}\left(\Omega_{\mathrm{B}}\right)$. Then there exists a unique

$(\overrightarrow{\boldsymbol{u}}, \vec{p}):=\left(\left(\boldsymbol{u}_{\mathrm{B}}, \boldsymbol{\omega}_{\mathrm{B}}, \boldsymbol{u}_{\mathrm{D}}\right),\left(p_{\mathrm{B}}, p_{\mathrm{D}}, \lambda\right)\right) \in \mathbf{H} \times \mathbf{Q}_{0}$ solution of the modified mixed formulation (3.10). Moreover, there exists $C>0$ such that

$$
\|\overrightarrow{\boldsymbol{u}}\|_{\mathbf{H}}+\|\vec{p}\|_{\mathbf{Q}} \leq C\left\{\left\|\boldsymbol{f}_{\mathrm{D}}\right\|_{0, \Omega_{\mathrm{D}}}+\left\|\boldsymbol{f}_{\mathrm{B}}\right\|_{0, \Omega_{\mathrm{B}}}\right\}
$$

Proof. Thanks to Lemma 3.4 and 3.5, the proof is a straightforward application of the continuous BabuškaBrezzi theory. In particular, it is clear from the definition of $\mathbf{F}$ (cf. (2.14)) that $\|\mathbf{F}\|_{\mathbf{H}^{\prime}}$ is bounded by the right hand side of $(3.21)$.

We end this section by remarking that the way of proving the inf-sup conditions for the bilinear form a (cf. Lemma 3.5), namely using suitable operators $T$ and $\widetilde{T}$ to get a lower bound of the suprema involved, corresponds basically to what has been recently denominated in the literature as $T$-coercivity (see, e.g. [11], [15]). Nevertheless, the same idea, without any particular name of it, had been employed previously at least in the context of fluid-solid interaction problems (see, e.g. [21], [22], and [23]).

\section{The mixed finite element method}

In this section we introduce and analyze a mixed finite element scheme for (3.10). More precisely, we first define the associated Galerkin scheme and establish suitable assumptions on the finite element subspaces ensuring that it becomes well posed. Then, we provide specific examples satisfying the required hypotheses. In what follows, given an integer $k \geq 0$ and a subset $S$ of $\mathbb{R}^{3}$, we denote by $P_{k}(S)$ the space of polynomials in $S$ of total degree $\leq k$. In addition, according to the notation introduced in Section 1, we let $\mathbf{P}_{k}(S)=\left[P_{k}(S)\right]^{3}$.

\subsection{Preliminaries and main results}

We begin by selecting a set of arbitrary discrete spaces, namely

$$
\begin{aligned}
\mathbf{H}_{h}^{\mathrm{B}} \subseteq \mathbf{H}_{\mathrm{B}}\left(\operatorname{div} ; \Omega_{\mathrm{B}}\right), & \mathbf{H}_{0, h}^{\mathrm{B}} \subseteq \mathbf{H}_{0}\left(\mathbf{c u r l} ; \Omega_{\mathrm{B}}\right), \\
\mathrm{Q}_{h}^{\mathrm{B}} \subseteq \mathrm{L}^{2}\left(\Omega_{\mathrm{B}}\right), & \mathbf{H}_{h}^{\mathrm{D}} \subseteq \mathbf{H}_{\mathrm{D}}\left(\operatorname{div} ; \Omega_{\mathrm{D}}\right),
\end{aligned}
$$

In addition, in order to deal with the mean value condition for the Brinkman pressure $p_{\mathrm{B}}$, and also to handle the assumptions guaranteeing the discrete inf-sup condition for $\mathbf{b}$, we need to define

$$
\mathrm{Q}_{h, 0}^{\mathrm{B}}:=\mathrm{Q}_{h}^{\mathrm{B}} \cap \mathrm{L}_{0}^{2}\left(\Omega_{\mathrm{B}}\right) \quad \text { and } \quad \mathrm{Q}_{h, 0}^{\mathrm{D}}:=\mathrm{Q}_{h}^{\mathrm{D}} \cap \mathrm{L}_{0}^{2}\left(\Omega_{\mathrm{D}}\right) .
$$

Hence, setting the global spaces

$$
\mathbf{H}_{h}:=\mathbf{H}_{h}^{\mathrm{B}} \times \mathbf{H}_{0, h}^{\mathrm{B}} \times \mathbf{H}_{h}^{\mathrm{D}} \quad \text { and } \quad \mathbf{Q}_{0, h}:=\mathrm{Q}_{h, 0}^{\mathrm{B}} \times \mathrm{Q}_{h}^{\mathrm{D}} \times \mathrm{Q}_{h}^{\Sigma},
$$

the Galerkin scheme for (3.10) becomes: Find $\overrightarrow{\boldsymbol{u}}_{h}:=\left(\boldsymbol{u}_{h}^{\mathrm{B}}, \boldsymbol{\omega}_{h}^{\mathrm{B}}, \boldsymbol{u}_{h}^{\mathrm{D}}\right) \in \mathbf{H}_{h}$ and $\vec{p}_{h}:=\left(p_{h}^{\mathrm{B}}, p_{h}^{\mathrm{D}}, \lambda_{h}\right) \in \mathbf{Q}_{0, h}$ such that

$$
\begin{array}{rlr}
\mathbf{a}\left(\overrightarrow{\boldsymbol{u}}_{h}, \overrightarrow{\boldsymbol{v}}_{h}\right)+\mathbf{b}\left(\overrightarrow{\boldsymbol{v}}_{h}, \vec{p}_{h}\right) & =\mathbf{F}\left(\overrightarrow{\boldsymbol{v}}_{h}\right) \quad \forall \overrightarrow{\boldsymbol{v}}_{h}:=\left(\boldsymbol{v}_{h}^{\mathrm{B}}, \boldsymbol{z}_{h}^{\mathrm{B}}, \boldsymbol{v}_{h}^{\mathrm{D}}\right) \in \mathbf{H}_{h}, \\
\mathbf{b}\left(\overrightarrow{\boldsymbol{u}}_{h}, \vec{q}_{h}\right) & =\mathbf{G}\left(\vec{q}_{h}\right) & \forall \vec{q}_{h}:=\left(q_{h}^{\mathrm{B}}, q_{h}^{\mathrm{D}}, \xi_{h}\right) \in \mathbf{Q}_{0, h} .
\end{array}
$$

We now aim to derive general hypotheses on the finite element subspaces introduced in (4.1) ensuring, by means of the discrete Babuška-Brezzi theory, that the Galerkin scheme (4.4) becomes well-posed. Our approach consists of adapting to the present discrete case the arguments employed in Section 3 for the analysis of the continuous problem, mainly those from the proofs of Lemmas 3.4 and 3.5. We begin by observing that in order to have meaningful spaces $\mathrm{Q}_{h, 0}^{\mathrm{B}}$ and $\mathrm{Q}_{h, 0}^{\mathrm{D}}$ (cf. (4.2)), we need to be able to eliminate constants polynomials from $\mathrm{Q}_{h}^{\mathrm{B}}$ and $\mathrm{Q}_{h}^{\mathrm{D}}$. This request is certainly satisfied if we assume that:

(H.0) $P_{0}\left(\Omega_{\mathrm{B}}\right) \subseteq \mathrm{Q}_{h}^{\mathrm{B}}$ and $P_{0}\left(\Omega_{\mathrm{D}}\right) \subseteq \mathrm{Q}_{h}^{\mathrm{D}}$, 
which, in turn, yields the analogue orthogonal decompositions suggested by (1.1), that is

$$
\mathrm{Q}_{h}^{\mathrm{B}}=\mathrm{Q}_{h, 0}^{\mathrm{B}} \oplus P_{0}\left(\Omega_{\mathrm{B}}\right) \quad \text { and } \quad \mathrm{Q}_{h}^{\mathrm{D}}=\mathrm{Q}_{h, 0}^{\mathrm{D}} \oplus P_{0}\left(\Omega_{\mathrm{D}}\right) .
$$

Next, according to the same arguments utilized in the proof of Lemma 3.4, which actually are determined by those employed in the proofs of Lemmata 3.1, 3.2, and 3.3, we realize that in order to show the discrete inf-sup condition for $\mathbf{b}$ on $\mathbf{H}_{h} \times \mathbf{Q}_{0, h}$, we need to assume the following hypothesis:

(H.1) there holds $P_{0}(\Sigma) \subseteq \mathrm{Q}_{h}^{\Sigma}$ and there exist $\widetilde{\beta}_{\mathrm{B}}, \widetilde{\beta}_{\mathrm{D}}>0$, independent of $h$, such that

$$
S_{h}^{\mathrm{B}}\left(q_{h}^{\mathrm{B}}, \xi_{h}\right):=\sup _{\substack{\boldsymbol{v}_{h}^{\mathrm{B}} \in \mathbf{H}_{h}^{\mathrm{B}} \\ \boldsymbol{v}_{h}^{\mathrm{B}} \neq \mathbf{0}}} \frac{\int_{\Omega_{\mathrm{B}}} q_{h}^{\mathrm{B}} \operatorname{div} \boldsymbol{v}_{h}^{\mathrm{B}}-\left\langle\boldsymbol{v}_{h}^{\mathrm{B}} \cdot \boldsymbol{n}, \xi_{h}\right\rangle_{\Sigma}}{\left\|\boldsymbol{v}_{h}^{\mathrm{B}}\right\|_{\text {div } ; \Omega_{\mathrm{B}}}} \geq \widetilde{\beta}_{\mathrm{B}}\left\{\left\|q_{h}^{\mathrm{B}}\right\|_{0, \Omega_{\mathrm{B}}}+\left\|\xi_{h}\right\|_{1 / 2, \Sigma}\right\}
$$

for all $\left(q_{h}^{\mathrm{B}}, \xi_{h}\right) \in \mathrm{Q}_{h, 0}^{\mathrm{B}} \times \mathrm{Q}_{h}^{\Sigma}$, and

$$
S_{h}^{\mathrm{D}}\left(q_{h}^{\mathrm{D}}, \xi_{h}\right):=\sup _{\substack{\boldsymbol{v}_{h}^{\mathrm{D}} \in \mathbf{H}_{h}^{\mathrm{D}} \\ \boldsymbol{v}_{h}^{\mathrm{D}} \neq \mathbf{0}}} \frac{\int_{\Omega_{\mathrm{D}}} q_{h}^{\mathrm{D}} \operatorname{div} \boldsymbol{v}_{h}^{\mathrm{D}}+\left\langle\boldsymbol{v}_{h}^{\mathrm{D}} \cdot \boldsymbol{n}, \xi_{h}\right\rangle_{\Sigma}}{\left\|\boldsymbol{v}_{h}^{\mathrm{D}}\right\|_{\text {div } ; \Omega_{\mathrm{D}}}} \geq \widetilde{\beta}_{\mathrm{D}}\left\{\left\|q_{h}^{\mathrm{D}}\right\|_{0, \Omega_{\mathrm{D}}}+\left\|\xi_{h}\right\|_{1 / 2, \Sigma}\right\}
$$

for all $\left(q_{h}^{\mathrm{D}}, \xi_{h}\right) \in \mathrm{Q}_{h, 0}^{\mathrm{D}} \times \mathrm{Q}_{h}^{\Sigma}$.

On the other hand, we now look at the discrete kernel of $\mathbf{b}$, which is defined by

$$
\mathbf{V}_{h}:=\left\{\overrightarrow{\boldsymbol{v}}_{h}:=\left(\boldsymbol{v}_{h}^{\mathrm{B}}, \boldsymbol{z}_{h}^{\mathrm{B}}, \boldsymbol{v}_{h}^{\mathrm{D}}\right) \in \mathbf{H}_{h}: \quad \mathbf{b}\left(\overrightarrow{\boldsymbol{v}}_{h}, \vec{q}_{h}\right)=0 \quad \forall \vec{q}_{h}:=\left(q_{h}^{\mathrm{B}}, q_{h}^{\mathrm{D}}, \xi_{h}\right) \in \mathbf{Q}_{0, h}\right\} .
$$

Actually, in order to have a more explicit definition of $\mathbf{V}_{h}$, similarly as obtained for the continuous kernel $\mathbf{V}$ (cf. (3.13)), we now introduce the following assumption

(H.2) $\operatorname{div} \mathbf{H}_{h}^{\mathrm{B}} \subseteq \mathrm{Q}_{h}^{\mathrm{B}} \quad$ and $\operatorname{div} \mathbf{H}_{h}^{\mathrm{D}} \subseteq \mathrm{Q}_{h}^{\mathrm{D}}$,

which, together with (4.3) and (4.5), implies

$$
\mathbf{V}_{h}=\mathbf{V}_{\mathrm{B}, \mathrm{D}}^{h} \cap \mathbf{V}_{\Sigma}^{h},
$$

with

$$
\mathbf{V}_{\mathrm{B}, \mathrm{D}}^{h}:=\left\{\overrightarrow{\boldsymbol{v}}_{h}:=\left(\boldsymbol{v}_{h}^{\mathrm{B}}, \boldsymbol{z}_{h}^{\mathrm{B}}, \boldsymbol{v}_{h}^{\mathrm{D}}\right) \in \mathbf{H}_{h}: \quad \operatorname{div} \boldsymbol{v}_{h}^{\mathrm{B}} \in P_{0}\left(\Omega_{\mathrm{B}}\right) \quad \text { and } \quad \operatorname{div} \boldsymbol{v}_{h}^{\mathrm{D}}=0 \text { in } \Omega_{\mathrm{D}}\right\},
$$

and

$$
\mathbf{V}_{\Sigma}^{h}:=\left\{\overrightarrow{\boldsymbol{v}}_{h}:=\left(\boldsymbol{v}_{h}^{\mathrm{B}}, \boldsymbol{z}_{h}^{\mathrm{B}}, \boldsymbol{v}_{h}^{\mathrm{D}}\right) \in \mathbf{H}_{h}: \quad\left\langle\boldsymbol{v}_{h}^{\mathrm{B}} \cdot \boldsymbol{n}-\boldsymbol{v}_{h}^{\mathrm{D}} \cdot \boldsymbol{n}, \xi_{h}\right\rangle_{\Sigma}=0 \quad \forall \xi_{h} \in \mathrm{Q}_{h}^{\Sigma}\right\} .
$$

Since $\mathbf{V}_{\mathrm{B}, \mathrm{D}}^{h} \subseteq \mathbf{V}_{\mathrm{B}, \mathrm{D}}$ (cf. (3.14)), it is clear that inequality (3.19) is also valid in $\mathbf{V}_{\mathrm{B}, \mathrm{D}}^{h}$ and hence in the discrete kernel $\mathbf{V}_{h}$. Consequently, in order to show the discrete coerciveness of $\mathbf{a}$ on $\mathbf{V}_{h}$ by adapting the procedure utilized in the proof of Lemma 3.5, it just remains to assume the following hypothesis

(H.3) $\operatorname{curl} \mathbf{H}_{0, h}^{\mathrm{B}} \subseteq \mathbf{H}_{h}^{\mathrm{B}}$.

Having established hypotheses (H.0), (H.1), (H.2), and (H.3), we now reconfirm that they suffice to show that our Galerkin scheme (4.4) is well-posed and convergent. We begin with the discrete inf-sup condition for $\mathbf{b}$. 
Lemma 4.1. There exists $\widehat{\beta}>0$, independent of $h$, such that

$$
S_{h}\left(\vec{q}_{h}\right):=\sup _{\substack{\overrightarrow{\boldsymbol{v}}_{h} \in \mathbf{H}_{h} \\ \overrightarrow{\boldsymbol{v}}_{h} \neq \mathbf{0}}} \frac{\mathbf{b}\left(\overrightarrow{\boldsymbol{v}}_{h}, \vec{q}_{h}\right)}{\left\|\overrightarrow{\boldsymbol{v}}_{h}\right\|_{\mathbf{H}}} \geq \widehat{\beta}\left\|_{\vec{q}_{h}}\right\|_{\mathbf{Q}} \quad \forall \vec{q}_{h} \in \mathbf{Q}_{0, h} .
$$

Proof. Given $\vec{q}_{h}:=\left(q_{h}^{\mathrm{B}}, q_{h}^{\mathrm{D}}, \xi_{h}\right) \in \mathbf{Q}_{0, h}$, we let $q_{h, 0}^{\mathrm{D}} \in \mathrm{Q}_{h, 0}^{\mathrm{D}}$ and $c_{\mathrm{D}} \in \mathbb{R}$ such that $q_{h}^{\mathrm{D}}=q_{h, 0}^{\mathrm{D}}+c_{\mathrm{D}}$. Then, reasoning as in the proof of Lemma 3.3 , which in this case reduces to integrate by parts in $\Omega_{\mathrm{D}}$ only (since $q_{h}^{\mathrm{B}}$ is already in $\left.\mathrm{Q}_{h, 0}^{\mathrm{B}}\right)$, we find, using the notations from (H.1), that

$$
S_{h}\left(\vec{q}_{h}\right) \geq S_{h}^{\mathrm{B}}\left(q_{h}^{\mathrm{B}}, \xi_{h}\right) \quad \text { and } \quad S_{h}\left(\vec{q}_{h}\right) \geq S_{h}^{\mathrm{D}}\left(q_{h, 0}^{\mathrm{D}}, \xi_{h}-c_{\mathrm{D}}\right)
$$

In this way, since thanks to the first assumption in (H.1) we have that $\xi_{h}-c_{\mathrm{D}}$ belongs to $\mathrm{Q}_{h}^{\Sigma}$, the foregoing inequalities and a straightforward application of the discrete inf-sup conditions (4.6) and (4.7), imply

$$
S_{h}\left(\vec{q}_{h}\right) \geq \frac{1}{2}\left(\widetilde{\beta}_{\mathrm{B}}+\widetilde{\beta}_{\mathrm{D}}\right)\left\{\left\|q_{h}^{\mathrm{B}}\right\|_{0, \Omega_{\mathrm{B}}}+\left\|\xi_{h}\right\|_{1 / 2, \Sigma}+\left\|q_{h, 0}^{\mathrm{D}}\right\|_{0, \Omega_{\mathrm{D}}}+\left\|\xi_{h}-c_{\mathrm{D}}\right\|_{1 / 2, \Sigma}\right\} .
$$

The proof is concluded by employing the triangle inequality, exactly as we did for Lemma 3.4.

The discrete inf-sup condition for a on $\mathbf{V}_{h}$ is proved next. Since $\mathbf{V}_{h}$ is finite dimensional, it suffices to show one of the discrete analogues of the inequalities provided in Lemma 3.5.

Lemma 4.2. There exists $\widehat{\varrho}>0$, independent of $h$, such that

$$
\sup _{\substack{\overrightarrow{\boldsymbol{w}}_{h} \in \mathbf{V}_{h} \\ \overrightarrow{\boldsymbol{w}}_{h} \neq \mathbf{0}}} \frac{\mathbf{a}\left(\overrightarrow{\boldsymbol{v}}_{h}, \overrightarrow{\boldsymbol{w}}_{h}\right)}{\left\|\overrightarrow{\boldsymbol{w}}_{h}\right\|_{\mathbf{H}}} \geq \widehat{\varrho}\left\|\overrightarrow{\boldsymbol{v}}_{h}\right\|_{\mathbf{H}} \quad \forall \overrightarrow{\boldsymbol{v}}_{h} \in \mathbf{V}_{h}
$$

Proof. Given $\overrightarrow{\boldsymbol{v}}_{h}:=\left(\boldsymbol{v}_{h}^{\mathrm{B}}, \boldsymbol{z}_{h}^{\mathrm{B}}, \boldsymbol{v}_{h}^{\mathrm{D}}\right) \in \mathbf{V}_{h}$, we know from (3.19) that

$$
\mathbf{a}\left(\overrightarrow{\boldsymbol{v}}_{h}, \overrightarrow{\boldsymbol{v}}_{h}\right) \geq \widetilde{\varrho}_{1}\left\{\left\|\boldsymbol{v}_{h}^{\mathrm{B}}\right\|_{\mathrm{div} ; \Omega_{\mathrm{B}}}^{2}+\left\|\boldsymbol{z}_{h}^{\mathrm{B}}\right\|_{0, \Omega_{\mathrm{B}}}^{2}+\left\|\boldsymbol{v}_{h}^{\mathrm{D}}\right\|_{\mathrm{div} ; \Omega_{\mathrm{D}}}^{2}\right\}
$$

In addition, thanks to the result in [27, Chapter I, Section 2.3, Remark 2.5] and our assumption (H.3), we find that $\operatorname{curl} \boldsymbol{z}_{h}^{\mathrm{B}} \in \mathbf{H}_{0}\left(\operatorname{div} ; \Omega_{\mathrm{B}}\right) \cap \mathbf{H}_{h}^{\mathrm{B}}$, and hence $T_{0}\left(\overrightarrow{\boldsymbol{v}}_{h}\right):=\left(\mathbf{c u r l} \boldsymbol{z}_{h}^{\mathrm{B}}, \boldsymbol{z}_{h}^{\mathrm{B}}, \mathbf{0}\right)$ clearly belongs to $\mathbf{V}_{h}$ (cf. (4.9)). The rest of the proof proceeds as in Lemma 3.5. Moreover, it is easy to realize that the constants $c$ and $c_{0}$ defining now $T\left(\overrightarrow{\boldsymbol{v}}_{h}\right):=c \overrightarrow{\boldsymbol{v}}_{h}+c_{0} T_{0}\left(\overrightarrow{\boldsymbol{v}}_{h}\right)$ can be taken exactly as those chosen in the proof of that lemma, so that the resulting constant $\varrho$ of the present result coincides with $\widetilde{\varrho}$ in $(3.16)$ and $(3.17)$.

The following main result is a direct consequence of the previous analysis.

Theorem 4.1. Assume that $\mathbf{f}_{\mathrm{D}} \in \mathbf{L}^{2}\left(\Omega_{\mathrm{D}}\right)$ and $\mathbf{f}_{\mathrm{B}} \in \mathbf{L}^{2}\left(\Omega_{\mathrm{B}}\right)$. In addition, suppose that (H.0), (H.1), (H.2), and (H.3) hold. Then there exists a unique $\left(\overrightarrow{\boldsymbol{u}}_{h}, \vec{p}_{h}\right):=\left(\left(\boldsymbol{u}_{h}^{\mathrm{B}}, \boldsymbol{\omega}_{h}^{\mathrm{B}}, \boldsymbol{u}_{h}^{\mathrm{D}}\right),\left(p_{h}^{\mathrm{B}}, p_{h}^{\mathrm{D}}, \lambda_{h}\right)\right) \in \mathbf{H}_{h} \times \mathbf{Q}_{0, h}$ solution of the Galerkin scheme (4.4). Moreover, there exist $C_{1}, C_{2}>0$, independent of $h$, such that

$$
\left\|\overrightarrow{\boldsymbol{u}}_{h}\right\|_{\mathbf{H}}+\left\|\vec{p}_{h}\right\|_{\mathbf{Q}} \leq C_{1}\left\{\left\|\boldsymbol{f}_{\mathrm{D}}\right\|_{0, \Omega_{\mathrm{D}}}+\left\|\boldsymbol{f}_{\mathrm{B}}\right\|_{0, \Omega_{\mathrm{B}}}\right\}
$$

and

$$
\left\|(\overrightarrow{\boldsymbol{u}}, \vec{p})-\left(\overrightarrow{\boldsymbol{u}}_{h}, \vec{p}_{h}\right)\right\|_{\mathbf{H} \times \mathbf{Q}} \leq C_{2}\left\{\operatorname{dist}\left(\overrightarrow{\boldsymbol{u}}, \mathbf{H}_{h}\right)+\operatorname{dist}\left(\vec{p}, \mathbf{Q}_{0, h}\right)\right\}
$$

Proof. Thanks to Lemma 4.1 and 4.2, the proof results as a straightforward application of the discrete Babuška-Brezzi theory. 


\subsection{Specific finite element subspaces}

We now specify concrete examples of finite element subspaces satisfying the hypotheses introduced in the previous section. For this purpose, we now let $\mathcal{T}_{h}$ be a regular family of triangulations of $\bar{\Omega}_{\mathrm{B}} \cup \bar{\Omega}_{\mathrm{D}}$ by tetrahedra $K$ of diameter $h_{K}$ with mesh size $h:=\max \left\{h_{K}: \quad K \in \mathcal{T}_{h}\right\}$, such that $\mathcal{T}_{h}\left(\Omega_{\star}\right):=\left\{K \in \mathcal{T}_{h}: \quad K \subseteq \bar{\Omega}_{\star}\right\}$ is a triangulation of $\Omega_{\star}$ for each $\star \in\{\mathrm{B}, \mathrm{D}\}$. Then, we denote by $\mathcal{T}_{h}(\Sigma)$ the triangulation on $\Sigma$ induced by $\mathcal{T}_{h}$ (either from $\Omega_{\mathrm{B}}$ or $\Omega_{\mathrm{D}}$ ). Also, for reasons that will become clear below, we introduce an independent triangulation $\mathcal{T}_{\tilde{h}}(\Sigma)$ of $\Sigma$ by triangles $\widetilde{T}$ of diameter $h_{\widetilde{T}}$, and define $\tilde{h}:=\max \left\{h_{\widetilde{T}}: \quad \widetilde{T} \in \mathcal{T}_{\tilde{h}}(\Sigma)\right\}$.

\subsubsection{Definition of subspaces}

We first introduce the finite element subspaces

$$
\begin{aligned}
\mathbf{H}_{h}^{\star} & :=\left\{\boldsymbol{v}_{h}^{\star} \in \mathbf{H}_{\star}\left(\operatorname{div} ; \Omega_{\star}\right):\left.\quad \boldsymbol{v}_{h}^{\star}\right|_{K} \in \mathbb{R T}_{0}(K) \quad \forall K \in \mathcal{T}_{h}\left(\Omega_{\star}\right)\right\}, \\
\mathrm{Q}_{h}^{\star} & :=\left\{q_{h} \in \mathrm{L}^{2}\left(\Omega_{\star}\right):\left.\quad q_{h}\right|_{K} \in P_{0}(K) \quad \forall K \in \mathcal{T}_{h}\left(\Omega_{\star}\right)\right\}, \\
\mathrm{Q}_{h, 0}^{\star} & :=\mathrm{Q}_{h}^{\star} \cap \mathrm{L}_{0}^{2}\left(\Omega_{\star}\right),
\end{aligned}
$$

where $\star \in\{\mathrm{B}, \mathrm{D}\}$, and for any $K \in \mathcal{T}_{h}\left(\Omega_{\star}\right)$

$$
\mathbb{R T}_{0}(K):=\mathbf{P}_{0}(K) \oplus P_{0}(K) \boldsymbol{x}
$$

is the local Raviart-Thomas space of lowest order. In addition, we set

$$
\mathbf{H}_{0, h}^{\mathrm{B}}:=\left\{\boldsymbol{z}_{h}^{\mathrm{B}} \in \mathbf{H}_{0}\left(\mathbf{c u r l} ; \Omega_{\mathrm{B}}\right):\left.\quad \boldsymbol{z}_{h}^{\mathrm{B}}\right|_{K} \in \mathbb{N D}_{1}(K) \quad \forall K \in \mathcal{T}_{h}\left(\Omega_{\mathrm{B}}\right)\right\},
$$

where for any $K \in \mathcal{T}_{h}\left(\Omega_{\mathrm{B}}\right)$

$$
\mathbb{N D}_{1}(K):=\mathbf{P}_{0}(K) \oplus \mathbf{P}_{0}(K) \times \boldsymbol{x}
$$

is the local edge space of Nédélec, that is

$$
\mathbb{N D}_{1}(K):=\left\{w: K \rightarrow \mathbb{C}^{3}: \quad w(\boldsymbol{x})=\boldsymbol{a}+\boldsymbol{b} \times \boldsymbol{x} \quad \forall \boldsymbol{x} \in K, \boldsymbol{a}, \boldsymbol{b} \in \mathbb{C}^{3}\right\} .
$$

Finally for the interface $\Sigma$ we consider the finite element subspace

$$
\mathrm{Q}_{\tilde{h}}^{\Sigma}:=\left\{\lambda_{\tilde{h}} \in \mathcal{C}^{0}(\Sigma):\left.\quad \lambda_{\tilde{h}}\right|_{\tilde{T}} \in P_{1}(\tilde{T}) \quad \forall \tilde{T} \in \mathcal{T}_{\tilde{h}}(\Sigma)\right\} .
$$

It is easy to check that these subspaces satisfy the hypotheses (H.0), (H.2) and (H.3).

On the other hand, for purposes of the analysis, we also need to define

$$
\Phi_{h}(\Sigma):=\left\{\psi_{h} \in \mathrm{L}^{2}(\Sigma):\left.\quad \psi_{h}\right|_{T} \in P_{0}(T) \quad \forall T \in \mathcal{T}_{h}(\Sigma)\right\}
$$

\subsubsection{Approximation properties}

In what follows $\star$ is a mute symbol taken in $\{\mathrm{B}, \mathrm{D}\}$. We let $\Pi_{h}^{\star}: \mathbf{H}^{1}\left(\Omega_{\star}\right) \rightarrow \mathbf{H}_{h}^{\star}$ be the usual RaviartThomas interpolation operator, that is, given a sufficiently smooth vector field $\boldsymbol{v}: \Omega_{\star} \rightarrow \mathbb{R}^{3}$, we define $\Pi_{h}^{\star}(\boldsymbol{v})$ as the only element of $\mathbf{H}_{h}^{\star}$ such that

$$
\int_{F} \Pi_{h}^{\star}(\boldsymbol{v}) \cdot \boldsymbol{n}=\int_{F} \boldsymbol{v} \cdot \boldsymbol{n} \quad \forall F \in \mathcal{E}_{h}^{\star}
$$

where $\mathcal{E}_{h}^{\star}$ is the set of faces of the triangulation $\mathcal{T}_{h}\left(\Omega_{\star}\right)$. We now recall some properties of $\Pi_{h}^{\star}$ and its local counterparts $\Pi_{K}^{\star}$ for each $K \in \mathcal{T}_{h}\left(\Omega_{\star}\right)$ (see, e.g [20]):

(a) $\Pi_{h}^{\star}$ is well defined in $\mathbf{H}^{\delta}\left(\Omega_{\star}\right) \cap \mathbf{H}\left(\operatorname{div} ; \Omega_{\star}\right)$ for any $\delta \in(0,1)$. 
(b) There holds $\operatorname{div} \Pi_{h}^{\star}(\boldsymbol{v})=\mathcal{P}_{h}^{\star}(\operatorname{div} \boldsymbol{v})$, where $\mathcal{P}_{h}^{\star}: \mathrm{L}^{2}\left(\Omega_{\star}\right) \rightarrow \mathrm{Q}_{h}^{\star}$ is the orthogonal projector. Equivalently

$$
\int_{\Omega_{\star}} q_{h} \operatorname{div} \Pi_{h}^{\star}(\boldsymbol{v})=\int_{\Omega_{\star}} q_{h} \operatorname{div}(\boldsymbol{v}) \quad \forall q_{h} \in \mathrm{Q}_{h}^{\star} .
$$

(c) For each face $F$ of $K$ there holds $\Pi_{K}^{\star}(\boldsymbol{v}) \cdot \boldsymbol{n}_{F}=\mathcal{P}_{F}\left(\boldsymbol{v} \cdot \boldsymbol{n}_{F}\right)$, where $\boldsymbol{n}_{F}$ is the unit outward normal on $F$ and $\mathcal{P}_{F}: \mathrm{L}^{2}(F) \rightarrow P_{0}(F)$ is the orthogonal projector.

(d) Given $\delta \in(0,1)$ and $\boldsymbol{v} \in \mathbf{H}^{\delta}\left(\Omega_{\star}\right) \cap \mathbf{H}\left(\right.$ div $\left.; \Omega_{\star}\right)$, there holds

$$
\left\|\boldsymbol{v}-\Pi_{K}^{\star}(\boldsymbol{v})\right\|_{0, K} \leq C h_{K}^{\delta}\left\{|\boldsymbol{v}|_{\delta, K}+\|\operatorname{div}(\boldsymbol{v})\|_{0, K}\right\} \quad \forall K \in \mathcal{T}_{h}\left(\Omega_{\star}\right) .
$$

Next, for any $\epsilon>0$ we introduce the Sobolev space

$$
\mathbf{H}^{\epsilon}\left(\operatorname{curl} ; \Omega_{\mathrm{B}}\right):=\left\{\boldsymbol{v} \in \mathbf{H}^{\epsilon}\left(\Omega_{\mathrm{B}}\right): \quad \operatorname{curl} \boldsymbol{v} \in \mathbf{H}^{\epsilon}\left(\Omega_{\mathrm{B}}\right)\right\},
$$

and endow it with its Hilbertian norm

$$
\|\boldsymbol{v}\|_{\mathbf{H}^{\epsilon}\left(\operatorname{curl} ; \Omega_{\mathrm{B}}\right)}:=\left\{\|\boldsymbol{v}\|_{\epsilon, \Omega_{\mathrm{B}}}^{2}+\|\operatorname{curl}(\boldsymbol{v})\|_{\epsilon, \Omega_{\mathrm{B}}}^{2}\right\}^{1 / 2} .
$$

Then for each face $F$ of $\mathcal{T}_{h}\left(\Omega_{\mathrm{B}}\right)$ we let $\boldsymbol{t}_{F}$ be a unit tangential vector on $F$. It follows from [3, Lemma 4.7] that if $\epsilon>1 / 2$ the interpolation operator $\Pi_{h}: \mathbf{H}^{\epsilon}\left(\mathbf{c u r l} ; \Omega_{\mathrm{B}}\right) \rightarrow \mathbf{H}_{0, h}^{\mathrm{B}}$ associated with the face finite element, which is characterized by

$$
\int_{F} \Pi_{h}(\boldsymbol{v}) \cdot \boldsymbol{t}_{F}=\int_{F} \boldsymbol{v} \cdot \boldsymbol{t}_{F} \quad \forall \text { faces } F \text { of } \mathcal{T}_{h}\left(\Omega_{\mathrm{B}}\right),
$$

is well defined and uniformly bounded. In addition, the following property of $\Pi_{h}$ holds.

Lemma 4.3. There exists $C>0$, independent of $h$, such that

$$
\left\|\boldsymbol{v}-\Pi_{h}(\boldsymbol{v})\right\|_{\mathbf{c u r l} ; \Omega_{\mathrm{B}}} \leq C h^{\epsilon}\|\boldsymbol{v}\|_{\mathbf{H}^{\epsilon}\left(\mathbf{c u r l} ; \Omega_{\mathrm{B}}\right)}
$$

for all $\boldsymbol{v} \in \mathbf{H}^{\epsilon}\left(\mathbf{c u r l} ; \Omega_{\mathrm{B}}\right)$ and for all $\epsilon \in(1 / 2,1]$.

Proof. See, [2, Proposition 5.6].

The approximation properties of the finite element subspaces involved are then established as follows (see, e.g $[13,31])$ :

$\left(\operatorname{AP}_{h}^{\boldsymbol{u}_{\star}}\right)$ there exists $C>0$, independent of $h$, such that for each $\delta \in(0,1]$ and for each $\boldsymbol{v} \in \mathbf{H}^{\delta}\left(\Omega_{\star}\right)$, with $\operatorname{div}(\boldsymbol{v}) \in \mathbf{H}^{\delta}\left(\Omega_{\star}\right)$, there holds

$$
\left\|\boldsymbol{v}-\Pi_{h}^{\star}(\boldsymbol{v})\right\|_{\operatorname{div} ; \Omega_{\star}} \leq C h^{\delta}\left\{\|\boldsymbol{v}\|_{\delta, \Omega_{\star}}+\|\operatorname{div}(\boldsymbol{v})\|_{\delta, \Omega_{\star}}\right\} \quad(\star \in\{\mathrm{B}, \mathrm{D}\}) .
$$

$\left(\operatorname{AP}_{h}^{p_{\star}}\right)$ there exists $C>0$, independent of $h$, such that for each $\delta \in(0,1]$ and for each $q \in \mathrm{H}^{\delta}\left(\Omega_{\star}\right)$, there holds

$$
\left\|q-\mathcal{P}_{h}^{\star}(q)\right\|_{0, \Omega_{\star}} \leq C h^{\delta}\|q\|_{\delta, \Omega_{\star}} \quad(\star \in\{\mathrm{B}, \mathrm{D}\}) .
$$

$\left(\mathrm{AP}_{h}^{\boldsymbol{\omega}_{\mathrm{B}}}\right)$ there exists $C>0$, independent of $h$, such that for each $\delta \in(1 / 2,1]$ and for each $\boldsymbol{z}_{\mathrm{B}} \in$ $\mathbf{H}^{\delta}\left(\operatorname{curl} ; \Omega_{\mathrm{B}}\right)$, there holds

$$
\left\|\boldsymbol{z}_{\mathrm{B}}-\Pi_{h}\left(\boldsymbol{z}_{\mathrm{B}}\right)\right\|_{\mathbf{c u r l} ; \Omega_{\mathrm{B}}} \leq C h^{\delta}\left\|\boldsymbol{z}_{\mathrm{B}}\right\|_{\mathbf{H}^{\delta}\left(\mathbf{c u r l} ; \Omega_{\mathrm{B}}\right)} .
$$

$\left(\operatorname{AP}_{\tilde{h}}^{\lambda}\right)$ there exists $C>0$, independent of $\tilde{h}$, such that for each $\delta \in(0,1]$ and for each $\xi \in \mathrm{H}^{1 / 2+\delta}(\Sigma)$, there holds

$$
\left\|\xi-\mathcal{P}_{\tilde{h}}(\xi)\right\|_{1 / 2, \Sigma} \leq C \tilde{h}^{\delta}\|\xi\|_{1 / 2+\delta, \Sigma},
$$

where $\mathcal{P}_{\tilde{h}}: \mathrm{H}^{1 / 2}(\Sigma) \rightarrow \mathrm{Q}_{\tilde{h}}^{\Sigma}$ is the orthogonal projector.

$\left(\mathrm{AP}_{h}{ }^{\psi}\right)$ there exists $C>0$, independent of $h$, such that for each $\delta \in(0,1]$ and for each $\varphi \in \mathrm{H}^{-1 / 2+\delta}(\Sigma)$, there holds

$$
\left\|\varphi-\mathcal{P}_{h}^{-1 / 2}(\varphi)\right\|_{-1 / 2, \Sigma} \leq C h_{\Sigma}^{\delta}\|\varphi\|_{-1 / 2+\delta, \Sigma}
$$

where $\mathcal{P}_{h}^{-1 / 2}: \mathrm{H}^{-1 / 2}(\Sigma) \rightarrow \Phi_{h}(\Sigma)$ is the orthogonal projector. 


\subsubsection{Stable discrete liftings}

In this section, as usual we let $\star$ be a mute symbol taken in $\{\mathrm{B}, \mathrm{D}\}$, and provide sufficient conditions for the existence of a stable discrete lifting $\mathcal{L}_{h}: \Phi_{h}(\Sigma) \rightarrow \mathbf{H}_{h}^{\star}$. To this end, we proceed as in [20, Theorem 4.1], and assume first that $\mathcal{T}_{h}\left(\Omega_{\star}\right)$ is quasi-uniform in a neighborhood of $\Sigma$. This means that there exists a neighborhood of $\Sigma$, say $\Omega_{\Sigma}$, and a constant $c>0$, independent of $h$, such that, denoting

$$
\mathcal{T}_{h, \Sigma}^{\star}:=\left\{K \in \mathcal{T}_{h}\left(\Omega_{\star}\right): \quad K \cap \Omega_{\Sigma} \neq \emptyset\right\}
$$

there holds

$$
\max _{K \in \mathcal{T}_{h, \Sigma}^{\star}} h_{K} \leq c \min _{K \in \mathcal{T}_{h, \Sigma}^{\star}} h_{K}
$$

Now, because of the regularity of $\mathcal{T}_{h}\left(\Omega_{\star}\right)$, the quasi-uniformity assumption around $\Sigma$ implies that the partition $\mathcal{T}_{h}(\Sigma)$ inherited from $\mathcal{T}_{h}\left(\Omega_{\star}\right)$ is quasi-uniform as well, which implies that $\Phi_{h}(\Sigma)$ satisfies the inverse inequality (see, [20, Lemma 4.6])

$$
\left\|\psi_{h}\right\|_{-1 / 2+\delta, \Sigma} \leq C h_{\Sigma}^{-\delta}\left\|\psi_{h}\right\|_{-1 / 2, \Sigma} \quad \forall \psi_{h} \in \Phi_{h}(\Sigma), \quad \forall \delta \in[0,1 / 2],
$$

where $h_{\Sigma}:=\max \left\{h_{T}: \quad T \in \mathcal{T}_{h}(\Sigma)\right\}$

Lemma 4.4. There exist a linear operator $\mathcal{L}_{h}: \Phi_{h}(\Sigma) \rightarrow \mathbf{H}_{h}^{\star}$ and a constant $C_{\mathcal{L}}>0$, independent of $h$, such that for each $\psi_{h} \in \Phi_{h}(\Sigma)$ there hold

$$
\mathcal{L}_{h}\left(\psi_{h}\right) \cdot \boldsymbol{n}=\psi_{h} \quad \text { on } \quad \Sigma, \quad\left\|\mathcal{L}_{h}\left(\psi_{h}\right)\right\|_{\operatorname{div}, \Omega_{\star}} \leq C_{\mathcal{L}}\left\|\psi_{h}\right\|_{-1 / 2, \Sigma}, \quad \text { and } \quad \operatorname{div} \mathcal{L}_{h}\left(\psi_{h}\right) \in P_{0}\left(\Omega_{\star}\right) .
$$

Proof. Let $\psi_{h} \in \Phi_{h}(\Sigma)$, and let $z \in \mathrm{H}^{1}\left(\Omega_{\star}\right)$ be the unique solution of the boundary value problem

$$
\Delta z=\frac{1}{\left|\Omega_{\star}\right|}\left\langle\psi_{h}, 1\right\rangle_{\Sigma} \quad \text { in } \quad \Omega_{\star}, \quad \nabla z \cdot \boldsymbol{n}=\left\{\begin{array}{ccc}
\psi_{h} & \text { on } & \Sigma \\
0 & \text { on } & \Gamma_{\star}
\end{array}, \quad \int_{\Omega_{\star}} z=0 .\right.
$$

The corresponding continuous dependence result says that $\|z\|_{1, \Omega_{\star}} \leq c_{1}\left\|\psi_{h}\right\|_{-1 / 2, \Sigma}$. In turn, the elliptic regularity result (cf. [28]) establishes that there exists $\delta>0$ such that

$$
\|z\|_{1+\delta, \Omega_{\star}} \leq c_{2}\left\|\psi_{h}\right\|_{-1 / 2+\delta, \Sigma}
$$

In addition, notice that

$$
\operatorname{div}(\nabla z)=\Delta z=\frac{1}{\left|\Omega_{\star}\right|}\left\langle\psi_{h}, 1\right\rangle_{\Sigma} \in \mathbb{R} \quad \text { in } \quad \Omega_{\star} .
$$

It follows that $\nabla z \in \mathbf{H}^{\delta}\left(\Omega_{\star}\right) \cap \mathbf{H}\left(\operatorname{div} ; \Omega_{\star}\right)$, and hence we can define

$$
\mathcal{L}_{h}\left(\psi_{h}\right):=\Pi_{h}^{\star}(\nabla z)
$$

Next, from properties (b) and (c) of the Raviart-Thomas interpolation operator, we find that

$$
\operatorname{div} \mathcal{L}_{h}\left(\psi_{h}\right)=\operatorname{div} \Pi_{h}^{\star}(\nabla z)=\mathcal{P}_{h}^{\star}(\operatorname{div} \nabla z)=\mathcal{P}_{h}^{\star}(\Delta z)=\frac{1}{\left|\Omega_{\star}\right|}\left\langle\psi_{h}, 1\right\rangle_{\Sigma} \quad \text { in } \quad \Omega_{\star}
$$

and

$$
\mathcal{L}_{h}\left(\psi_{h}\right) \cdot \boldsymbol{n}=\Pi_{h}^{\star}(\nabla z) \cdot \boldsymbol{n}=\mathcal{P}_{h, \Sigma}(\nabla z \cdot \boldsymbol{n})=\mathcal{P}_{h, \Sigma}\left(\psi_{h}\right)=\psi_{h} \quad \text { on } \quad \Sigma,
$$

where $\mathcal{P}_{h, \Sigma}: \mathrm{L}^{2}(\Sigma) \rightarrow \Phi_{h}(\Sigma)$ is the orthogonal projector. It remains to show that $\mathcal{L}_{h}$ is uniformly bounded. To this end, we first observe that

$$
\left\|\mathcal{L}_{h}\left(\psi_{h}\right)\right\|_{\text {div }, \Omega_{\star}}^{2}=\left\|\mathcal{L}_{h}\left(\psi_{h}\right)\right\|_{0, \Omega_{\star}}^{2}+\left\|\frac{1}{\left|\Omega_{\star}\right|}\left\langle\psi_{h}, 1\right\rangle_{\Sigma}\right\|_{0, \Omega_{\star}}^{2} \leq\left\|\mathcal{L}_{h}\left(\psi_{h}\right)\right\|_{0, \Omega_{\star}}^{2}+c_{3}\left\|\psi_{h}\right\|_{-1 / 2, \Sigma}^{2} .
$$

Now, we divide $\Omega_{\star}$ into two regions

$$
\Omega_{\star, h}^{1}:=\cup\left\{K \in \mathcal{T}_{h}\left(\Omega_{\star}\right): \quad K \notin \mathcal{T}_{h, \Sigma}^{\star}\right\} \subseteq \Omega_{\star} \backslash \Omega_{\Sigma}, \quad \Omega_{\star, h}^{2}:=\Omega_{\star} \backslash \Omega_{\star, h}^{1}=\cup\left\{K \in \mathcal{T}_{h, \Sigma}^{\star}\right\},
$$


where we recall that $\mathcal{T}_{h, \Sigma}^{\star}:=\left\{K \in \mathcal{T}_{h}\left(\Omega_{\star}\right): \quad K \cap \Omega_{\Sigma} \neq \emptyset\right\}$. Then, since $\Omega_{\star} \backslash \Omega_{\Sigma}$ is strictly contained in $\Omega_{\star}$, the interior elliptic regularity result [30, Theorem 4.16] implies that $\left.z\right|_{\Omega_{\star} \backslash \Omega_{\Sigma}} \in \mathrm{H}^{2}\left(\Omega_{\star} \backslash \Omega_{\Sigma}\right)$ and

$$
\|z\|_{2, \Omega_{\star} \backslash \Omega_{\Sigma}} \leq c_{4}\left\|\psi_{h}\right\|_{-1 / 2, \Sigma} .
$$

It follows that

$$
\begin{aligned}
\left\|\mathcal{L}_{h}\left(\psi_{h}\right)\right\|_{0, \Omega_{\star}} & \leq\left\|\mathcal{L}_{h}\left(\psi_{h}\right)\right\|_{0, \Omega_{\star, h}^{1}}+\left\|\mathcal{L}_{h}\left(\psi_{h}\right)\right\|_{0, \Omega_{\star, h}^{2}} \\
& =\left\|\Pi_{h}^{\star}(\nabla z)\right\|_{0, \Omega_{\star, h}^{1}}+\left\|\Pi_{h}^{\star}(\nabla z)\right\|_{0, \Omega_{\star, h}^{2}} \\
& \leq c_{5}\|\nabla z\|_{1, \Omega_{\star, h}^{1}}+\|\nabla z\|_{0, \Omega_{\star, h}^{2}}+\left\|\nabla z-\Pi_{h}^{\star}(\nabla z)\right\|_{0, \Omega_{\star, h}^{2}} \\
& \leq c_{5}\|z\|_{2, \Omega_{\star, h}^{1}}+\|z\|_{1, \Omega_{\star, h}^{2}}+\left\|\nabla z-\Pi_{h}^{\star}(\nabla z)\right\|_{0, \Omega_{\star, h}^{2}} \\
& \leq c_{5} c_{4}\left\|\psi_{h}\right\|_{-1 / 2, \Sigma}+c_{1}\left\|\psi_{h}\right\|_{-1 / 2, \Sigma}+\left\|\nabla z-\Pi_{h}^{\star}(\nabla z)\right\|_{0, \Omega_{\star, h}^{2}} .
\end{aligned}
$$

On the other hand, applying estimate (4.17) and inverse inequality (4.20), we obtain that

$$
\begin{aligned}
\| \nabla z- & \Pi_{h}^{\star}(\nabla z)\left\|_{0, \Omega_{\star, h}^{2}}^{2}=\sum_{K \in \mathcal{T}_{h, \Sigma}^{\star}}\right\| \nabla z-\Pi_{K}^{\star}(\nabla z) \|_{0, K}^{2} \\
\leq & c_{6} \sum_{K \in \mathcal{T}_{h, \Sigma}^{\star}} h_{K}^{2 \delta}\left\{|\nabla z|_{\delta, K}^{2}+\left\|\frac{1}{\left|\Omega_{\star}\right|}\left\langle\psi_{h}, 1\right\rangle_{\Sigma}\right\|_{0, K}^{2}\right\} \\
\leq & c_{7} \max _{K \in \mathcal{T}_{h, \Sigma}^{\star}} h_{K}^{2 \delta}\left\{\|z\|_{1+\delta, \Omega_{\star, h}^{2}}^{2}+\left\|\psi_{h}\right\|_{-1 / 2, \Sigma}^{2}\right\} \\
\leq & c_{7} \max _{K \in \mathcal{T}_{h, \Sigma}^{\star}} h_{K}^{2 \delta}\left\{\|z\|_{1+\delta, \Omega_{\star}}^{2}+\left\|\psi_{h}\right\|_{-1 / 2, \Sigma}^{2}\right\} \\
\leq & c_{8} \max _{K \in \mathcal{T}_{h, \Sigma}^{\star}} h_{K}^{2 \delta}\left\{\left\|\psi_{h}\right\|_{-1 / 2+\delta, \Sigma}^{2}+\left\|\psi_{h}\right\|_{-1 / 2, \Sigma}^{2}\right\} \\
\leq & c_{8} \max _{K \in \mathcal{T}_{h, \Sigma}^{\star}} h_{K}^{2 \delta}\left\{h_{\Sigma}^{-2 \delta}\left\|\psi_{h}\right\|_{-1 / 2, \Sigma}^{2}+\left\|\psi_{h}\right\|_{-1 / 2, \Sigma}^{2}\right\} \\
\leq & c_{9}\left\|\psi_{h}\right\|_{-1 / 2, \Sigma}^{2},
\end{aligned}
$$

where the fact that $h_{K} \leq c h_{\Sigma} \quad \forall K \in \mathcal{T}_{h, \Sigma}^{\star}$ has been used in the last inequality. In this way, from (4.23), (4.24) and (4.25) we conclude that

$$
\left\|\mathcal{L}_{h}\left(\psi_{h}\right)\right\|_{\text {div }, \Omega_{\star}} \leq C_{\mathcal{L}}\left\|\psi_{h}\right\|_{-1 / 2, \Sigma} \quad \forall \psi_{h} \in \Phi_{h}(\Sigma),
$$

which, together with the identities (4.21) and (4.22), complete the proof.

We remark at this point that the quasi-uniformity assumption of $\mathcal{T}_{h}\left(\Omega_{*}\right)$ around $\Sigma$, which is needed here for the stable discrete lifting provided by Lemma 4.4, has been removed recently in [1, Theorem 2.1] for the case of locally refined meshes, when the lifting is from the whole boundary of the given domain. However, it is not clear from the analysis in [1] whether that result is also valid for a discrete lifting from part of the boundary (as it is required in the present case).

We now assume that the family of independent triangulations $\mathcal{T}_{\tilde{h}}(\Sigma)$ is also quasi-uniform, which implies that $\mathrm{Q}_{\tilde{h}}^{\Sigma}$ satisfies the inverse inequality, that is there exists a constant $C>0$, independent of $\tilde{h}$, such that for each $\delta \in[0,1)$ there holds (cf. [21, Lemma 7.4])

$$
\|\xi\|_{1 / 2+\delta, \Sigma} \leq C \tilde{h}^{-\delta}\|\xi\|_{1 / 2, \Sigma} \quad \forall \xi \in \mathrm{Q}_{\tilde{h}}^{\Sigma} .
$$

Then, we have the following result.

Lemma 4.5. There exist $C_{0}, \beta>0$, independent of $h_{\Sigma}$ and $\tilde{h}$, such that for all $h_{\Sigma} \leq C_{0} \tilde{h}$ there holds

$$
\sup _{\substack{\psi_{h} \in \Phi_{h}(\Sigma) \\ \psi_{h} \neq 0}} \frac{\left\langle\psi_{h}, \xi_{\tilde{h}}\right\rangle_{\Sigma}}{\left\|\psi_{h}\right\|_{-1 / 2, \Sigma}} \geq \beta\left\|\xi_{\tilde{h}}\right\|_{1 / 2, \Sigma} \quad \forall \xi_{\tilde{h}} \in \mathrm{Q}_{\tilde{h}}^{\Sigma}
$$


Proof. We proceed similarly as in [20, Lemma 4.11]. In fact, given $\xi_{\tilde{h}} \in \mathrm{Q}_{\tilde{h}}^{\Sigma} \backslash\{0\}$, we let $z \in \mathrm{H}^{1}\left(\Omega_{\star}\right)$ be the unique solution of the boundary value problem with mixed boundary conditions:

$$
-\Delta z+z=0 \quad \text { in } \quad \Omega_{\star}, \quad z=\xi_{\tilde{h}} \quad \text { on } \quad \Sigma, \quad \nabla z \cdot \boldsymbol{n}=0 \quad \text { on } \quad \Gamma_{\star} .
$$

Notice that the corresponding continuous dependence result gives

$$
\|z\|_{1, \Omega_{\star}} \leq C_{1}\left\|\xi_{\tilde{h}}\right\|_{1 / 2, \Sigma}
$$

and thanks to the trace theorem and a simple integration by parts procedure, we also have that

$$
C_{2}\left\|\xi_{\tilde{h}}\right\|_{1 / 2, \Sigma}^{2} \leq\|z\|_{1, \Omega_{\star}}^{2}=\left\langle\nabla z \cdot \boldsymbol{n}, \xi_{\tilde{h}}\right\rangle_{\Sigma} .
$$

On the other hand, since $\mathrm{Q}_{\tilde{h}}^{\Sigma} \subset \mathrm{H}^{1}(\Sigma)$, we obtain that $z \in \mathrm{H}^{1+\delta}\left(\Omega_{\star}\right)$ for some $\delta>0$ (see [28]), and there holds

$$
\|\nabla z \cdot \boldsymbol{n}\|_{-1 / 2+\delta, \Sigma} \leq C_{3}\|z\|_{1+\delta, \Omega_{\star}} \leq C_{4}\left\|\xi_{\tilde{h}}\right\|_{1 / 2+\delta, \Sigma} .
$$

We now let $\psi_{h}^{*}:=\mathcal{P}_{h}^{-1 / 2}(\nabla z \cdot \boldsymbol{n}) \in \Phi_{h}(\Sigma)$. Then, applying the approximation property $\left(\operatorname{AP}_{h}^{\psi}\right)$, the regularity estimate (4.30), and the inverse inequality (4.26), we deduce that

$$
\left\|\nabla z \cdot \boldsymbol{n}-\psi_{h}^{*}\right\|_{-1 / 2, \Sigma} \leq C_{5} h_{\Sigma}^{\delta}\|\nabla z \cdot \boldsymbol{n}\|_{-1 / 2+\delta, \Sigma} \leq C_{6} h_{\Sigma}^{\delta}\left\|\xi_{\tilde{h}}\right\|_{1 / 2+\delta, \Sigma} \leq C_{7}\left(\frac{h_{\Sigma}}{\tilde{h}}\right)^{\delta}\left\|\xi_{\tilde{h}}\right\|_{1 / 2, \Sigma} .
$$

Next, using that $\|\nabla z\|_{\operatorname{div}, \Omega_{\star}}=\|z\|_{1, \Omega_{\star}}$, it follows that

$$
\left\|\psi_{h}^{*}\right\|_{-1 / 2, \Sigma}=\left\|\mathcal{P}_{h}^{-1 / 2}(\nabla z \cdot \boldsymbol{n})\right\|_{-1 / 2, \Sigma} \leq\|\nabla z \cdot \boldsymbol{n}\|_{-1 / 2, \Sigma} \leq\|\nabla z\|_{\text {div }, \Omega_{\star}}=\|z\|_{1, \Omega_{\star}},
$$

which together with the estimate (4.28), imply

$$
\left\|\psi_{h}^{*}\right\|_{-1 / 2, \Sigma} \leq C_{8}\left\|\xi_{\tilde{h}}\right\|_{1 / 2, \Sigma} .
$$

Now, using (4.29) and the foregoing estimates, we find that

$$
\begin{aligned}
\left\langle\psi_{h}^{*}, \xi_{\tilde{h}}\right\rangle_{\Sigma} & =\left\langle\nabla z \cdot \boldsymbol{n}, \xi_{\tilde{h}}\right\rangle_{\Sigma}-\left\langle\nabla z \cdot \boldsymbol{n}-\psi_{h}^{*}, \xi_{\tilde{h}}\right\rangle_{\Sigma} \\
& \geq\left\{C_{2}-C_{7}\left(\frac{h_{\Sigma}}{\tilde{h}}\right)^{\delta}\right\}\left\|\xi_{\tilde{h}}\right\|_{1 / 2, \Sigma}^{2} \\
& \geq\left\{\frac{C_{2}}{C_{8}}-\frac{C_{7}}{C_{8}}\left(\frac{h_{\Sigma}}{\tilde{h}}\right)^{\delta}\right\}\left\|\xi_{\tilde{h}}\right\|_{1 / 2, \Sigma}\left\|\psi_{h}^{*}\right\|_{-1 / 2, \Sigma} .
\end{aligned}
$$

Consequently, we can write

$$
\sup _{\substack{\psi_{h} \in \Phi_{h}(\Sigma) \\ \psi_{h} \neq 0}} \frac{\left\langle\psi_{h}, \xi_{\tilde{h}}\right\rangle_{\Sigma}}{\left\|\psi_{h}\right\|_{-1 / 2, \Sigma}} \geq \frac{\left\langle\psi_{h}^{*}, \xi_{\tilde{h}}\right\rangle_{\Sigma}}{\left\|\psi_{h}^{*}\right\|_{-1 / 2, \Sigma}} \geq\left\{\frac{C_{2}}{C_{8}}-\frac{C_{7}}{C_{8}}\left(\frac{h_{\Sigma}}{\tilde{h}}\right)^{\delta}\right\}\left\|\xi_{\tilde{h}}\right\|_{1 / 2, \Sigma},
$$

from which, taking $h_{\Sigma} \leq C_{0} \tilde{h}$ with $C_{0}:=\left(\frac{C_{2}}{2 C_{7}}\right)^{1 / \delta}$, we conclude the proof.

\subsubsection{Verification of the discrete inf-sup conditions}

We are now in a position to prove the discrete inf-sup conditions required by hypotheses (H.1). To this end, we assume from now on that $\mathcal{T}_{h}\left(\Omega_{\mathrm{D}}\right)$ and $\mathcal{T}_{h}\left(\Omega_{\mathrm{B}}\right)$ are quasi-uniform in a neighborhood $\Omega_{\Sigma}$ of $\Sigma$, and that $\mathcal{T}_{\tilde{h}}(\Sigma)$ is quasi-uniform.

Lemma 4.6. There exist $C_{0}, \tilde{\beta}_{\mathrm{D}}>0$, independent of $h, h_{\Sigma}$ and $\tilde{h}$, such that for all $h_{\Sigma} \leq C_{0} \tilde{h}$, there holds

$$
S_{h}^{\mathrm{D}}\left(q_{h}^{\mathrm{D}}, \xi_{\tilde{h}}\right):=\sup _{\substack{\boldsymbol{v}_{h}^{\mathrm{D}} \in \mathbf{H}_{h}^{\mathrm{D}} \\ \boldsymbol{v}_{h}^{\mathrm{D}} \neq \mathbf{0}}} \frac{\int_{\Omega_{\mathrm{D}}} q_{h}^{\mathrm{D}} \operatorname{div} \boldsymbol{v}_{h}^{\mathrm{D}}+\left\langle\boldsymbol{v}_{h}^{\mathrm{D}} \cdot \boldsymbol{n}, \xi_{\tilde{h}}\right\rangle_{\Sigma}}{\left\|\boldsymbol{v}_{h}^{\mathrm{D}}\right\|_{\operatorname{div} ; \Omega_{\mathrm{D}}}} \geq \widetilde{\beta}_{\mathrm{D}}\left\{\left\|q_{h}^{\mathrm{D}}\right\|_{0, \Omega_{\mathrm{D}}}+\left\|\xi_{\tilde{h}}\right\|_{1 / 2, \Sigma}\right\}
$$

for all $\left(q_{h}^{\mathrm{D}}, \xi_{\tilde{h}}\right) \in \mathrm{Q}_{h, 0}^{\mathrm{D}} \times \mathrm{Q}_{\tilde{h}}^{\Sigma}$. 
Proof. We begin by observing that

$$
S_{h}^{\mathrm{D}}\left(q_{h}^{\mathrm{D}}, \xi_{\tilde{h}}\right) \geq \sup _{\substack{\boldsymbol{v}_{h}^{\mathrm{D}} \in \mathbf{H}_{h}^{\mathrm{D}} \\ \boldsymbol{v}_{h}^{\mathrm{D}} \neq \mathbf{0}}} \frac{\int_{\Omega_{\mathrm{D}}} q_{h}^{\mathrm{D}} \operatorname{div} \boldsymbol{v}_{h}^{\mathrm{D}}}{\left\|\boldsymbol{v}_{h}^{\mathrm{D}}\right\|_{\operatorname{div} ; \Omega_{\mathrm{D}}}}-\left\|\xi_{\tilde{h}}\right\|_{1 / 2, \Sigma}
$$

Then according to the results in [13, Chapter IV] (see also [20, Section 4.2]), we know that there exists $C_{\mathrm{D}}>0$, independent of $h, h_{\Sigma}$ and $\tilde{h}$, such that

$$
\sup _{\substack{\boldsymbol{v}_{h}^{\mathrm{D}} \in \mathbf{H}_{h}^{\mathrm{D}} \\ \boldsymbol{v}_{h}^{\mathrm{D}} \neq \mathbf{0}}} \frac{\int_{\Omega_{\mathrm{D}}} q_{h}^{\mathrm{D}} \operatorname{div} \boldsymbol{v}_{h}^{\mathrm{D}}}{\left\|\boldsymbol{v}_{h}^{\mathrm{D}}\right\|_{\operatorname{div} ; \Omega_{\mathrm{D}}}} \geq C_{\mathrm{D}}\left\|q_{h}^{\mathrm{D}}\right\|_{0, \Omega_{\mathrm{D}}} \quad \forall q_{h}^{\mathrm{D}} \in \mathrm{Q}_{0, h}^{\mathrm{D}},
$$

and hence

$$
S_{h}^{\mathrm{D}}\left(q_{h}^{\mathrm{D}}, \xi_{\tilde{h}}\right) \geq C_{\mathrm{D}}\left\|q_{h}^{\mathrm{D}}\right\|_{0, \Omega_{\mathrm{D}}}-\left\|\xi_{\tilde{h}}\right\|_{1 / 2, \Sigma} \quad \forall\left(q_{h}^{\mathrm{D}}, \xi_{\tilde{h}}\right) \in \mathrm{Q}_{h, 0}^{\mathrm{D}} \times \mathrm{Q}_{\tilde{h}}^{\Sigma} .
$$

On the other hand, we know from Lemma 4.4, that there exist a linear operator $\mathcal{L}_{h}: \Phi_{h}(\Sigma) \rightarrow \mathbf{H}_{h}^{\mathrm{D}}$ and a constant $C_{\mathcal{L}}>0$, independent of $h$, such that for each $\psi_{h} \in \Phi_{h}(\Sigma)$ there hold

$$
\mathcal{L}_{h}\left(\psi_{h}\right) \cdot \boldsymbol{n}=\psi_{h} \quad \text { on } \quad \Sigma, \quad\left\|\mathcal{L}_{h}\left(\psi_{h}\right)\right\|_{\operatorname{div}, \Omega_{\mathrm{D}}} \leq C_{\mathcal{L}}\left\|\psi_{h}\right\|_{-1 / 2, \Sigma}, \quad \text { and } \quad \operatorname{div} \mathcal{L}_{h}\left(\psi_{h}\right) \in P_{0}\left(\Omega_{\mathrm{D}}\right) .
$$

In this way, we deduce that

$$
S_{h}^{\mathrm{D}}\left(q_{h}^{\mathrm{D}}, \xi_{\tilde{h}}\right) \geq \frac{\int_{\Omega_{\mathrm{D}}} q_{h}^{\mathrm{D}} \operatorname{div} \mathcal{L}_{h}\left(\psi_{h}\right)+\left\langle\mathcal{L}_{h}\left(\psi_{h}\right) \cdot \boldsymbol{n}, \xi_{\tilde{h}}\right\rangle_{\Sigma}}{\left\|\mathcal{L}_{h}\left(\psi_{h}\right)\right\|_{\text {div } ; \Omega_{\mathrm{D}}}} \quad \forall \psi_{h} \in \Phi_{h}(\Sigma),
$$

from which, using that $\operatorname{div} \mathcal{L}_{h}\left(\psi_{h}\right) \in P_{0}\left(\Omega_{\mathrm{D}}\right)$ and that $q_{h}^{\mathrm{D}} \in \mathrm{Q}_{h, 0}^{\mathrm{D}}$, it follows that

$$
S_{h}^{\mathrm{D}}\left(q_{h}^{\mathrm{D}}, \xi_{\tilde{h}}\right) \geq \frac{\left|\left\langle\mathcal{L}_{h}\left(\psi_{h}\right) \cdot \boldsymbol{n}, \xi_{\tilde{h}}\right\rangle_{\Sigma}\right|}{\left\|\mathcal{L}_{h}\left(\psi_{h}\right)\right\|_{\operatorname{div}, \Omega_{\mathrm{D}}}} \geq \frac{1}{C_{\mathcal{L}}} \frac{\left|\left\langle\psi_{h}, \xi_{\tilde{h}}\right\rangle_{\Sigma}\right|}{\left\|\psi_{h}\right\|_{-1 / 2, \Sigma}} \quad \forall \psi_{h} \in \Phi_{h}(\Sigma),
$$

and hence

$$
S_{h}^{\mathrm{D}}\left(q_{h}^{\mathrm{D}}, \xi_{\tilde{h}}\right) \geq \frac{1}{C_{\mathcal{L}}} \sup _{\substack{\psi_{h} \in \Phi_{h}(\Sigma) \\ \psi_{h} \neq 0}} \frac{\left\langle\psi_{h}, \xi_{\tilde{h}}\right\rangle_{\Sigma}}{\left\|\psi_{h}\right\|_{-1 / 2, \Sigma}} \quad \forall \xi_{\tilde{h}} \in \mathrm{Q}_{\tilde{h}}^{\Sigma}
$$

Therefore, (4.33) and a straightforward application of Lemma 4.5 imply the existence of $\widetilde{C}_{\mathrm{D}}>0$, independent of $h, h_{\Sigma}$ and $\tilde{h}$, such that for all $h_{\Sigma} \leq C_{0} \tilde{h}$ there holds

$$
S_{h}^{\mathrm{D}}\left(q_{h}^{\mathrm{D}}, \xi_{\tilde{h}}\right) \geq \widetilde{C}_{\mathrm{D}}\left\|\xi_{\tilde{h}}\right\|_{1 / 2, \Sigma} \quad \forall \xi_{\tilde{h}} \in \mathrm{Q}_{\tilde{h}}^{\Sigma}
$$

Finally, it easy to see that estimates (4.32) and (4.34) imply the discrete inf-sup condition (4.31), thus finishing the proof.

Lemma 4.7. There exist $C_{0}, \tilde{\beta}_{\mathrm{B}}>0$, independent of $h, h_{\Sigma}$ and $\tilde{h}$, such that for all $h_{\Sigma} \leq C_{0} \tilde{h}$ there holds

$$
S_{h}^{\mathrm{B}}\left(q_{h}^{\mathrm{B}}, \xi_{\tilde{h}}\right):=\sup _{\substack{\boldsymbol{v}_{h}^{\mathrm{B}} \in \mathbf{H}_{h}^{\mathrm{B}} \\ \boldsymbol{v}_{h}^{\mathrm{B}} \neq \mathbf{0}}} \frac{\int_{\Omega_{\mathrm{B}}} q_{h}^{\mathrm{B}} \operatorname{div} \boldsymbol{v}_{h}^{\mathrm{B}}-\left\langle\boldsymbol{v}_{h}^{\mathrm{B}} \cdot \boldsymbol{n}, \xi_{\tilde{h}}\right\rangle_{\Sigma}}{\left\|\boldsymbol{v}_{h}^{\mathrm{B}}\right\|_{\operatorname{div} ; \Omega_{\mathrm{B}}}} \geq \widetilde{\beta}_{\mathrm{B}}\left\{\left\|q_{h}^{\mathrm{B}}\right\|_{0, \Omega_{\mathrm{B}}}+\left\|\xi_{\tilde{h}}\right\|_{1 / 2, \Sigma}\right\}
$$

for all $\left(q_{h}^{\mathrm{B}}, \xi_{\tilde{h}}\right) \in \mathrm{Q}_{h, 0}^{\mathrm{B}} \times \mathrm{Q}_{\tilde{h}}^{\Sigma}$.

Proof. It proceeds exactly as the proof of Lemma 4.6 by replacing $\Omega_{\mathrm{D}}, \Gamma_{\mathrm{D}}, \mathrm{Q}_{h, 0}^{\mathrm{D}}$ and $\mathbf{H}_{h}^{\mathrm{D}}$ by $\Omega_{\mathrm{B}}, \Gamma_{\mathrm{B}}, \mathrm{Q}_{h, 0}^{\mathrm{B}}$ and $\mathbf{H}_{h}^{\mathrm{B}}$, respectively.

The following theorem provides the rate of convergence of our Galerkin scheme (4.4). 
Theorem 4.2. Let $\mathbf{H}_{h}:=\mathbf{H}_{h}^{\mathrm{B}} \times \mathbf{H}_{0, h}^{\mathrm{B}} \times \mathbf{H}_{h}^{\mathrm{D}}$ and $\mathrm{Q}_{h, 0}:=\mathrm{Q}_{h, 0}^{\mathrm{B}} \times \mathrm{Q}_{h}^{\mathrm{D}} \times \mathrm{Q}_{\tilde{h}}^{\Sigma}$ be the subspaces specified above, and let $(\overrightarrow{\boldsymbol{u}}, \vec{p}):=\left(\left(\boldsymbol{u}_{\mathrm{B}}, \boldsymbol{\omega}_{\mathrm{B}}, \boldsymbol{u}_{\mathrm{D}}\right),\left(p_{\mathrm{B}}, p_{\mathrm{D}}, \lambda\right)\right) \in \mathbf{H} \times \mathbf{Q}_{0}$ and $\left(\overrightarrow{\boldsymbol{u}}_{h}, \vec{p}_{h}\right):=\left(\left(\boldsymbol{u}_{h}^{\mathrm{B}}, \boldsymbol{\omega}_{h}^{\mathrm{B}}, \boldsymbol{u}_{h}^{\mathrm{D}}\right),\left(p_{h}^{\mathrm{B}}, p_{h}^{\mathrm{D}}, \lambda_{\tilde{h}}\right)\right) \in \mathbf{H}_{h} \times \mathbf{Q}_{0, h}$ be the unique solutions of the continuous and discrete problems (3.10) and (4.4), respectively. Assume that $\boldsymbol{u}_{\star} \in \mathbf{H}^{\delta}\left(\Omega_{\star}\right), \operatorname{div} \boldsymbol{u}_{\star} \in \mathrm{H}^{\delta}\left(\Omega_{\star}\right), p_{\star} \in \mathrm{H}^{\delta}\left(\Omega_{\star}\right)$ where $\star \in\{\mathrm{B}, \mathrm{D}\}, \boldsymbol{\omega}_{\mathrm{B}} \in \mathbf{H}^{\delta}\left(\mathbf{c u r l} ; \Omega_{\mathrm{B}}\right)$ and $\lambda \in \mathrm{H}^{1 / 2+\delta}(\Sigma)$, for some $\delta \in(1 / 2,1]$. Then, there exists $C>0$ and $\widetilde{C}>0$ independent of $h$ and $\tilde{h}$ such that

$$
\begin{gathered}
\left\|(\overrightarrow{\boldsymbol{u}}, \vec{p})-\left(\overrightarrow{\boldsymbol{u}}_{h}, \vec{p}_{h}\right)\right\|_{\mathbf{H}} \leq C h^{\delta}\left\{\left\|\boldsymbol{u}_{\mathrm{B}}\right\|_{\delta, \Omega_{\mathrm{B}}}+\left\|\operatorname{div}\left(\boldsymbol{u}_{\mathrm{B}}\right)\right\|_{\delta, \Omega_{\mathrm{B}}}+\left\|\boldsymbol{\omega}_{\mathrm{B}}\right\|_{\mathbf{H}^{\delta}\left(\mathbf{c u r l} ; \Omega_{\mathrm{B}}\right)}+\left\|\boldsymbol{u}_{\mathrm{D}}\right\|_{\delta, \Omega_{\mathrm{D}}}\right. \\
\left.+\left\|\operatorname{div}\left(\boldsymbol{u}_{\mathrm{D}}\right)\right\|_{\delta, \Omega_{\mathrm{D}}}+\left\|p_{\mathrm{B}}\right\|_{\delta, \Omega_{\mathrm{B}}}+\left\|p_{\mathrm{D}}\right\|_{\delta, \Omega_{\mathrm{D}}}\right\}+\widetilde{C} \tilde{h}^{\delta}\|\lambda\|_{\delta+1 / 2, \Sigma}
\end{gathered}
$$

Proof. It follows from the Céa estimate (4.15) and the approximation properties $\left(\mathrm{AP}_{h}^{\boldsymbol{u}_{\star}}\right),\left(\mathrm{AP}_{h}^{p_{\star}}\right),\left(\mathrm{AP}_{h}^{\boldsymbol{\omega}_{\mathrm{B}}}\right)$ and $\left(\mathrm{AP}_{h}^{\lambda}\right)$.

We end this section by remarking that the analysis from Section 4.2 can be extended without difficulties, to Raviart-Thomas and Nédélec spaces of higher order.

\section{An augmented mixed formulation}

In this section we propose an augmented variational formulation of problem (3.10). Indeed, though many finite element subspaces $\mathbf{H}_{0, h}^{\mathrm{B}} \subseteq \mathbf{H}_{0}\left(\mathbf{c u r l} ; \Omega_{\mathrm{B}}\right)$ and $\mathbf{H}_{h}^{\mathrm{B}} \subseteq \mathbf{H}_{\mathrm{B}}\left(\right.$ div; $\left.\Omega_{\mathrm{B}}\right)$ do satisfy (H.3), we would like to explore the possibility of getting rid of that assumption. To this end, we suggest to enrich the mixed variational formulation (3.10) with a residual arising from the Brinkman momentum equation in (2.1). More precisely, we include into the variational problem (3.10) the following Galerkin least-squares equation in $\Omega_{\mathrm{B}}$ :

$$
\kappa \int_{\Omega_{\mathrm{B}}}\left(\alpha \boldsymbol{u}_{\mathrm{B}}+\nu \operatorname{curl} \boldsymbol{\omega}_{\mathrm{B}}+\nabla p_{\mathrm{B}}-\boldsymbol{f}_{\mathrm{B}}\right) \cdot \operatorname{curl} \boldsymbol{z}_{\mathrm{B}}=0 \quad \forall \boldsymbol{z}_{\mathrm{B}} \in \mathbf{H}_{0}\left(\operatorname{curl} ; \Omega_{\mathrm{B}}\right)
$$

where $\kappa$ is a positive parameter to be specified later. Actually, integrating by parts, and using again that $\operatorname{curl} \boldsymbol{z}_{\mathrm{B}} \in \mathbf{H}_{0}\left(\operatorname{div} ; \Omega_{\mathrm{B}}\right)$ for each $\boldsymbol{z}_{\mathrm{B}} \in \mathbf{H}_{0}\left(\mathbf{c u r l} ; \Omega_{\mathrm{B}}\right)$ (cf. [27, Chapter I, Section 2.3, Remark 2.5]), we easily find that

$$
\int_{\Omega_{\mathrm{B}}} \nabla p_{\mathrm{B}} \cdot \operatorname{curl} \boldsymbol{z}_{\mathrm{B}}=0 \quad \forall \boldsymbol{z}_{\mathrm{B}} \in \mathbf{H}_{0}\left(\operatorname{curl} ; \Omega_{\mathrm{B}}\right),
$$

whence (5.1) can be recast in the form

$$
\kappa \alpha \int_{\Omega_{\mathrm{B}}} \boldsymbol{u}_{\mathrm{B}} \cdot \operatorname{curl} z_{\mathrm{B}}+\kappa \nu \int_{\Omega_{\mathrm{B}}} \operatorname{curl} \boldsymbol{\omega}_{\mathrm{B}} \cdot \operatorname{curl} z_{\mathrm{B}}=\kappa \int_{\Omega_{\mathrm{B}}} \boldsymbol{f}_{\mathrm{B}} \cdot \operatorname{curl} z_{\mathrm{B}} \quad \forall z_{\mathrm{B}} \in \mathbf{H}_{0}\left(\operatorname{curl} ; \Omega_{\mathrm{B}}\right) .
$$

In this way, adding (5.2) to the first equation of (3.10), we obtain the following augmented variational formulation: Find $\overrightarrow{\boldsymbol{u}}:=\left(\boldsymbol{u}_{\mathrm{B}}, \boldsymbol{\omega}_{\mathrm{B}}, \boldsymbol{u}_{\mathrm{D}}\right) \in \mathbf{H}$ and $\vec{p}:=\left(p_{\mathrm{B}}, p_{\mathrm{D}}, \lambda\right) \in \mathbf{Q}_{0}$ such that

$$
\begin{array}{lll}
\mathcal{A}(\overrightarrow{\boldsymbol{u}}, \overrightarrow{\boldsymbol{v}})+\mathcal{B}(\overrightarrow{\boldsymbol{v}}, \vec{p}) & =\mathcal{F}(\overrightarrow{\boldsymbol{v}}) & \forall \overrightarrow{\boldsymbol{v}}:=\left(\boldsymbol{v}_{\mathrm{B}}, \boldsymbol{z}_{\mathrm{B}}, \boldsymbol{v}_{\mathrm{D}}\right) \in \mathbf{H}, \\
\mathcal{B}(\overrightarrow{\boldsymbol{u}}, \vec{q}) & =\mathcal{G}(\vec{q}) & \forall \vec{q}:=\left(q_{\mathrm{B}}, q_{\mathrm{D}}, \xi\right) \in \mathbf{Q}_{0},
\end{array}
$$

where

$$
\begin{gathered}
\mathcal{A}(\overrightarrow{\boldsymbol{u}}, \overrightarrow{\boldsymbol{v}}):=\alpha \int_{\Omega_{\mathrm{B}}} \boldsymbol{u}_{\mathrm{B}} \cdot \boldsymbol{v}_{\mathrm{B}}+\nu \int_{\Omega_{\mathrm{B}}} \boldsymbol{\omega}_{\mathrm{B}} \cdot \boldsymbol{z}_{\mathrm{B}}+\kappa \nu \int_{\Omega_{\mathrm{B}}} \operatorname{curl} \boldsymbol{\omega}_{\mathrm{B}} \cdot \operatorname{curl} \boldsymbol{z}_{\mathrm{B}} \\
+\nu \int_{\Omega_{\mathrm{B}}} \boldsymbol{v}_{\mathrm{B}} \cdot \operatorname{curl} \boldsymbol{\omega}_{\mathrm{B}}+(\kappa \alpha-\nu) \int_{\Omega_{\mathrm{B}}} \boldsymbol{u}_{\mathrm{B}} \cdot \operatorname{curl} \boldsymbol{z}_{\mathrm{B}}+\mu \int_{\Omega_{\mathrm{D}}} \boldsymbol{u}_{\mathrm{D}} \cdot \boldsymbol{v}_{\mathrm{D}} \quad \forall(\overrightarrow{\boldsymbol{u}}, \overrightarrow{\boldsymbol{v}}) \in \mathbf{H} \times \mathbf{H}, \\
\mathcal{F}(\overrightarrow{\boldsymbol{v}}):=\int_{\Omega_{\mathrm{B}}} \boldsymbol{f}_{\mathrm{B}} \cdot \boldsymbol{v}_{\mathrm{B}}+\int_{\Omega_{\mathrm{D}}} \boldsymbol{f}_{\mathrm{D}} \cdot \boldsymbol{v}_{\mathrm{D}}+\kappa \int_{\Omega_{\mathrm{B}}} \boldsymbol{f}_{\mathrm{B}} \cdot \operatorname{curl} \boldsymbol{z}_{\mathrm{B}} \quad \forall \overrightarrow{\boldsymbol{v}} \in \mathbf{H},
\end{gathered}
$$

$\mathcal{B}=\mathbf{b}$, and $\mathcal{G}=\mathbf{G}=\mathbf{0}$.

In what follows we address the solvability of (5.3). We first observe that the continuous inf-sup condition for $\mathcal{B}$ on $\mathbf{H} \times \mathbf{Q}_{0}$ is already proved by Lemma 3.4. In turn, the continuous kernel of $\mathcal{B}$ is certainly given by $\mathbf{V}$ (cf. (3.13) - (3.15)). Then, we have the following result establishing the ellipticity of $\mathcal{A}$ on $\mathbf{V}_{\mathrm{B}, \mathrm{D}}$ and hence on $\mathbf{V}$. 
Lemma 5.1. Assume that the stabilization parameter $\kappa \in(0,2 \delta)$ with $\delta \in\left(0, \frac{2 \nu}{\alpha}\right)$. Then, there exists $\varrho>0$, depending on $\kappa$ and $\delta$, such that

$$
\mathcal{A}(\overrightarrow{\boldsymbol{v}}, \overrightarrow{\boldsymbol{v}}) \geq \varrho\|\overrightarrow{\boldsymbol{v}}\|_{\mathbf{H}}^{2} \quad \forall \overrightarrow{\boldsymbol{v}} \in \mathbf{V}_{\mathrm{B}, \mathrm{D}} .
$$

Proof. Given $\overrightarrow{\boldsymbol{v}}:=\left(\boldsymbol{v}_{\mathrm{B}}, \boldsymbol{z}_{\mathrm{B}}, \boldsymbol{v}_{\mathrm{D}}\right) \in \mathbf{V}_{\mathrm{B}, \mathrm{D}}$, we obtain from the definition of $\mathcal{A}$ (cf. (5.4)) and the CauchySchwarz inequality, that

$$
\begin{gathered}
\mathcal{A}(\overrightarrow{\boldsymbol{v}}, \overrightarrow{\boldsymbol{v}})=\alpha\left\|\boldsymbol{v}_{\mathrm{B}}\right\|_{0, \Omega_{\mathrm{B}}}^{2}+\nu\left\|\boldsymbol{z}_{\mathrm{B}}\right\|_{0, \Omega_{\mathrm{B}}}^{2}+\kappa \nu\left\|\operatorname{curl} \boldsymbol{z}_{\mathrm{B}}\right\|_{0, \Omega_{\mathrm{B}}}^{2}+\kappa \alpha \int_{\Omega_{\mathrm{B}}} \boldsymbol{v}_{\mathrm{B}} \cdot \operatorname{curl} \boldsymbol{z}_{\mathrm{B}}+\mu\left\|\boldsymbol{v}_{\mathrm{D}}\right\|_{0, \Omega_{\mathrm{D}}}^{2} \\
\geq \alpha\left\|\boldsymbol{v}_{\mathrm{B}}\right\|_{0, \Omega_{\mathrm{B}}}^{2}+\nu\left\|\boldsymbol{z}_{\mathrm{B}}\right\|_{0, \Omega_{\mathrm{B}}}^{2}+\kappa \nu\left\|\operatorname{curl} \boldsymbol{z}_{\mathrm{B}}\right\|_{0, \Omega_{\mathrm{B}}}^{2}-\kappa \alpha\left\|\boldsymbol{v}_{\mathrm{B}}\right\|_{0, \Omega_{\mathrm{B}}}\left\|\operatorname{curl} \boldsymbol{z}_{\mathrm{B}}\right\|_{0, \Omega_{\mathrm{B}}}+\mu\left\|\boldsymbol{v}_{\mathrm{D}}\right\|_{0, \Omega_{\mathrm{D}}}^{2} .
\end{gathered}
$$

Next, for each $\delta>0$ we find that

$$
-\kappa \alpha\left\|\boldsymbol{v}_{\mathrm{B}}\right\|_{0, \Omega_{\mathrm{B}}}\left\|\operatorname{curl} \boldsymbol{z}_{\mathrm{B}}\right\|_{0, \Omega_{\mathrm{B}}} \geq-\frac{\kappa \alpha}{2 \delta}\left\|\boldsymbol{v}_{\mathrm{B}}\right\|_{0, \Omega_{\mathrm{B}}}^{2}-\frac{\delta \kappa \alpha}{2}\left\|\operatorname{curl} \boldsymbol{z}_{\mathrm{B}}\right\|_{0, \Omega_{\mathrm{B}}}^{2},
$$

which, replaced back into the foregoing estimate, yields

$$
\mathcal{A}(\overrightarrow{\boldsymbol{v}}, \overrightarrow{\boldsymbol{v}}) \geq \alpha\left(1-\frac{\kappa}{2 \delta}\right)\left\|\boldsymbol{v}_{\mathrm{B}}\right\|_{0, \Omega_{\mathrm{B}}}^{2}+\nu\left\|\boldsymbol{z}_{\mathrm{B}}\right\|_{0, \Omega_{\mathrm{B}}}^{2}+\kappa\left(\nu-\frac{\delta \alpha}{2}\right)\left\|\operatorname{curl} \boldsymbol{z}_{\mathrm{B}}\right\|_{0, \Omega_{\mathrm{B}}}^{2}+\mu\left\|\boldsymbol{v}_{\mathrm{D}}\right\|_{0, \Omega_{\mathrm{D}}}^{2} .
$$

Next, using (3.18) and noting that $\left\|\boldsymbol{v}_{\mathrm{D}}\right\|_{0, \Omega_{\mathrm{B}}}^{2}=\left\|\boldsymbol{v}_{\mathrm{D}}\right\|_{\text {div } ; \Omega_{\mathrm{D}}}^{2}$, we obtain

$$
\mathcal{A}(\overrightarrow{\boldsymbol{v}}, \overrightarrow{\boldsymbol{v}}) \geq \alpha\left(1-\frac{\kappa}{2 \delta}\right) \varrho_{0}^{2}\left\|\boldsymbol{v}_{\mathrm{B}}\right\|_{\mathrm{div}, \Omega_{\mathrm{B}}}^{2}+\nu\left\|\boldsymbol{z}_{\mathrm{B}}\right\|_{0, \Omega_{\mathrm{B}}}^{2}+\kappa\left(\nu-\frac{\delta \alpha}{2}\right)\left\|\operatorname{curl} \boldsymbol{z}_{\mathrm{B}}\right\|_{0, \Omega_{\mathrm{B}}}^{2}+\mu\left\|\boldsymbol{v}_{\mathrm{D}}\right\|_{\mathrm{div}, \Omega_{\mathrm{D}}}^{2} .
$$

Hence, since $1-\frac{\kappa}{2 \delta}>0$ and $\nu-\frac{\delta \alpha}{2}>0$, we conclude that

$$
\mathcal{A}(\overrightarrow{\boldsymbol{v}}, \overrightarrow{\boldsymbol{v}}) \geq \varrho\|\overrightarrow{\boldsymbol{v}}\|_{\mathbf{H}}^{2} \quad \overrightarrow{\boldsymbol{v}} \in \mathbf{V}_{\mathrm{B}, \mathrm{D}}
$$

where $\varrho:=\min \left\{\alpha\left(1-\frac{\kappa}{2 \delta}\right) \varrho_{0}^{2}, \nu, \kappa\left(\nu-\frac{\delta \alpha}{2}\right), \mu\right\}$.

Note that, taking in particular $\kappa=\delta=\frac{\nu}{\alpha}$, we obtain the optimal ellipticity constant

$$
\varrho:=\frac{1}{2} \min \left\{\alpha \varrho_{0}^{2}, 2 \nu, \kappa \nu, 2 \mu\right\} .
$$

The foregoing analysis yields the following main result.

Theorem 5.1. Assume that $\mathbf{f}_{\mathrm{D}} \in \mathbf{L}^{2}\left(\Omega_{\mathrm{D}}\right), \mathbf{f}_{\mathrm{B}} \in \mathbf{L}^{2}\left(\Omega_{\mathrm{B}}\right)$, and that $\kappa$ satisfies the assumption from Lemma 5.1. Then there exists a unique $(\overrightarrow{\boldsymbol{u}}, \vec{p}):=\left(\left(\boldsymbol{u}_{\mathrm{B}}, \boldsymbol{\omega}_{\mathrm{B}}, \boldsymbol{u}_{\mathrm{D}}\right),\left(p_{\mathrm{B}}, p_{\mathrm{D}}, \lambda\right)\right) \in \mathbf{H} \times \mathbf{Q}_{0}$ solution of the augmented mixed formulation (5.3). Moreover, there exists $C>0$ such that

$$
\|\overrightarrow{\boldsymbol{u}}\|_{\mathbf{H}}+\|\vec{p}\|_{\mathbf{Q}} \leq C\left\{\left\|\boldsymbol{f}_{\mathrm{D}}\right\|_{0, \Omega_{\mathrm{D}}}+\left\|\boldsymbol{f}_{\mathrm{B}}\right\|_{0, \Omega_{\mathrm{B}}}\right\} .
$$

Proof. Thanks to Lemmata 3.4 and 5.1, the proof is a straightforward application of the continuous BabuškaBrezzi theory.

We now look at the Galerkin scheme of (5.3). More precisely, employing the same generic finite elements subspaces and related notations introduced in Section 4.1, we now consider the augmented mixed finite element scheme: Find $\overrightarrow{\boldsymbol{u}}_{h}:=\left(\boldsymbol{u}_{h}^{\mathrm{B}}, \boldsymbol{\omega}_{h}^{\mathrm{B}}, \boldsymbol{u}_{h}^{\mathrm{D}}\right) \in \mathbf{H}_{h}$ and $\vec{p}_{h}:=\left(p_{h}^{\mathrm{B}}, p_{h}^{\mathrm{D}}, \lambda_{h}\right) \in \mathbf{Q}_{0, h}$ such that

$$
\begin{array}{lll}
\mathcal{A}\left(\overrightarrow{\boldsymbol{u}}_{h}, \overrightarrow{\boldsymbol{v}}_{h}\right)+\mathcal{B}\left(\overrightarrow{\boldsymbol{v}}_{h}, \vec{p}_{h}\right) & =\mathcal{F}\left(\overrightarrow{\boldsymbol{v}}_{h}\right) & \forall \overrightarrow{\boldsymbol{v}}_{h}:=\left(\boldsymbol{v}_{h}^{\mathrm{B}}, \boldsymbol{z}_{h}^{\mathrm{B}}, \boldsymbol{v}_{h}^{\mathrm{D}}\right) \in \mathbf{H}_{h}, \\
\mathcal{B}\left(\overrightarrow{\boldsymbol{u}}_{h}, \vec{q}_{h}\right) & =\mathcal{G}\left(\vec{q}_{h}\right) & \forall \vec{q}_{h}:=\left(q_{h}^{\mathrm{B}}, q_{h}^{\mathrm{D}}, \xi_{h}\right) \in \mathbf{Q}_{0, h} .
\end{array}
$$


Then, assuming that hypotheses (H.0), (H.1), and (H.2) from Section 4 are satisfied, we certainly deduce that $\mathcal{B}$ verifies the discrete inf-sup condition on $\mathbf{H}_{h} \times \mathbf{Q}_{0, h}$ (cf. Lemma 4.1), the discrete kernel of $\mathcal{B}$ is given again by $\mathbf{V}_{h}=\mathbf{V}_{\mathrm{B}, \mathrm{D}}^{h} \cap \mathbf{V}_{\Sigma}^{h}$ (cf. (4.9) - (4.11)), and hence, since $\mathbf{V}_{\mathrm{B}, \mathrm{D}}^{h}$ is contained in $\mathbf{V}_{\mathrm{B}, \mathrm{D}}$, the bilinear form $\mathcal{A}$ is elliptic in $\mathbf{V}_{\text {B.D }}^{h}$ (cf. Lemma 5.1) and therefore in $\mathbf{V}_{h}$. Consequently, a straightforward application of the discrete Babuška-Brezzi theory allows to conclude the following result.

Theorem 5.2. Assume that $\mathbf{f}_{\mathrm{D}} \in \mathbf{L}^{2}\left(\Omega_{\mathrm{D}}\right)$ and $\mathbf{f}_{\mathrm{B}} \in \mathbf{L}^{2}\left(\Omega_{\mathrm{B}}\right)$. In addition, suppose that (H.0), (H.1), and (H.2) hold. Then there exists a unique $\left(\overrightarrow{\boldsymbol{u}}_{h}, \vec{p}_{h}\right):=\left(\left(\boldsymbol{u}_{h}^{\mathrm{B}}, \boldsymbol{\omega}_{h}^{\mathrm{B}}, \boldsymbol{u}_{h}^{\mathrm{D}}\right),\left(p_{h}^{\mathrm{B}}, p_{h}^{\mathrm{D}}, \lambda_{h}\right)\right) \in \mathbf{H}_{h} \times \mathbf{Q}_{0, h}$ solution of the augmented Galerkin scheme (5.9). Moreover, there exist $C_{1}, C_{2}>0$, independent of $h$, such that

$$
\left\|\overrightarrow{\boldsymbol{u}}_{h}\right\|_{\mathbf{H}}+\left\|\vec{p}_{h}\right\|_{\mathbf{Q}} \leq C_{1}\left\{\left\|\boldsymbol{f}_{\mathrm{D}}\right\|_{0, \Omega_{\mathrm{D}}}+\left\|\boldsymbol{f}_{\mathrm{B}}\right\|_{0, \Omega_{\mathrm{B}}}\right\},
$$

and

$$
\left\|(\overrightarrow{\boldsymbol{u}}, \vec{p})-\left(\overrightarrow{\boldsymbol{u}}_{h}, \vec{p}_{h}\right)\right\|_{\mathbf{H} \times \mathbf{Q}} \leq C_{2}\left\{\operatorname{dist}\left(\overrightarrow{\boldsymbol{u}}, \mathbf{H}_{h}\right)+\operatorname{dist}\left(\vec{p}, \mathbf{Q}_{0, h}\right)\right\} .
$$

We end this section by providing a specific example of finite element subspaces satisfying (H.0), (H.1), and (H.2), but not (H.3), whence only the augmented formulation described in this section can be employed with them. In fact, for each $K \in \mathcal{T}_{h}\left(\Omega_{\mathrm{B}}\right)$ we now let $\mathbb{N D}_{2}(K)$ be the local Nédélec space of order 2 , that is

$$
\mathbb{N D}_{2}(K):=\mathbf{P}_{1}(K) \oplus \widetilde{\mathbf{P}}_{1}(K) \times \boldsymbol{x},
$$

where $\widetilde{\mathbf{P}}_{1}(K)$ is the space of polynomials of degree $=1$, and introduce

$$
\mathbf{H}_{0, h}^{\mathrm{B}}:=\left\{\boldsymbol{z}_{h}^{\mathrm{B}} \in \mathbf{H}_{0}\left(\operatorname{curl} ; \Omega_{\mathrm{B}}\right):\left.\quad \boldsymbol{z}_{h}^{\mathrm{B}}\right|_{K} \in \mathbb{N D}_{2}(K) \quad \forall K \in \mathcal{T}_{h}\left(\Omega_{\mathrm{B}}\right)\right\} .
$$

In turn, the remaining subspaces $\mathbf{H}_{h}^{\mathrm{B}}, \mathbf{H}_{h}^{\mathrm{D}}, \mathrm{Q}_{h, 0}^{\mathrm{B}}, \mathrm{Q}_{h}^{\mathrm{D}}$, and $\mathrm{Q}_{h}^{\Sigma}$ are exactly as those defined in Section 4.2.1, that is with local Raviart-Thomas spaces of order 0 and discontinuous piecewise constant polynomials on the domains, and with continuous piecewise polynomials of degree $\leq 1$ on the interface $\Sigma$. Then, it is not difficult to see that there exist $\boldsymbol{z}_{h}^{\mathrm{B}} \in \mathbf{H}_{0, h}^{\mathrm{B}}$ for which $\operatorname{curl} \boldsymbol{z}_{h}^{\mathrm{B}}$ does not belong to $\mathbf{H}_{h}^{\mathrm{B}}$, thus confirming that (H.3) does not hold in this case. Numerical results illustrating optimal convergence rates of the augmented formulation with the aforementioned finite element subspaces are reported below in Section 6 .

\section{Numerical results}

In this section we provide three computer experiments confirming the convergence rates anticipated by Theorem 4.2 and illustrating the applicability of the method in surface-subsurface flow problems.

\subsection{Accuracy of the mixed and augmented formulations on two embedded cubes}

We start by evaluating the convergence of the fully-mixed and the augmented finite element methods applied to (2.1)-(2.2) and defined on the two cubes $\Omega_{\mathrm{B}}=\left[-r_{B}, r_{B}\right]^{3}$ and $\Omega_{\mathrm{D}}=\left[-r_{D}, r_{D}\right]^{3}$, with $r_{D}=\frac{1}{2}, r_{B}=$ $\frac{3}{20}$. Notice that this particular domain configuration does not fall exactly in the theoretical framework analyzed in this paper. However, both the continuous and discrete study could be carried out using the analogous tools as those used here. We employ the model parameters $\alpha=\mu=1, \nu=0.01$, yielding the stabilization constant $\kappa=2 \nu / \alpha=0.02$ suggested by Lemma 5.1. The convergence of the method is assessed by computing errors between the following manufactured smooth exact solutions

$$
\begin{gathered}
\boldsymbol{\omega}_{\mathrm{B}}\left(x_{1}, x_{2}, x_{3}\right)=\left(\begin{array}{c}
-3 \pi \sin \left(\pi x_{1}\right) \cos \left(\pi x_{2}\right) \cos \left(\pi x_{3}\right) \\
3 \pi \cos \left(\pi x_{1}\right) \sin \left(\pi x_{2}\right) \cos \left(\pi x_{3}\right) \\
0
\end{array}\right), \boldsymbol{u}\left(x_{1}, x_{2}, x_{3}\right)=\left(\begin{array}{c}
\cos \left(\pi x_{1}\right) \sin \left(\pi x_{2}\right) \sin \left(\pi x_{3}\right) \\
\sin \left(\pi x_{1}\right) \cos \left(\pi x_{2}\right) \sin \left(\pi x_{3}\right) \\
-2 \sin \left(\pi x_{1}\right) \sin \left(\pi x_{2}\right) \cos \left(\pi x_{3}\right)
\end{array}\right), \\
p\left(x_{1}, x_{2}, x_{3}\right)=\sin \left(\pi x_{1}\right) \sin \left(\pi x_{2}\right) \sin \left(\pi x_{3}\right), \boldsymbol{u}_{\mathrm{B}}=\left.\boldsymbol{u}\right|_{\Omega_{\mathrm{B}}}, \boldsymbol{u}_{\mathrm{D}}=\left.\boldsymbol{u}\right|_{\Omega_{\mathrm{D}}}, p_{\mathrm{B}}=\left.p\right|_{\Omega_{\mathrm{B}}}, p_{\mathrm{D}}=\left.p\right|_{\Omega_{\mathrm{D}}}, \lambda=\left.p\right|_{\Sigma},
\end{gathered}
$$

and their finite element approximations using a $\mathbb{R T}_{0}-\mathbb{N D}_{1}-\mathbb{R T}_{0}-\mathbf{P}_{0}-\mathbf{P}_{0}-\mathbf{P}_{1}$ family (and using the fully-mixed and augmented formulations), and also an augmented method based on the $\mathbb{R T}_{0}-\mathbb{N D}_{2}-\mathbb{R T}_{0}-$ 

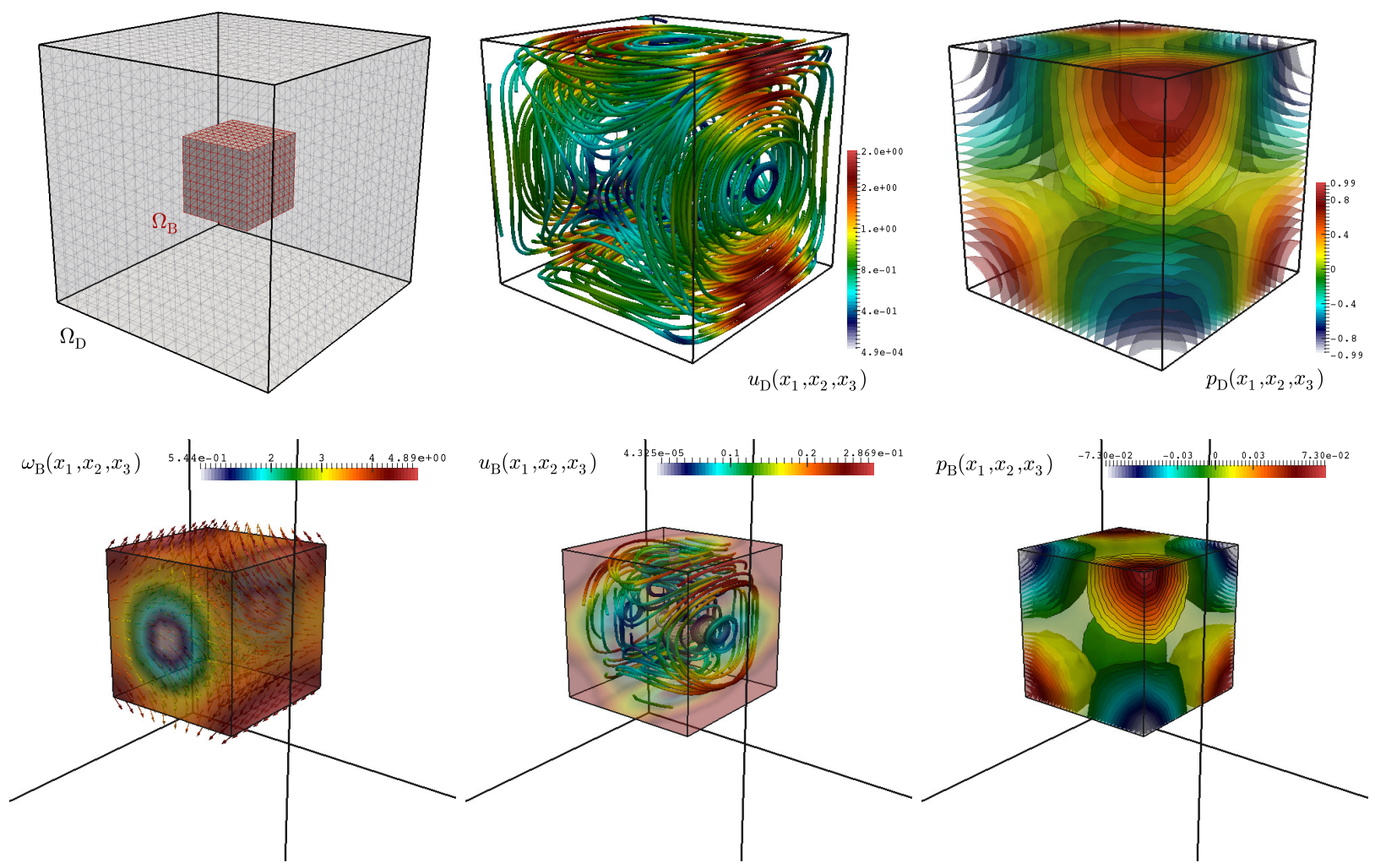

Figure 6.1: Example 1: Two-domain geometry and mesh (top left), approximated Darcy velocity streamlines (top middle), approximated Darcy pressure isosurfaces (top right), zoom of approximated Brinkman vorticity vectors (bottom left), zoom of approximated Brinkman velocity streamlines (bottom middle), and isosurfaces of the computed Brinkman pressure (bottom right).

$\mathbf{P}_{0}-\mathbf{P}_{0}-\mathbf{P}_{1}$ family, which, in particular, does not satisfy assumption (H.3). The computations are carried out on a sequence of successively refined tetrahedral meshes $\mathcal{T}_{h_{B i}}$ and $\mathcal{T}_{h_{D i}}$ of sizes $h_{B i}=r_{B} 2^{1-i}$ and $h_{D i}=r_{D} 2^{-i}$, respectively, $i=0,1, \ldots$. We adequately choose forcing terms $\boldsymbol{f}_{\mathrm{B}}=\alpha \boldsymbol{u}_{\mathrm{B}}+\mathbf{c u r l} \boldsymbol{\omega}_{\mathrm{B}}+\nabla p_{\mathrm{B}}$, $\boldsymbol{f}_{\mathrm{D}}=\mu \boldsymbol{u}_{\mathrm{D}}+\nabla p_{\mathrm{D}}$, and suitable nonhomogeneous slip velocity on $\partial \Omega$ and nonhomogeneous Dirichlet data for the tangential vorticity on $\partial \Omega_{\mathrm{B}}$, such that (2.1)-(2.2) holds. For sake of convenience we define a conforming partition for $\Sigma$, that is $\mathcal{T}_{\tilde{h}}=\mathcal{T}_{h}$. The approximate solutions are depicted in Figure 6.1 and the error history, written in terms of the quantities

$$
\begin{aligned}
& e\left(\boldsymbol{u}_{\mathrm{B}}\right):=\left\|\boldsymbol{u}_{\mathrm{B}}-\boldsymbol{u}_{\mathrm{B} h}\right\|_{\text {div }, \Omega_{\mathrm{B}}}, e\left(\boldsymbol{\omega}_{\mathrm{B}}\right):=\left\|\boldsymbol{\omega}_{\mathrm{B}}-\boldsymbol{\omega}_{\mathrm{B} h}\right\|_{\mathbf{c u r l}, \Omega_{\mathrm{B}}}, e\left(\boldsymbol{u}_{\mathrm{D}}\right):=\left\|\boldsymbol{u}_{\mathrm{D}}-\boldsymbol{u}_{\mathrm{D} h}\right\|_{\mathrm{div}, \Omega_{\mathrm{D}}}, \\
& e\left(p_{\mathrm{B}}\right):=\left\|p_{\mathrm{B}}-p_{\mathrm{B} h}\right\|_{0, \Omega_{\mathrm{B}}}, e\left(p_{\mathrm{D}}\right):=\left\|p_{\mathrm{D}}-p_{\mathrm{D}_{h}}\right\|_{0, \Omega_{\mathrm{D}}}, e(\lambda):=\left\|\lambda-\lambda_{h}\right\|_{1 / 2, \Sigma}, r(\cdot):=\frac{\log (e(\cdot) / \hat{e}(\cdot))}{\log (h / \hat{h})},
\end{aligned}
$$

is reported in Table 6.1, where $e, \hat{e}$ denote errors on two consecutive meshes of sizes $h=\max \left\{h_{B}, h_{D}\right\}$ and $\hat{h}$. We observe that all studied methods deliver optimal convergence rates for vorticity, velocity and pressure in the corresponding norms.

\subsection{Flow into a cracked porous medium}

Our second example focuses on the simulation of flow in a porous medium with a smoothed V-shaped crack, similar to the 2D simulations presented in the Stokes-Darcy examples of [9, Section 7.1] and [14, Section 6.3]. The full domain is the box $\Omega=[0,2] \times[0,0.2] \times[0,1]$, the Brinkman domain on the top is $0.75 \leq x_{1} \leq 1.25$ and goes down to $x_{3}=0.5$. Viscosity and porosity correspond to the case of water flowing in a mixture of calcarenite and sand: $\nu=0.01, \mu=10000$, and we set $\alpha=0.001$. The external forces on both domains 


\begin{tabular}{|c|c|c|c|c|c|c|c|c|c|c|c|c|}
\hline$h$ & $e\left(\boldsymbol{u}_{\mathrm{B}}\right)$ & $r\left(\boldsymbol{u}_{\mathrm{B}}\right)$ & $e\left(\boldsymbol{\omega}_{\mathrm{B}}\right)$ & $r\left(\boldsymbol{\omega}_{\mathrm{B}}\right)$ & $e\left(\boldsymbol{u}_{\mathrm{D}}\right)$ & $r\left(\boldsymbol{u}_{\mathrm{D}}\right)$ & $e\left(p_{\mathrm{B}}\right)$ & $r\left(p_{\mathrm{B}}\right)$ & $e\left(p_{\mathrm{D}}\right)$ & $r\left(p_{\mathrm{D}}\right)$ & $e(\lambda)$ & $r(\lambda)$ \\
\hline \multicolumn{13}{|c|}{ Fully mixed scheme (4.4) } \\
\hline 0.70711 & 1.02802 & - & 0.08636 & - & 0.65565 & - & 0.00404 & - & 0.64650 & - & 0.51608 & - \\
\hline 0.38079 & 0.66329 & 0.63216 & 0.04547 & 0.86511 & 0.30143 & 0.96758 & 0.00167 & 0.86588 & 0.24919 & 1.54026 & 0.37415 & 0.94712 \\
\hline 0.30610 & 0.45239 & 1.30206 & 0.03253 & 1.13929 & 0.21869 & 1.46952 & 0.00130 & 1.44871 & 0.12438 & 1.18266 & 0.22579 & 0.93668 \\
\hline 0.18503 & 0.29153 & 0.93254 & 0.02240 & 0.79149 & 0.16048 & 0.61483 & 0.00073 & 1.21498 & 0.05130 & 1.15932 & 0.17396 & 0.95387 \\
\hline 0.14412 & 0.18023 & 1.02275 & 0.01527 & 0.85417 & 0.10498 & 0.89264 & 0.00042 & 1.15692 & 0.02337 & 1.14603 & 0.10985 & 0.96014 \\
\hline 0.05487 & 0.11716 & 0.95707 & 0.00833 & 0.95944 & 0.05076 & 0.99002 & 0.00027 & 0.96289 & 0.01207 & 0.98594 & 0.05139 & 0.96765 \\
\hline 0.03564 & 0.07258 & 0.97681 & 0.00561 & 0.98670 & 0.03123 & 0.99197 & 0.00019 & 0.98441 & 0.00896 & 0.99455 & 0.02987 & 0.97732 \\
\hline \multicolumn{13}{|c|}{ Augmented mixed scheme (5.9) } \\
\hline 0.70711 & 1.02681 & - & 0.08574 & - & 0.65549 & - & 0.00416 & - & 0.64537 & - & 0.51899 & - \\
\hline 0.38079 & 0.62020 & 0.98418 & 0.04306 & 0.87429 & 0.26781 & 0.94902 & 0.00158 & 0.95434 & 0.24503 & 0.94234 & 0.38461 & 0.97729 \\
\hline 0.30610 & 0.42963 & 0.99011 & 0.02763 & 0.91368 & 0.17061 & 0.96547 & 0.00109 & 0.93939 & 0.10942 & 0.96471 & 0.21733 & 1.07908 \\
\hline 0.18503 & 0.27689 & 0.94556 & 0.01916 & 0.94842 & 0.12903 & 0.95084 & 0.00066 & 0.98741 & 0.04873 & 0.96933 & 0.16430 & 0.98544 \\
\hline 0.14412 & 0.16540 & 0.96134 & 0.01344 & 0.96083 & 0.08211 & 0.95171 & 0.00039 & 0.98177 & 0.01998 & 0.96297 & 0.08127 & 0.97476 \\
\hline 0.05487 & 0.10214 & 0.98608 & 0.00703 & 0.95798 & 0.04714 & 0.90989 & 0.00024 & 0.97506 & 0.00987 & 0.97250 & 0.04550 & 0.97732 \\
\hline 0.03564 & 0.06071 & 0.96110 & 0.00416 & 0.98465 & 0.02595 & 1.01103 & 0.00016 & 0.98411 & 0.00593 & 1.00141 & 0.02831 & 0.97460 \\
\hline \multicolumn{13}{|c|}{ Augmented mixed scheme (5.9) with vorticity space as in (5.12) } \\
\hline 0.70711 & 1.02643 & - & 0.06194 & - & 0.65421 & - & 0.00381 & - & 0.64501 & - & 0.49673 & - \\
\hline 0.38079 & 0.60941 & 0.96599 & 0.02469 & 1.12048 & 0.26554 & 0.95410 & 0.00155 & 0.95308 & 0.24394 & 0.95725 & 0.34426 & 1.02005 \\
\hline 0.30610 & 0.42550 & 0.98731 & 0.01822 & 1.51635 & 0.17022 & 0.92217 & 0.00102 & 0.94694 & 0.10922 & 0.96820 & 0.20871 & 0.97632 \\
\hline 0.18503 & 0.23411 & 1.01620 & 0.01014 & 1.85309 & 0.12850 & 0.98436 & 0.00061 & 0.93551 & 0.04855 & 0.95735 & 0.15809 & 0.99401 \\
\hline 0.14412 & 0.13869 & 0.98807 & 0.00607 & 1.92486 & 0.08173 & 0.96728 & 0.00039 & 0.93754 & 0.01980 & 0.96443 & 0.08067 & 0.95866 \\
\hline 0.05487 & 0.08619 & 0.97615 & 0.00413 & 1.93952 & 0.04684 & 0.93863 & 0.00022 & 0.98965 & 0.00982 & 0.94538 & 0.04491 & 0.97210 \\
\hline 0.03564 & 0.05175 & 0.99054 & 0.00279 & 1.97943 & 0.02433 & 0.98461 & 0.00013 & 1.90241 & 0.00563 & 1.10394 & 0.02635 & 0.96955 \\
\hline
\end{tabular}

Table 6.1: Example 1: Error history associated to fully mixed and augmented $\mathbb{R T}_{0}-\mathbb{N D}_{1}-\mathbb{R T}_{0}-\mathbf{P}_{0}-\mathbf{P}_{0}-\mathbf{P}_{1}$ discretizations (top and middle rows), and augmented $\mathbb{R T}_{0}-\mathbb{N D}_{2}-\mathbb{R T}_{0}-\mathbf{P}_{0}-\mathbf{P}_{0}-\mathbf{P}_{1} \mathrm{FE}$ family (bottom row) for problem (2.1)-(2.2) on a 3D domain.

correspond to gravity $\boldsymbol{f}_{\mathrm{D}}=\boldsymbol{f}_{\mathrm{B}}=(0,0,-0.98)^{\mathrm{t}}$, and a constant flowrate $\boldsymbol{u}_{\mathrm{D}} \cdot \boldsymbol{n}=(10,0,0)^{\mathrm{t}} \cdot \boldsymbol{n}$, is imposed on the right wall $\Gamma_{\mathrm{D}}^{\mathrm{in}}$, at $x_{1}=0$ (see sketch in figure 6.2 ), representing a subsurface flow in the $x_{1}$-direction. Normal Darcy velocities are set to zero everywhere else on $\Gamma_{\mathrm{D}}$. As in [9] we impose a smooth vorticity profile on the top of $\Gamma_{\mathrm{B}} \boldsymbol{\omega}_{\mathrm{B}} \times \boldsymbol{n}=\left(0,1 / 16-\left(x_{1}-1\right)^{2}, 0\right)^{\mathrm{t}} \times \boldsymbol{n}$, which takes into account the wind on the surface, and we also assume a compatible normal velocity on that same surface $\boldsymbol{u}_{\mathrm{B}} \cdot \boldsymbol{n}=\left(0,0,-x_{1} / 16+\left[\left(x_{1}-1\right)^{3}\right] / 3\right)^{\mathrm{t}} \cdot \boldsymbol{n}$. Everywhere else we set zero normal fluid velocity and zero tangential vorticity. A tetrahedral mesh with conforming interface is generated having 57426 vertices and 307544 elements, which in total correspond to 962639 degrees of freedom for $\mathbb{R T}_{0}-\mathbb{N D}_{1}-\mathbb{R T}_{0}-\mathbf{P}_{0}-\mathbf{P}_{0}-\mathbf{P}_{1}$ finite elements. Figure 6.2 depicts the domain configuration along with the approximate solutions, matching qualitatively the results from [9, 14].

\subsection{Perpendicular infiltration through a porous capsule}

In this test we present a model of coupled surface and subsurface flow where the top domain is the flow region and the bottom half of the domain represents e.g. a pellet, or a capsule. On the top left octant of $\Omega_{\mathrm{B}}$, denoted by $\Gamma_{\mathrm{B}}^{\mathrm{in}}$, we consider an inflow rate of $\boldsymbol{u}_{\mathrm{B}} \cdot \boldsymbol{n}=-0.01$ and on $\Gamma_{\mathrm{D}}^{\text {out }}$ (see the domain sketch in Figure 6.3) we set an outflow of fluid at rate $\boldsymbol{u}_{\mathrm{B}} \cdot \boldsymbol{n}=0.01$. Also on $\Gamma_{\mathrm{B}}^{\text {in }}$, we impose a smooth vorticity $\boldsymbol{\omega}_{\mathrm{B}} \times \boldsymbol{n}=\left(0,-0.01 x_{1} x_{2} x_{3}, 0\right)^{\mathrm{t}} \times \boldsymbol{n}$. On the remainder of $\partial \Omega$ we set zero normal velocities and tangential vorticity. As in the previous example, we take into account the gravity force acting on both domains $f_{\mathrm{D}}=\boldsymbol{f}_{\mathrm{B}}=(0,0,-0.98)^{\mathrm{t}}$, and employ the model parameters $\alpha=10, \nu=0.001, \mu=10000$. The mesh for $\Omega$ consists of 32768 vertices and 191452 tetrahedral elements representing 700835 degrees of freedom. As expected, from Figure 6.3 we observe flow patterns entering the domain through $\Gamma_{\mathrm{B}}^{\mathrm{in}}$, percolating through $\Sigma$, and leaving the domain through $\Gamma_{\mathrm{D}}^{\text {out }}$. These results have been obtained with the augmented mixed scheme (5.9). 

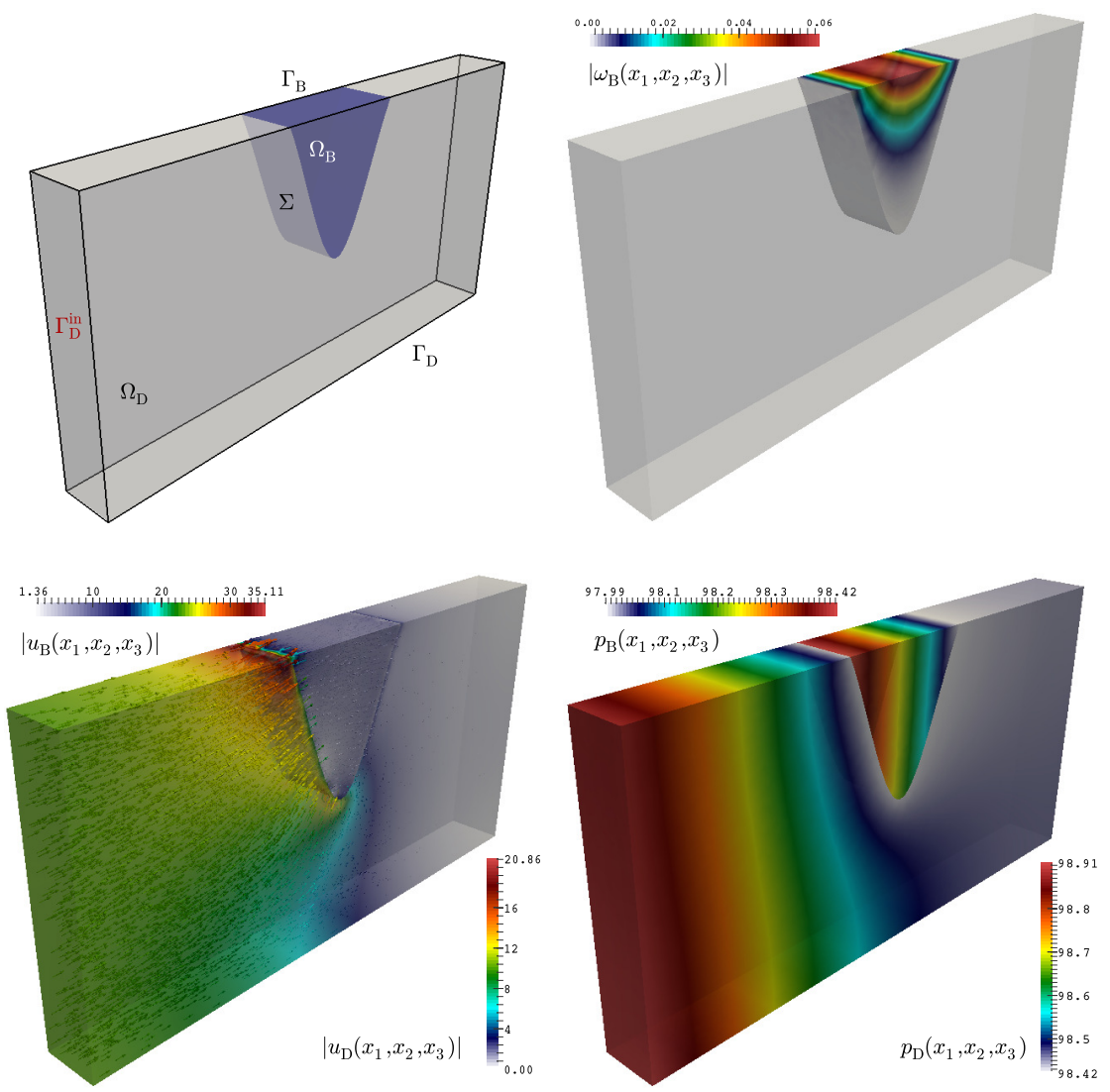

Figure 6.2: Example 2: Two-domain geometry and boundaries (top left), approximated Brinkman vorticity magnitude (top right), approximated velocity magnitude and vectors (bottom left), and computed pressure profiles (bottom right) for the Brinkman-Darcy coupling, using a fully-mixed scheme.
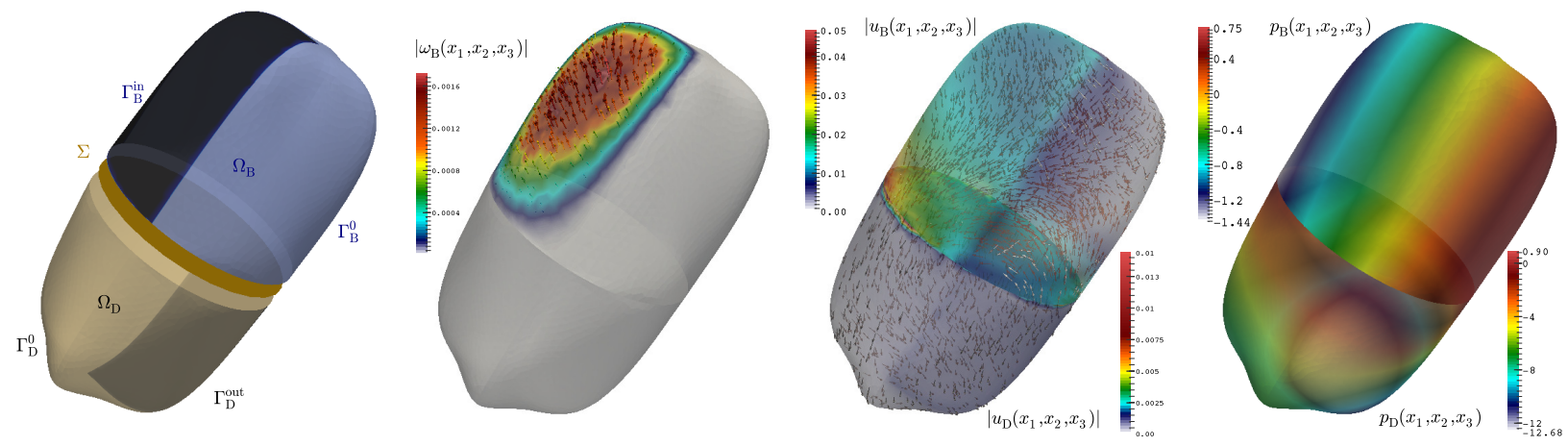

Figure 6.3: Example 3: From left to right: Two-domain geometry and boundaries, approximated Brinkman vorticity magnitude and vectors, approximated velocity magnitude and vectors, and computed pressure profile for the Brinkman-Darcy coupling, using an augmented finite element formulation.

\subsection{Flow simulations imposing Dirichlet conditions for the velocity}

Finally, we perform a test quite similar to Examples 2 and 3, but this time we impose Dirichlet conditions for the Brinkman and Darcy velocities on the external boundaries (which implies, in particular, that no boundary datum is required for the vorticity field), and employ an augmented formulation using a $\mathbb{R} \mathbb{T}_{0}-$ $\mathbb{N D}_{2}-\mathbb{R T}_{0}-\mathbf{P}_{0}-\mathbf{P}_{0}-\mathbf{P}_{1} \mathrm{FE}$ family. Now the Brinkman domain $\Omega_{\mathrm{B}}=[0,3] \times[0,0.2] \times[1,3 / 2]$ is on top of the Darcy domain $\Omega_{\mathrm{D}}=[0,3] \times[0,0.2] \times[0,1]$ (as in e.g. a two-layer subsurface flow). These 

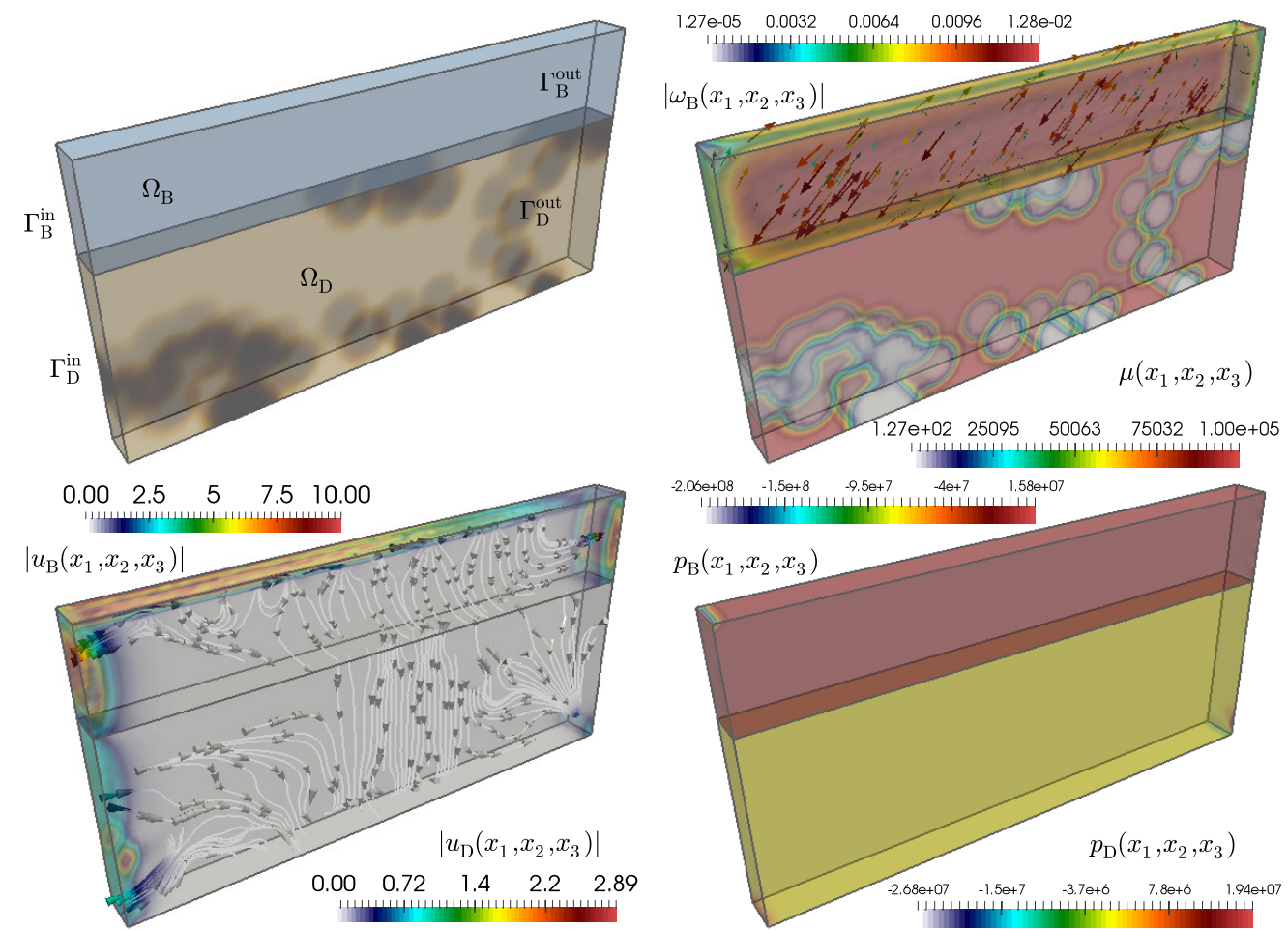

Figure 6.4: Example 4: Two-domain geometry and boundaries (top left), approximated Brinkman vorticity and Darcy permeability field (top right), approximated velocity (bottom left), and computed pressure profile (bottom right) for the BrinkmanDarcy coupling imposing Dirichlet boundary conditions for the velocity.

domains are discretized into structured tetrahedral meshes of 63195 and 94847 elements, respectively. The parameter $\mu$ (viscosity over permeability of the Darcy domain) is now highly heterogeneous (see top-left panel of Figure 6.4), and the remaining parameters take the values $\alpha=1 / 100, \nu=1, \kappa=2 \nu / \alpha$. We also assume the presence of a slight current along with gravity in the Brinkman domain, i.e. $\boldsymbol{f}_{\mathrm{B}}=(1,0,-0.98)^{\mathrm{t}}$. The boundary conditions for velocity are: $\boldsymbol{u}_{\mathrm{B}}=\left(100\left(x_{3}-1\right)\left(3 / 2-x_{3}\right), 0,0\right)^{\mathrm{t}}$ on $\Gamma_{\mathrm{B}}^{\mathrm{in}} \cup \Gamma_{\mathrm{B}}^{\text {out }}, \boldsymbol{u}_{\mathrm{B}}=(100(1-$ $\left.\left.x_{1}^{2} / 9\right), 0,0\right)^{\mathrm{t}}$ on the top surface, $\boldsymbol{u}_{\mathrm{D}}=(1,0,0)^{\mathrm{t}}$ on $\Gamma_{\mathrm{D}}^{\mathrm{in}}$, and on the sink at the lower part of $\Gamma_{\mathrm{D}}^{\text {out }}$, and no-slip Darcy velocity $\left(\boldsymbol{u}_{\mathrm{D}}=\mathbf{0}\right)$ on the bottom surface. No other conditions are imposed (e.g. on the front or back sides of the domain). Notice that Dirichlet conditions are implemented via a penalization strategy. We stress that even if this setting is not covered by our analysis, the obtained results (see the remaining panels in Figure 6.4) suggest that the augmented formulation proposed herein is capable to successfully handle problems involving Dirichlet velocity data. Nevertheless, the performance of the method in conditions where vorticity develops into more involved patterns, remains to be addressed.

\section{References}

[1] M. Ainsworth, J. Guzmán and F.-J. Sayas, Discrete extension operators for mixed finite element spaces on locally refined meshes. Math. Comp., in press. DOI:10.1090/mcom/3074

[2] A. Alonso and A. Valli, An optimal domain decomposition preconditioner for low-frequency time-harmonic Maxwell equations. Math. Comput. 68 (1999) 607-631.

[3] C. Amrouche, C. Bernardi, M. Dauge, and V. Girault, Vector potentials in tree-dimensional nonsmooth domains. Math. Methods. Appl. Sci. 21 (1998) 823-864.

[4] V. Anaya, G.N. Gatica, D. Mora, and R. Ruiz-Baier, An augmented velocity - vorticity - pressure formulation for the Brinkman equations. Int. J. Numer. Methods Fluids 79(3) (2015) 109-137.

[5] V. Anaya, D. Mora, R. Oyarzúa, and R. Ruiz-Baier, A priori and a posteriori error analysis of a mixed scheme for the Brinkman problem. Numer. Math., in press. DOI:10.1007/s00211-015-0758-x 
[6] T. Arbogast and D.S. Brunson, A computational method for approximating a Darcy-Stokes system governing a vuggy porous medium, Comput. Geosci. 11(3) (2007) 207-218.

[7] J.-L. Auriault, On the domain of validity of Brinkman's equation. Transp. Porous Med. 79 (2009) $215-223$.

[8] L. Badea, M. Discacciati, and A. Quarteroni, Numerical analysis of the Navier-Stokes/Darcy coupling, Numer. Math. 115 (2010) 195-227.

[9] C. Bernardi, F. Hecht, and F.Z. Nouri, A new finite-element discretization of the Stokes problem coupled with the Darcy equations. IMA J. Numer. Anal. 30 (2010) 61-93.

[10] C. Bernardi, F. Hecht, and O. Pironneau, Coupling Darcy and Stokes equations for porous media with cracks. ESAIM: Math. Model. Numer. Anal. 39(1) (2005) 7-35.

[11] A.-S. Bonnet-Ben Dhia, L. Chesnel, and P. Ciarlet, T-coercivity for scalar interface problems between dielectrics and metamaterials. ESAIM Math. Model. Numer. Anal. 46(6) (2012) 1363-1387.

[12] M. Braack and F. Schieweck, Equal-order finite elements with local projection stabilization for the DarcyBrinkman equations. Comput. Methods Appl. Mech. Engrg. 200(9-12) (2011) 1126-1136.

[13] F. Brezzi and M. Fortin, Mixed and Hybrid Finite Element Methods. Springer Verlag, New York, 1991.

[14] J. Camaño, G.N. Gatica, R. Oyarzúa, R. Ruiz-Baier, and P. Venegas, New fully-mixed finite element methods for the Stokes-Darcy coupling. Comput. Methods Appl. Mech. Engrg. 295 (2015) 362-395.

[15] L. Chesnel and P. Ciarlet, T-coercivity and continuous Galerkin methods: application to transmission problems with sign changing coefficients. Numer. Math., 124(1) (2013) 1-129.

[16] M. Discacciati and A. Quarteroni, Navier-Stokes/Darcy coupling: modeling, analysis, and numerical approximation. Rev. Mat. Complut. 22 (2009) 315-426.

[17] F. El Chami, G. Mansour, and T. Sayah, Error studies of the coupling Darcy-Stokes system with velocity-pressure formulation. Calcolo 49 (2012) 73-93.

[18] V.J. Ervin, E.W. Jenkins, and S. Sun, Coupled generalized nonlinear Stokes flow with flow through a porous medium, SIAM J. Numer. Anal. 47(2) (2009) 929-952.

[19] J. Galvis and M. Sarkis, Non-matching mortar discretization analysis for the coupling Stokes-Darcy equations. Electr. Trans. Numer. Anal. 26 (2007) 350-384.

[20] G.N. Gatica, A Simple Introduction to the Mixed Finite Element Method. Theory and Applications. SpringerVerlag, Berlin, 2014.

[21] G.N. Gatica, G.C. Hsiao, and S. Meddahi, A coupled mixed finite element method for the interaction problem between and electromagnetic field and elastic body. SIAM J. Numer. Anal. 48(4) (2010) 1338-1368.

[22] G.N. Gatica, A. Márquez, and S. Meddahi, Analysis of the coupling of primal and dual-mixed finite element methods for a two-dimensional fluid-solid interaction problem. SIAM J. Numer. Anal. 45(5) (2007) $2072-2097$.

[23] G.N. Gatica, A. Márquez, and S. Meddahi, Analysis of the coupling of Lagrange and Arnold-Falk-Winther finite elements for a fluid-solid interaction problem in 3D. SIAM J. Numer. Anal. 50(3) (2012) 1648-1674.

[24] G.N. Gatica, R. Oyarzúa, and F.-J. Sayas, Analysis of fully-mixed finite element methods for the Stokes-Darcy coupled problem. Math. Comput. 80(276) (2011) 1911-1948.

[25] G.N. Gatica, R. Oyarzúa, and F.-J. Sayas, A twofold saddle point approach for the coupling of fluid flow with nonlinear porous media flow. IMA J. Numer. Anal. 32(3) (2012) 845-887.

[26] G.N. Gatica, R. Ruiz-Baier, and G. Tierra, A mixed finite element method for the Darcy equations with pressure-dependent porosity. Math. Comp. 85(297) (2016) 1-33.

[27] V. Girault and P. A. Raviart, Finite element methods for Navier-Stokes equations. Theory and algorithms. Springer-Verlag, Berlin, 1986.

[28] P. Grisvart, Singularities in Boundary Value Problems. Recherches en Mathématiques Appliquées. Springer, Berlin, vol. 22, 1992. 
[29] M. Lesinigo, C. D'Angelo, and A. Quarteroni, A multiscale Darcy-Brinkman model for fluid flow in fractured porous media. Numer. Math. 117 (2011) 717-752.

[30] W. McLean, Strongly Elliptic Systems and Boundary Integral Equations. Cambridge University Press, Cambridge, 2000.

[31] P. Monk, Finite Element Methods for Maxwell's Equations. Oxford University Press, New York, 2003.

[32] B. Rivière, Analysis of a discontinuous finite element method for the coupled Stokes and Darcy problems. J. Sci. Comput. 22(1) (2005) 479-500. 